\title{
The Projected Dark and Baryonic Ellipsoidal Structure of 20 CLASH Galaxy Clusters*
}

\author{
Keiichi Umetsu ${ }^{1}\left(\mathbb{D}\right.$, Mauro Sereno ${ }^{2,3}$, Sut-Ieng Tam ${ }^{4}$, I-Non Chiu ${ }^{1}$, Zuhui Fan ${ }^{5,6}$, Stefano Ettori ${ }^{2,7}$, Daniel Gruen ${ }^{8,9,28}$, \\ Teppei Okumura $^{1}$, Elinor Medezinski ${ }^{10}$, Megan Donahue ${ }^{11}$, Massimo Meneghetti ${ }^{12}$ (i) , Brenda Frye ${ }^{13}$, Anton Koekemoer ${ }^{14}$, \\ Tom Broadhurst ${ }^{15,16}$, Adi Zitrin ${ }^{17}$ (i), Italo Balestra ${ }^{18,19}$ (i) , Narciso Benítez ${ }^{20}$, Yuichi Higuchi ${ }^{1}$, Peter Melchior $^{10}$, \\ Amata Mercurio $^{21}$, Julian Merten ${ }^{22}$, Alberto Molino ${ }^{23}$, Mario Nonino ${ }^{19}$, Marc Postman ${ }^{14}$, Piero Rosati ${ }^{24}$, Jack Sayers ${ }^{25}$ (i), and \\ Stella Seitz ${ }^{26,27}$ \\ ${ }^{1}$ Institute of Astronomy and Astrophysics, Academia Sinica, P.O. Box 23-141, Taipei 10617, Taiwan; keiichi@asiaa.sinica.edu.tw \\ ${ }^{2}$ INAF-Osservatorio di Astrofisica e Scienza dello Spazio di Bologna, via Piero Gobetti 93/3, I-40129 Bologna, Italy \\ 3 Dipartimento di Fisica e Astronomia, Università di Bologna, via Piero Gobetti 93/2, I-40129 Bologna, Italy \\ ${ }^{4}$ Centre for Extragalactic Astronomy, Department of Physics, Durham University, Durham DH1 3LE, UK \\ ${ }^{5}$ South-Western Institute for Astronomy Research, Yunnan University, Kunming 650500, People's Republic of China \\ ${ }^{6}$ Department of Astronomy, School of Physics, Peking University, Beijing 100871, People's Republic of China \\ INFN, Sezione di Bologna, viale Berti Pichat 6/2, I-40127 Bologna, Italia \\ ${ }^{8}$ SLAC National Accelerator Laboratory, Menlo Park, CA 94025, USA \\ ${ }^{9}$ KIPAC, Physics Department, Stanford University, Stanford, CA 94305, USA \\ ${ }^{10}$ Department of Astrophysical Sciences, 4 Ivy Lane, Princeton, NJ 08544, USA \\ ${ }^{11}$ Department of Physics and Astronomy, Michigan State University, East Lansing, MI 48824, USA \\ ${ }^{12}$ INAF-Osservatorio Astronomico di Bologna, via Ranzani 1, I-40127 Bologna, Italy \\ ${ }^{13}$ University of Arizona, 933 N. Cherry Ave, Tucson, AZ 85721, USA \\ ${ }^{14}$ Space Telescope Science Institute, 3700 San Martin Drive, Baltimore, MD 21218, USA \\ ${ }^{15}$ Department of Theoretical Physics, University of Basque Country UPV/EHU, E-48080 Bilbao, Spain \\ ${ }^{16}$ IKERBASQUE, Basque Foundation for Science, E-48013 Bilbao, Spain \\ ${ }^{17}$ Physics Department, Ben-Gurion University of the Negev, P.O. Box 653, Be'er-Sheva 8410501, Israel \\ ${ }_{18}$ University Observatory Munich, Scheinerstrasse 1, D-81679 Munich, Germany \\ ${ }^{19}$ INAF-Osservatorio Astronomico di Trieste, Via Tiepolo 11, I-34131 Trieste, Italy
${ }^{20}$ Instituto de Astrofisica de Andalucia (CSIC), Glorieta de la Astronomìa s/n, Granada, E-18008, Spain \\ ${ }^{21}$ INAF-Osservatorio Astronomico di Capodimonte, via Moiariello 16, I-80131 Napoli, Italy \\ ${ }^{22}$ Oxford University, Keble Road, Oxford OX1 3RH, UK \\ ${ }^{23}$ Universidade de São Paulo, Instituto de Astronomia, Geofísica e Ciências Atmosféricas, Rua do Matão 1226, 05508-090, São Paulo, Brazil \\ ${ }^{24}$ Università di Ferrara, Via Saragat 1, I-44122 Ferrara, Italy \\ ${ }^{25}$ Division of Physics, Math, and Astronomy, California Institute of Technology, Pasadena, CA 91125, USA \\ ${ }^{26}$ Universitäts-Sternwarte, München, Scheinerstr. 1, D-81679 München, Germany \\ ${ }^{27}$ Max-Planck-Institut für extraterrestrische Physik, Giessenbachstr. 1, D-85748 Garching \\ Received 2018 March 30; revised 2018 May 3; accepted 2018 May 8; published 2018 June 18
}

\begin{abstract}
We reconstruct the two-dimensional (2D) matter distributions in 20 high-mass galaxy clusters selected from the CLASH survey by using the new approach of performing a joint weak gravitational lensing analysis of 2D shear and azimuthally averaged magnification measurements. This combination allows for a complete analysis of the field, effectively breaking the mass-sheet degeneracy. In a Bayesian framework, we simultaneously constrain the mass profile and morphology of each individual cluster, assuming an elliptical Navarro-Frenk-White halo characterized by the mass, concentration, projected axis ratio, and position angle (PA) of the projected major axis. We find that spherical mass estimates of the clusters from azimuthally averaged weak-lensing measurements in previous work are in excellent agreement with our results from a full 2D analysis. Combining all 20 clusters in our sample, we detect the elliptical shape of weak-lensing halos at the $5 \sigma$ significance level within a scale of $2 \mathrm{Mpc} h^{-1}$. The median projected axis ratio is $0.67 \pm 0.07$ at a virial mass of $M_{\text {vir }}=(15.2 \pm 2.8) \times 10^{14} M_{\odot}$, which is in agreement with theoretical predictions from recent numerical simulations of the standard collisionless cold dark matter model. We also study misalignment statistics of the brightest cluster galaxy, X-ray, thermal Sunyaev-Zel'dovich effect, and strong-lensing morphologies with respect to the weak-lensing signal. Among the three baryonic tracers studied here, we find that the X-ray morphology is best aligned with the weak-lensing mass distribution, with a median misalignment angle of $|\Delta \mathrm{PA}|=21^{\circ} \pm 7^{\circ}$. We also conduct a stacked quadrupole shear analysis of the 20 clusters assuming that the X-ray major axis is aligned with that of the projected mass distribution. This yields a consistent axis ratio of $0.67 \pm 0.10$, suggesting again a tight alignment between the intracluster gas and dark matter.
\end{abstract}

Key words: cosmology: observations - dark matter - galaxies: clusters: general - galaxies: clusters: intracluster medium - gravitational lensing: weak

\footnotetext{
* Based in part on data collected at the Subaru Telescope, which is operated by the National Astronomical Society of Japan.

${ }^{28}$ Einstein Fellow.
}

\section{Introduction}

Galaxy clusters, as the most massive objects formed in the universe, represent fundamental probes of cosmology. Clusters contain rich information about the initial conditions for structure formation, the emergence of large-scale structure 
over cosmic time, and the properties of dark matter (DM). In the standard picture of hierarchical structure formation, determining the abundance of rare massive clusters above a given mass provides a powerful test of growth of structure (e.g., Bahcall \& Fan 1998) because cluster-scale halos populate the exponential tail of the cosmic mass function. Statistical properties of clusters can thus be used to yield unique constraints on cosmological parameters and models of cosmic structure formation (Allen et al. 2004; Vikhlinin et al. 2009; Mantz et al. 2010), complementing standard cosmological probes, such as cosmic microwave background (CMB) anisotropy, large-scale galaxy clustering, distant supernova, and cosmic shear observations.

The most critical ingredient for cluster-based tests of structure formation is the distribution and amount of DM in cluster halos. In this context, the standard $\Lambda$ cold dark matter $(\Lambda \mathrm{CDM})$ model and its variants, such as self-interacting DM (SIDM; Spergel \& Steinhardt 2000) and wave DM ( $\psi$ DM; Schive et al. 2014), provide a range of observationally testable predictions. $N$-body simulations in the standard $\Lambda C D M$ model reveal that clusters form through successive mergers of groups and smaller clusters, as well as through the smooth accretion of matter from the surrounding filamentary structure (Colberg et al. 2000; Shaw et al. 2006), leading to a highly anisotropic geometry in which infall and merging of matter tend to take place along preferential directions. The process results in the emergence of the filamentary cosmic web, as observed in galaxy redshift surveys (Colless et al. 2001; Tegmark et al. 2004; Geller et al. 2011). Cluster halos are formed in overdense regions where the filaments intersect. The shape of halos is generally triaxial with a preference for prolate shapes (Jing \& Suto 2002; Shaw et al. 2006), reflecting the collisionless nature of DM (Ostriker \& Steinhardt 2003). On average, older halos are more relaxed and are thus more spherical. Since more massive halos form later on average, clusters are thus expected to be more elongated than less massive systems (Despali et al. 2014). Accretion of matter from the surrounding large-scale environment also plays a key role in determining the shape and orientation of cluster halos. The halo orientation tends to be in the preferential infall direction of the subhalos and hence aligned along the surrounding filaments (Shaw et al. 2006). The shape and orientation of galaxy clusters thus provide an independent test of models of structure formation.

Gravitational lensing offers a direct probe of the cluster mass distribution through observations of weak shear lensing (e.g., Gruen et al. 2014; von der Linden et al. 2014; Hoekstra et al. 2015; Melchior et al. 2017; Sereno et al. 2017a; Medezinski et al. 2018), weak magnification lensing (e.g., Hildebrandt et al. 2011; Umetsu et al. 2011b; Coupon et al. 2013; Chiu et al. 2016; Tudorica et al. 2017), strong gravitational lensing (e.g., Broadhurst et al. 2005a; Zitrin et al. 2013; Jauzac et al. 2015; Cerny et al. 2017; Diego et al. 2018), and the combination of these effects (e.g., Umetsu et al. 2011a; Coe et al. 2012; Medezinski et al. 2013; Umetsu et al. 2015, 2016). The critical advantage of gravitational lensing is its unique ability to map the mass distribution independently of assumptions about their physical or dynamical state. Cluster lensing thus provides a direct and powerful way to test predictions of halo density structure dominated by DM.

Cluster lensing observations have established that the projected total mass distribution within individual and ensemble-averaged clusters can be well described by sharply steepening density profiles (Umetsu et al. 2011a, 2014, 2016; Newman et al. 2013; Okabe et al. 2013; Okabe \& Smith 2016), with a near-universal shape (Niikura et al. 2015; Umetsu \& Diemer 2017), as predicted for halos dominated by collisionless DM in quasi-gravitational equilibrium (e.g., Navarro et al. 1996, 1997; Hjorth \& Williams 2010; Williams \& Hjorth 2010). Subsequent cluster lensing studies targeting lensing-unbiased samples (Du et al. 2015; Merten et al. 2015; Okabe \& Smith 2016; Umetsu et al. 2016; Cibirka et al. 2017) have shown that the degree of halo concentration derived for these clusters agrees well with theoretical models that are calibrated for recent $\Lambda \mathrm{CDM}$ cosmologies with a relatively high normalization (Bhattacharya et al. 2013; Dutton \& Macciò 2014; Meneghetti et al. 2014; Diemer \& Kravtsov 2015). These results are in support of the standard explanation for DM as effectively collisionless and non-relativistic on sub-Mpc scales and beyond, with an excellent match between cluster lensing data and $\Lambda C D M$ predictions.

The CLUster Multi-Probes in Three Dimensions (CLUMP3D; Sereno et al. 2017b) program aims to study intrinsic threedimensional (3D) properties of high-mass galaxy clusters and to test models of cluster formation. By exploiting rich data sets ranging from the X-ray, through optical, to radio wavelengths, we can constrain the 3D geometry and internal structure of individual clusters, together with the equilibrium status of the intracluster gas residing in cluster DM halos (e.g., Morandi et al. 2012; Sereno et al. 2013). In the approach developed by Sereno et al. (2013), we exploit the combination of X-ray and thermal Sunyaev-Zel'dovich effect (SZE) observations to constrain the line-of-sight elongation of the intracluster gas in a parametric triaxial framework. Employing minimal geometric assumptions about the matter and gas distributions, we then couple the constraints from gravitational lensing, X-ray, and SZE data sets in a Bayesian inference framework (Sereno et al. 2013; Umetsu et al. 2015; Sereno et al. 2017b). This multiprobe method allows constraints on the intrinsic shape and orientation of the matter and gas distributions to be improved without assuming hydrostatic equilibrium.

As part of the CLUMP-3D program, we present in this paper a two-dimensional (2D) weak-lensing analysis of wide-field shear and magnification data for a sample of 20 high-mass clusters, for which high-quality multiwavelength data sets are available from the CLASH survey (Postman et al. 2012) and dedicated follow-up programs (Donahue et al. 2014; Rosati et al. 2014; Umetsu et al. 2014, 2016; Czakon et al. 2015; Merten et al. 2015; Zitrin et al. 2015). In this work, we analyze the ground-based weak-lensing data of Umetsu et al. (2014) obtained from deep multiband imaging taken primarily with the Suprime-Cam on the Subaru Telescope $\left(34^{\prime} \times 27^{\prime}\right.$; Miyazaki et al. 2002). For our southernmost cluster (RX J2248-4431), which is not observable from Subaru, we analyze data obtained with the Wide-Field Imager (WFI) at the ESO $2.2 \mathrm{~m} \mathrm{MPG/}$ ESO telescope at La Silla (Gruen et al. 2013; Umetsu et al. 2014).

The primary aim of this paper is to perform an unbiased mass reconstruction in our 20 cluster fields, from which to simultaneously constrain the structure and morphology of the cluster mass distribution, both individually and statistically. This allows us to compare the position angles of cluster major axes determined from our wide-field weak-lensing analysis to those of baryonic tracers and central lensing maps inferred by 
Donahue et al. (2015, 2016). This work has two companion papers: the triaxial modeling and ensemble characterization of the 20 CLASH clusters by Chiu et al. (2018) from a joint analysis of weak- and strong-lensing data sets and the multiprobe triaxial modeling of 16 CLASH X-ray-selected clusters by Sereno et al. (2018) from a joint analysis of weaklensing, strong-lensing, X-ray, and SZE data sets.

This paper is organized as follows. In Section 2, we describe the basic theory of weak gravitational lensing by galaxy clusters. After summarizing the properties of the cluster sample and the observational data, we outline in Section 3 the formalism and procedure for reconstructing the cluster mass distribution from a 2D weak-lensing analysis of wide-field shear and magnification data. In Section 4, we present the results of 2D mass modeling of weak-lensing maps for our sample. Section 5 is devoted to the discussion of the results. Finally, a summary is given in Section 6.

Throughout this paper, we assume a spatially flat $\Lambda \mathrm{CDM}$ cosmology with $\Omega_{\mathrm{m}}=0.27, \Omega_{\Lambda}=0.73$, and a Hubble constant of $H_{0}=100 h \mathrm{~km} \mathrm{~s}^{-1} \mathrm{Mpc}^{-1}=70 h_{70} \mathrm{~km} \mathrm{~s}^{-1} \mathrm{Mpc}^{-1}$ with $h=$ $0.7 h_{70}=0.7$. We denote the mean matter density of the universe as $\rho_{\mathrm{m}}$ and the critical density of the universe as $\rho_{\mathrm{c}}$. We adopt the standard notation $M_{\Delta \mathrm{c}}$ or $M_{\Delta \mathrm{m}}$ to denote the mass enclosed within a sphere of radius $r_{\Delta \mathrm{c}}$ or $r_{\Delta \mathrm{m}}$, within which the mean overdensity equals $\Delta_{\mathrm{c}} \times \rho_{\mathrm{c}}(z)$ or $\Delta_{\mathrm{m}} \times \rho_{\mathrm{m}}(z)$ at a particular redshift $z$, such that $M_{\Delta \mathrm{c}}=(4 \pi / 3) \Delta_{\mathrm{c}} \rho_{\mathrm{c}}(z) r_{\Delta \mathrm{c}}^{3}$ and $M_{\Delta \mathrm{m}}=$ $(4 \pi / 3) \Delta_{\mathrm{m}} \rho_{\mathrm{m}}(z) r_{\Delta \mathrm{m}}^{3}$. We compute the virial mass and radius, $M_{\mathrm{vir}}$ and $r_{\mathrm{vir}}$, using an expression for $\Delta_{\mathrm{vir}}(z)$ based on the spherical collapse model (Appendix A of Kitayama et al. 1998). For a given overdensity $\Delta$, the concentration parameter is defined as $c_{\Delta}=r_{\Delta} / r_{\mathrm{s}}$. All quoted errors are $1 \sigma$ confidence limits unless otherwise stated.

\section{Basics of Cluster Gravitational Lensing}

\subsection{Shear and Magnification}

The effect of weak gravitational lensing on background sources is characterized by the convergence, $\kappa$, and the shear with spin 2 rotational symmetry, $\gamma=|\gamma| e^{2 i \phi}$ (e.g., Bartelmann \& Schneider 2001; Umetsu 2010). The convergence causes an isotropic magnification due to lensing and is defined as the surface mass density $\Sigma$ in units of the critical surface density for lensing, $\kappa=\Sigma / \Sigma_{\mathrm{c}}$, where

$$
\Sigma_{\mathrm{c}}=\frac{c^{2} D_{\mathrm{s}}}{4 \pi G D_{1} D_{\mathrm{ls}}} \equiv \frac{c^{2}}{4 \pi G D_{1}} \beta^{-1}
$$

with $c$ the speed of light, $G$ the gravitational constant, and $D_{1}$, $D_{\mathrm{s}}$, and $D_{\mathrm{ls}}$ the observer-lens, observer-source, and lenssource angular diameter distances, respectively. The dimensionless factor $\beta\left(z, z_{1}\right)=D_{\mathrm{ls}} / D_{\mathrm{s}}$ describes the geometric lensing strength as a function of source redshift $z$ and lens redshift $z_{1}$, where $\beta\left(z, z_{1}\right)=0$ for unlensed objects with $z \leqslant z_{1}$. Hence, the shear and convergence depend on the source and lens redshifts $\left(z, z_{1}\right)$, as well as on the image position $\boldsymbol{\theta}$.

The gravitational shear $\gamma$ is directly observable from image ellipticities of background galaxies in the weak regime, $\kappa \ll 1$. The shear and convergence fields are related by (Kaiser \& Squires 1993)

$$
\gamma(\boldsymbol{\theta})=\int d^{2} \theta^{\prime} D\left(\boldsymbol{\theta}-\boldsymbol{\theta}^{\prime}\right) \kappa\left(\boldsymbol{\theta}^{\prime}\right),
$$

with $D(\boldsymbol{\theta})$ the complex kernel $D(\boldsymbol{\theta})=\left(\theta_{2}^{2}-\theta_{1}^{2}-2 i \theta_{1}\right.$ $\left.\theta_{2}\right) /\left(\pi|\boldsymbol{\theta}|^{4}\right)$. In general, the observable quantity for weak lensing is not $\gamma$ but the complex reduced shear,

$$
g=\frac{\gamma}{1-\kappa},
$$

which remains invariant under the global transformation $\kappa$ $(\boldsymbol{\theta}) \rightarrow \lambda \kappa(\boldsymbol{\theta})+1-\lambda$ and $\gamma(\boldsymbol{\theta}) \rightarrow \lambda \gamma(\boldsymbol{\theta})$ with an arbitrary constant $\lambda \neq 0$ (for a fixed source redshift $z$ ). This is known as the mass-sheet degeneracy (Schneider \& Seitz 1995). In principle, this degeneracy can be broken or alleviated, for example, by measuring the magnification factor $\mu$ in the subcritical regime,

$$
\mu=\frac{1}{(1-\kappa)^{2}-|\gamma|^{2}} \equiv \frac{1}{\Delta_{\mu}},
$$

which transforms as $\mu(\boldsymbol{\theta}) \rightarrow \lambda^{-2} \mu(\boldsymbol{\theta})$. For simplicity of notation, we often use the inverse magnification $\Delta_{\mu}=\mu^{-1}$ rather than the magnification.

\subsection{Source Redshift Distribution}

For statistical weak-lensing measurements, we consider a population of source galaxies characterized by their mean redshift distribution function, $\bar{N}(z)$. In general, we use different size, magnitude, color, and quality cuts in background selection for measuring shear and magnification, which results in different $\bar{N}(z)$. In contrast to the former analysis, the latter does not require background sources to be spatially resolved, while it does require a stringent flux limit against incompleteness effects (Umetsu et al. 2014; Chiu et al. 2016). The sourceaveraged lensing depth for a given population $(X=g, \mu)$ is

$$
\langle\beta\rangle_{X}=\left[\int_{0}^{\infty} d z \bar{N}_{X}(z) \beta(z)\right]\left[\int_{0}^{\infty} d z \bar{N}_{X}(z)\right]^{-1} .
$$

Let us introduce the relative lensing strength of a given source population as $\langle W\rangle_{X}=\langle\beta\rangle_{X} / \beta_{\infty}$, with $\beta_{\infty} \equiv \beta\left(z \rightarrow \infty, z_{1}\right)$ defined relative to a fiducial source in the far background (Bartelmann \& Schneider 2001). The associated critical density is $\Sigma_{\mathrm{c}, \infty}\left(z_{1}\right)=c^{2} /\left(4 \pi G D_{1}\right) \beta_{\infty}^{-1}$. Hereafter, we use the farbackground fields $\kappa_{\infty}(\boldsymbol{\theta})$ and $\gamma_{\infty}(\boldsymbol{\theta})$ to describe the projected mass distribution.

\subsection{Pixelized Mass Distribution}

We pixelize the convergence field, $\kappa_{\infty}(\boldsymbol{\theta})=\Sigma_{\mathrm{c}, \infty}^{-1} \Sigma(\boldsymbol{\theta})$, into a regular grid of pixels, and describe $\kappa_{\infty}(\boldsymbol{\theta})$ by a linear combination of basis functions $B\left(\boldsymbol{\theta}-\boldsymbol{\theta}^{\prime}\right)$,

$$
\kappa_{\infty}(\boldsymbol{\theta})=\Sigma_{\mathrm{c}, \infty}^{-1} \sum_{n=1}^{N_{\mathrm{pix}}} B\left(\boldsymbol{\theta}-\boldsymbol{\theta}_{n}\right) \Sigma_{n} .
$$

To avoid the loss of information due to oversmoothing, we take the basis function to be the Dirac delta function, $B\left(\boldsymbol{\theta}-\boldsymbol{\theta}^{\prime}\right)=(\Delta \theta)^{2} \delta_{\mathrm{D}}^{2}\left(\boldsymbol{\theta}-\boldsymbol{\theta}^{\prime}\right)$, with $\Delta \theta$ a constant grid spacing (Umetsu et al. 2015). Our model (signal) is specified by a vector of parameters containing cell-averaged surface mass densities (Umetsu et al. 2015),

$$
\boldsymbol{s}=\left\{\Sigma_{n}\right\}_{n=1}^{N_{\text {pix }}} .
$$




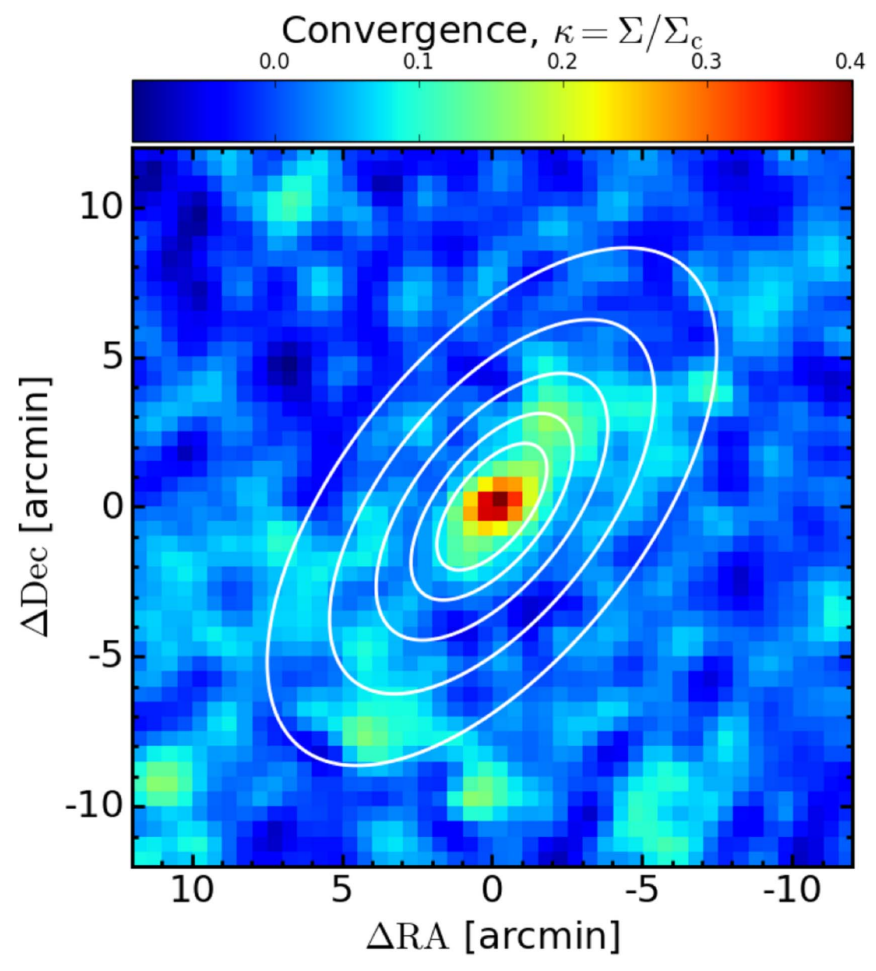

Figure 1. Example of our weak-lensing mass reconstruction shown for the cluster MACS J0329.7-0211 at $z=0.45$. The mass map is $24^{\prime} \times 24^{\prime}$ in size (5.9 $h^{-1}$ proper Mpc on a side) and centered on the BCG. The color bar indicates the lensing convergence $\kappa(\boldsymbol{\theta})=\left\langle\Sigma_{\mathrm{c}}^{-1}\right\rangle \Sigma(\boldsymbol{\theta})$, scaled to the mean depth of Subaru weak-lensing observations, $1 /\left\langle\Sigma_{\mathrm{c}}^{-1}\right\rangle=3.65 \times 10^{15} h M_{\odot}$ $\mathrm{Mpc}^{-2}$. For visualization purposes, the mass map is smoothed with a $1 ! 2$ FWHM Gaussian. North is to the top, east to the left. Elliptical isodensity contours of the best-fit elliptical Navarro-Frenk-White model are shown in white. The contour levels are logarithmically spaced from $\kappa=0.01$ to $\kappa=0.1$.

The complex shear field is expressed as

$$
\gamma_{\infty}(\boldsymbol{\theta})=\Sigma_{\mathrm{c}, \infty}^{-1} \sum_{n=1}^{N_{\mathrm{pix}}} \mathcal{D}\left(\boldsymbol{\theta}-\boldsymbol{\theta}_{n}\right) \Sigma_{n},
$$

with $\mathcal{D} \equiv D \otimes B=\pi^{-1}(\Delta \theta)^{2} D$ an effective complex kernel (see Equation (2)). Hence, both $\kappa_{\infty}(\boldsymbol{\theta})$ and $\gamma_{\infty}(\boldsymbol{\theta})$ can be written as linear combinations of mass coefficients.

It is important to note that, because of the choice of the basis function, an unbiased extraction of the mass coefficients $\left\{\Sigma_{n}\right\}_{n=1}^{N_{\text {pix }}}$ is possible by performing a spatial integral of $\kappa_{\infty}(\boldsymbol{\theta})$ over a certain area. Such operations include smoothing (Figure 1), azimuthal averaging for a mass profile measurement (Umetsu et al. 2015), and fitting with smooth parametric functions (Section 4).

\section{Weak-lensing Data and Methodology}

The present work is a full 2D generalization of the weaklensing study by Umetsu et al. (2014), who conducted a onedimensional (1D) weak-lensing analysis of azimuthally averaged shear and magnification measurements for a sample of 20 CLASH clusters (Section 3.1). A practical limitation of a shear-only analysis is the inherent mass-sheet degeneracy (Falco et al. 1985; Gorenstein et al. 1988; Schneider \& Seitz 1995; Seitz \& Schneider 1997; Bradač et al. 2004). One can substantially alleviate this degeneracy by using the complementary combination of shear and magnification
(Schneider et al. 2000; Umetsu \& Broadhurst 2008; Rozo and Schmidt 2010; Umetsu 2013). Measuring the two complementary effects also allows the internal consistency of weaklensing measurements (Umetsu et al. 2014) to be tested. Besides, in the context of the CLUMP-3D program, obtaining accurate mass maps has the important advantage of being able to identify local mass structures and to directly compare them with multiwavelength observations.

In this study, we use the ground-based weak-lensing data obtained by the CLASH collaboration (Section 3.2). Our shear catalogs (Section 3.3) as well as azimuthally averaged magnification profiles (Section 3.4) have already been published in Umetsu et al. (2014). Data products from the CLASH survey, including the reduced Subaru images, weight maps, and multiband photometric catalogs, are publicly available at the Mikulski Archive for Space Telescopes (MAST). ${ }^{29}$ Details of the image reduction, photometry, background source selection, and the creation of our weak-lensing shear catalogs are given in Umetsu et al. (2014, see their Section 4). More details on weak-lensing systematics are presented in Section 3 of Umetsu et al. (2016). Thus, we provide here only a summary of the procedures. Section 3.6 summarizes the major differences between our analysis and that of Umetsu et al. (2014) and Umetsu et al. (2016).

\subsection{Cluster Sample}

The cluster sample studied in the CLUMP-3D program (Sereno et al. 2017b; Chiu et al. 2018; Sereno et al. 2018) stems from the wide-field weak-lensing analysis of Umetsu et al. (2014). The sample comprises two subsamples, one with 16 $\mathrm{X}$-ray-selected clusters and another with four high-magnification clusters (Table 1). Both subsamples were taken from the CLASH survey (Postman et al. 2012), a 524 orbit Hubble Space Telescope (HST) Multi-Cycle Treasury program targeting 25 high-mass clusters.

Here, 20 CLASH clusters were selected to have X-ray temperatures above $5 \mathrm{keV}$ and to have a high degree of regularity in their X-ray morphology. Specifically, these clusters show well-defined central surface brightness peaks and nearly concentric isophotes in Chandra X-ray images (Postman et al. 2012). The CLASH X-ray criteria ensure welldefined cluster centers, reducing the effects of cluster miscentering (see below). Meneghetti et al. (2014) characterized intrinsic and observational properties of the CLASH $\mathrm{X}$-ray-selected subsample by analyzing simulated halos chosen to match these individual clusters in terms of the X-ray morphological regularity. Their simulations suggest that this subsample is largely free of orientation bias and dominantly composed of relaxed clusters $(\sim 70 \%)$, but it also contains a non-negligible fraction $(\sim 30 \%)$ of unrelaxed clusters. Another subset of five clusters was selected for their high-lensing magnification. These clusters often turn out to be dynamically disturbed, complex merging systems (e.g., Medezinski et al. 2013; Zitrin et al. 2013; Balestra et al. 2016; Jauzac et al. 2017). A complete definition of the CLASH sample is given in Postman et al. (2012).

Donahue et al. (2016) presented uniformly estimated X-ray morphological statistics for the full sample of $25 \mathrm{CLASH}$ clusters using Chandra X-ray observations. Comparing the $\mathrm{X}$-ray morphological properties between the two CLASH

\footnotetext{
${ }^{29}$ https://archive.stsci.edu/prepds/clash/
} 
Table 1

Cluster Sample and Elliptical Navarro-Frenk-White Model Parameters

\begin{tabular}{|c|c|c|c|c|c|c|c|c|}
\hline Cluster & Redshift $^{\mathrm{a}}$ & $\begin{array}{c}\text { R.A. }^{\text {b }} \\
(\mathrm{J} 2000.0)\end{array}$ & $\begin{array}{c}\text { Decl. }^{\mathrm{b}} \\
(\mathrm{J} 2000.0)\end{array}$ & $\begin{array}{c}M_{200 c} \\
\left(10^{14} M_{\odot} h_{70}^{-1}\right)\end{array}$ & $c_{200 c}$ & $q_{\perp}{ }^{\mathrm{c}}$ & $\begin{array}{c}\mathrm{PA}^{\mathrm{d}} \\
\text { (degrees) }\end{array}$ & $\chi^{2} /$ dof $^{e}$ \\
\hline \multicolumn{9}{|l|}{ X-ray Selected: } \\
\hline Abell 383 & 0.187 & 02:48:03.40 & $-03: 31: 44.9$ & $10.15 \pm 4.27$ & $2.5 \pm 1.6$ & $0.70 \pm 0.19$ & $82.1 \pm 30.5$ & $1537 / 2300$ \\
\hline Abell 209 & 0.206 & $01: 31: 52.54$ & $-13: 36: 40.4$ & $19.31 \pm 3.58$ & $3.4 \pm 0.7$ & $0.61 \pm 0.10$ & $-28.6 \pm 9.8$ & $1581 / 2300$ \\
\hline Abell 2261 & 0.224 & $17: 22: 27.18$ & $+32: 07: 57.3$ & $24.80 \pm 3.50$ & $3.7 \pm 0.6$ & $0.78 \pm 0.11$ & $20.9 \pm 14.0$ & $1529 / 2300$ \\
\hline RX J2129.7+0005 & 0.234 & 21:29:39.96 & $+00: 05: 21.2$ & $7.78 \pm 2.43$ & $2.9 \pm 1.2$ & $0.81 \pm 0.14$ & $85.0 \pm 43.8$ & $1477 / 2300$ \\
\hline Abell 611 & 0.288 & 08:00:56.82 & $+36: 03: 23.6$ & $13.44 \pm 3.39$ & $4.2 \pm 1.3$ & $0.56 \pm 0.19$ & $46.8 \pm 9.3$ & $1586 / 1932$ \\
\hline MS2137-2353 & 0.313 & $21: 40: 15.17$ & $-23: 39: 40.2$ & $10.78 \pm 3.17$ & $2.4 \pm 1.0$ & $0.76 \pm 0.17$ & $-53.8 \pm 38.7$ & $1441 / 1760$ \\
\hline RX J2248.7-4431 & 0.348 & $22: 48: 43.96$ & $-44: 31: 51.3$ & $19.81 \pm 5.97$ & $1.6 \pm 0.7$ & $0.51 \pm 0.19$ & $55.5 \pm 12.2$ & $866 / 1440$ \\
\hline MACS J1115.9+0129 & 0.352 & $11: 15: 51.90$ & $+01: 29: 55.1$ & $17.91 \pm 3.81$ & $2.5 \pm 0.7$ & $0.53 \pm 0.14$ & $-32.3 \pm 8.1$ & $1155 / 1440$ \\
\hline MACS J1931.8-2635 & 0.352 & $19: 31: 49.62$ & $-26: 34: 32.9$ & $11.62 \pm 2.84$ & $7.8 \pm 1.7$ & $0.77 \pm 0.18$ & $38.9 \pm 44.9$ & $1818 / 1440$ \\
\hline RX J1532.9+3021 & 0.363 & $15: 32: 53.78$ & $+30: 20: 59.4$ & $7.01 \pm 1.49$ & $7.1 \pm 1.7$ & $0.82 \pm 0.14$ & $52.2 \pm 44.7$ & $1120 / 1440$ \\
\hline MACS J1720.3+ & 0.391 & $17: 20: 16.78$ & $+35: 36: 26.5$ & $11.18 \pm 2.38$ & $5.9 \pm 1.7$ & $0.73 \pm 0.15$ & $24.8 \pm 25.8$ & $1053 / 1292$ \\
\hline MACS J0429.6-0253 & 0.399 & $04: 29: 36.05$ & $-02: 53: 06.1$ & $8.88 \pm 1.70$ & $7.7 \pm 1.6$ & $0.84 \pm 0.12$ & $79.4 \pm 50.4$ & $1081 / 1292$ \\
\hline MACS J1206.2-0847 & 0.440 & $12: 06: 12.15$ & $-08: 48: 03.4$ & $15.05 \pm 3.20$ & $5.8 \pm 1.7$ & $0.78 \pm 0.14$ & $-80.6 \pm 29.9$ & $973 / 1152$ \\
\hline MACS J0329.7-0211 & 0.450 & $03: 29: 41.56$ & $-02: 11: 46.1$ & $12.70 \pm 2.19$ & $5.4 \pm 1.3$ & $0.49 \pm 0.09$ & $-51.7 \pm 8.1$ & $563 / 1020$ \\
\hline RX J1347.5-1145 & 0.451 & $13: 47: 31.05$ & $-11: 45: 12.6$ & $35.40 \pm 5.05$ & $4.5 \pm 0.9$ & $0.58 \pm 0.12$ & $20.9 \pm 7.4$ & $1349 / 1020$ \\
\hline MACS J0744.9+3927 & 0.686 & $07: 44: 52.82$ & $+39: 27: 26.9$ & $17.23 \pm 6.16$ & $2.0 \pm 1.4$ & $0.35 \pm 0.27$ & $-63.6 \pm 6.4$ & $274 / 672$ \\
\hline \multicolumn{9}{|l|}{ High Magnification: } \\
\hline MACS J0416.1-2403 & 0.396 & 04:16:08.38 & $-24: 04: 20.8$ & $11.43 \pm 2.66$ & $2.9 \pm 0.9$ & $0.65 \pm 0.16$ & $45.1 \pm 13.9$ & $867 / 1292$ \\
\hline MACS J1149.5+2223 & 0.544 & $11: 49: 35.69$ & $+22: 23: 54.6$ & $28.86 \pm 5.92$ & $1.5 \pm 0.4$ & $0.37 \pm 0.09$ & $-38.5 \pm 5.8$ & $704 / 896$ \\
\hline MACS J0717.5+3745 & 0.548 & $07: 17: 32.63$ & $+37: 44: 59.7$ & $35.96 \pm 5.43$ & $3.0 \pm 0.6$ & $0.45 \pm 0.09$ & $-56.8 \pm 6.0$ & $729 / 896$ \\
\hline MACS J0647.7+7015 & 0.584 & $06: 47: 50.27$ & $+70: 14: 55.0$ & $11.73 \pm 3.79$ & $2.3 \pm 1.2$ & $0.76 \pm 0.18$ & $77.2 \pm 52.3$ & $574 / 780$ \\
\hline
\end{tabular}

Notes.

${ }^{\text {a }}$ Cluster redshifts were taken from Umetsu et al. (2014).

$\mathrm{b}$ The cluster center represents the location of the brightest cluster galaxy when a single dominant central galaxy is found. Otherwise, for MACS J0717.5+3745 and MACS J0416.1-2403, it is the center of the brightest red-sequence-selected cluster galaxies.

${ }^{\mathrm{c}}$ Projected minor-to-major halo axis ratio $q_{\perp} \leqslant 1$.

${ }^{\mathrm{d}}$ Position angle (PA) of the projected halo major axis measured east of north, defined in the range $\left[-90^{\circ}, 90^{\circ}\right)$.

${ }^{\mathrm{e}}$ Minimum $\chi^{2}$ per degrees of freedom (dof).

subsamples, they found that the X-ray-selected subsample is slightly rounder (typical axis ratio of $\sim 0.9$ versus $\sim 0.8$ measured within an aperture radius of $350 \mathrm{kpc} \mathrm{h}^{-1}$ ), more centrally concentrated, and has smaller centroid shifts than the lensing-selected subsample. In order to understand how typical CLASH clusters are relative to a complete set of simulated clusters of similar mass, Donahue et al. (2016) also compared high-mass halos from nonradiative simulations (Sembolini et al. 2013; Meneghetti et al. 2014) with the CLASH Chandra observations. They found that, overall, both X-ray- and lensing-selected CLASH clusters are rounder than the simulated clusters in terms of the $\mathrm{X}$-ray axis ratio.

Following Umetsu et al. (2014, 2016), we use the location of the brightest cluster galaxy (BCG) as the cluster center (Table 1). On average, the sample exhibits a small positional offset between the BCG and X-ray peak, characterized by an rms offset of $\sigma_{\text {off }} \simeq 30 \mathrm{kpc} h^{-1}$ (Umetsu et al. 2014). For the X-ray-selected subsample, the offset is even smaller, $\sigma_{\text {off }} \simeq 11 \mathrm{kpc}^{-1}$ (Umetsu et al. 2014), because of the CLASH selection function. This level of centering offset is sufficiently small compared to the range of cluster radii of interest (e.g., $r_{2500 c}, r_{500 \mathrm{c}}, r_{200 \mathrm{c}}, r_{200 \mathrm{~m}}$ ), as well as to the effective resolution of our mapmaking (see Section 4.1). Accordingly, smoothing from the miscentering effects (see Johnston et al. 2007 and Umetsu et al. 2011a) is not expected to significantly impact our mass, concentration, and shape measurements for our cluster sample.

Since the clusters in our sample are highly massive $\left(M_{200 c} \simeq 14 \times 10^{14} M_{\odot} h_{70}^{-1}\right.$; Umetsu et al. 2016), they can strongly lens background galaxies into multiple images or giant arcs in their central region. On the basis of deep multiband $H S T$ images, Zitrin et al. (2015) identified many secure sets of multiple-image systems in all CLASH clusters except RX J1532.9+3021 (see Zitrin et al. 2015), our least massive cluster with $M_{200 c} \sim 6 \times 10^{14} M_{\odot} h_{70}^{-1}$ (Tables 2 and 3 of Umetsu et al. 2016). For our sample, we find a median effective Einstein radius ${ }^{30}$ of $\simeq 22^{\prime \prime}$ (Umetsu et al. 2016) for a source redshift of $z=2$. We refer to our companion papers (Chiu et al. 2018; Sereno et al. 2018) for joint analyses of central strong-lensing and 2D weak-lensing constraints.

\subsection{Photometry and Background Selection}

A secure background selection is critical for a cluster weaklensing analysis, so that unlensed foreground and member galaxies do not dilute the lensing signal (Broadhurst et al. 2005b; Medezinski et al. 2010; Okabe et al. 2013; Medezinski et al. 2017). Umetsu et al. (2014) employed the color-color (CC) selection method of Medezinski et al. (2010) to identify background galaxy populations, typically using the Subaru/ Suprime-Cam $B R_{\mathrm{C}} z^{\prime}$ photometry where available (for a summary, see Table 1 of Umetsu et al. 2014), which spans the full optical wavelength range. The photometric zero points were precisely calibrated to an accuracy of $\sim 0.01 \mathrm{mag}$, using the HST photometry of cluster elliptical galaxies and with a set

\footnotetext{
$\overline{30}$ The effective Einstein radius is defined as $\theta_{\text {Ein }}=\sqrt{A_{c} / \pi}$, with $A_{c}$ the area enclosed within the critical curves.
} 
of galaxies having spectroscopic redshifts from the CLASHVLT large spectroscopic program with VIMOS (e.g., Biviano et al. 2013; Annunziatella et al. 2014; Rosati et al. 2014; Grillo et al. 2015; Balestra et al. 2016), which obtained thousands of spectroscopic redshifts for cluster members and intervening galaxies along the line of sight, including lensed background galaxies.

The CC-selection method has been calibrated with evolutionary color tracks of galaxies (Kotulla et al. 2009; Medezinski et al. 2010, 2011; Hanami et al. 2012; Medezinski et al. 2017) and with well-calibrated photometric-redshift (photo- $z$ ) catalogs such as COSMOS (Ilbert et al. 2009; Laigle et al. 2016). For details of our CC selection, we refer the reader to Section 4.4 of Umetsu et al. (2014) and Section 3.2 of Umetsu et al. (2016).

For shear measurements, Umetsu et al. (2014) combined two distinct background populations that encompass the red and blue branches of field galaxies in the $\mathrm{CC}$ plane, having redshift distributions peaked around $z \sim 1$ and $z \sim 2$, respectively (Medezinski et al. 2010, 2011).

Umetsu et al. (2014) used flux-limited samples of red background galaxies at $z \sim 1$ for magnification bias measurements. Faint magnitude cuts were applied in the reddest band to avoid incompleteness near the detection limit. Our CC selection avoids incompleteness at the faint end in the bluer bands (Hildebrandt 2016) because we have correspondingly deeper photometry in the bluer bands. As discussed in Umetsu et al. (2016), this is by design to detect faint red galaxies as proposed by Broadhurst (1995).

The mean lensing depths $\langle\beta\rangle$ and $\left\langle\beta^{2}\right\rangle$ of the respective background samples were estimated using the photo- $z$ 's of individual galaxies determined with the BPZ code (Benítez 2000; Benítez et al. 2004) from our point-spread-function (PSF) corrected photometry typically in five Suprime-Cam bands (see Table 1 of Umetsu et al. 2014). An excellent statistical agreement was obtained between the $\langle\beta\rangle$ estimates from the BPZ measurements in the CLASH fields and those from the COSMOS photo- $z$ catalog, with a median offset of $0.27 \%$ and a field-to-field scatter of $5.0 \%$ (Umetsu et al. 2014).

\subsection{Two-dimensional Weak-lensing Shear Analysis}

\subsubsection{Reduced Shear Field}

We use the 2D reduced shear field averaged on a grid as the primary constraint from our wide-field weak-lensing observations. From shape measurements of background galaxies, the source-averaged reduced shear $g_{n}=g\left(\boldsymbol{\theta}_{n}\right)$ is measured on a regular Cartesian grid of pixels $\left(n=1,2, \ldots, N_{\text {pix }}\right)$ as

$$
g_{n}=\left[\sum_{k} S\left(\boldsymbol{\theta}_{(k)}, \boldsymbol{\theta}_{n}\right) w_{(k)} g_{(k)}\right]\left[\sum_{k} S\left(\boldsymbol{\theta}_{(k)}, \boldsymbol{\theta}_{n}\right) w_{(k)}\right]^{-1}
$$

where $S\left(\boldsymbol{\theta}, \boldsymbol{\theta}^{\prime}\right)$ is a spatial window function, $g_{(k)}$ is an estimate of $g(\boldsymbol{\theta})$ for the $k$ th galaxy at $\boldsymbol{\theta}_{(k)}$, and $w_{(k)}$ is its statistical weight, $w_{(k)}=1 /\left(\sigma_{g(k)}^{2}+\alpha_{g}^{2}\right)$, with $\sigma_{g(k)}^{2}$ the error variance of $g_{(k)}$. Following Umetsu et al. (2014), we choose $\alpha_{g}=0.4$, a typical value of the mean rms $\left(\overline{\sigma_{g}^{2}}\right)^{1 / 2}$ found in Subaru observations (Umetsu et al. 2009; Oguri et al. 2010; Okabe \& Smith 2016).

The theoretical expectation (denoted by a hat symbol) for the estimator (9) is approximated by (Seitz \& Schneider 1997;
Umetsu et al. 2015)

$$
\widehat{g}\left(\boldsymbol{\theta}_{n}\right) \approx \frac{\langle W\rangle_{g} \gamma_{\infty}\left(\boldsymbol{\theta}_{n}\right)}{1-f_{W, g}\langle W\rangle_{g} \kappa_{\infty}\left(\boldsymbol{\theta}_{n}\right)},
$$

where $\langle W\rangle_{g}$ is the source-averaged relative lensing strength (Section 2.2), and $f_{W, g}=\left\langle W^{2}\right\rangle_{g} /\langle W\rangle_{g}^{2}$ is a dimensionless correction factor of order unity. The error variance $\sigma_{g, n}^{2}$ for $g_{n}$ is expressed as

$$
\sigma_{g, n}^{2}=\frac{\sum_{k} S^{2}\left(\boldsymbol{\theta}_{(k)}, \boldsymbol{\theta}_{n}\right) w_{(k)}^{2} \sigma_{g(k)}^{2}}{\left[\sum_{k} S\left(\boldsymbol{\theta}_{(k)}, \boldsymbol{\theta}_{n}\right) w_{(k)}\right]^{2}} .
$$

We adopt the top-hat window of radius $\theta_{\mathrm{f}}$ (Merten et al. 2009; Umetsu et al. 2015), $S\left(\boldsymbol{\theta}, \boldsymbol{\theta}^{\prime}\right)=H\left(\theta_{\mathrm{f}}-\left|\boldsymbol{\theta}-\boldsymbol{\theta}^{\prime}\right|\right)$, with $H(x)$ the Heaviside function defined such that $H(x)=1$ if $x \geqslant 0$ and $H(x)=0$ otherwise. The shape-noise covariance matrix for $g_{\alpha, n}=g_{\alpha}\left(\boldsymbol{\theta}_{n}\right)$ is then given as (Oguri et al. 2010) $)^{31}$

$$
\left(C_{g}^{\text {shape }}\right)_{\alpha \beta, m n}=\frac{1}{2} \delta_{\alpha \beta} \sigma_{g, m} \sigma_{g, n} \xi_{H}\left(\left|\boldsymbol{\theta}_{m}-\boldsymbol{\theta}_{n}\right|\right),
$$

where the indices $\alpha$ and $\beta$ run over the two components of the reduced shear $(\alpha, \beta=1,2), \delta_{\alpha} \beta$ denotes the Kronecker delta, and $\xi_{H}(x)$ is the autocorrelation of a pillbox of radius $\theta_{\mathrm{f}}$ (White et al. 1999; Park et al. 2003; Umetsu et al. 2015),

$$
\xi_{H}(x)=\frac{2}{\pi}\left[\cos ^{-1}\left(\frac{x}{2 \theta_{\mathrm{f}}}\right)-\left(\frac{x}{2 \theta_{\mathrm{f}}}\right) \sqrt{1-\left(\frac{x}{2 \theta_{\mathrm{f}}}\right)^{2}}\right]
$$

for $|x| \leqslant 2 \theta_{\mathrm{f}}$ and $\xi_{H}(x)=0$ for $|x|>2 \theta_{\mathrm{f}}$.

\subsubsection{CLASH Weak-lensing Shear Data}

In this study, we directly measure $2 \mathrm{D}$ reduced shear maps in 20 CLASH cluster fields using the wide-field shear catalogs obtained by the CLASH collaboration (Umetsu et al. 2014). The shear measurement pipeline of Umetsu et al. (2014) is based on the IMCAT package (Kaiser et al. 1995, hereafter KSB) with modifications incorporating the improvements developed by Umetsu et al. (2010).

Briefly summarizing, the key feature in our analysis pipeline is that only those galaxies detected with sufficiently high significance, $\nu_{g}>30$, are used to model the isotropic PSF correction as a function of object size and magnitude. Here, $\nu_{g}$ is the peak detection significance given by the IMCAT peakfinding algorithm HFINDPEAKS. A very similar procedure was employed by the LoCuSS collaboration in their weak-lensing study of 50 clusters based on Subaru/Suprime-Cam data (Okabe \& Smith 2016). Another key feature is that we select those galaxies isolated in projection for the shape measurement, reducing the impact of crowding and blending (for details, see Umetsu et al. 2014). After the close-pair rejection, objects with low detection significance $\nu_{g}<10$ were excluded from our analysis. All galaxies with usable shape measurements are matched with those in our CC-selected samples of background galaxies (Section 3.2), ensuring that each galaxy is detected in both the reddest $\mathrm{CC}$-selection band and the shape measurement band. Applying conservative selection criteria (Section 3.2), Umetsu et al. (2014) find a typical surface number density of

\footnotetext{
31 In Oguri et al. (2010), $\sigma_{g}$ denotes the per-component dispersion due to shape noise.
} 
$n_{\mathrm{g}} \simeq 12$ galaxies $\operatorname{arcmin}^{-2}$ for their weak-lensing-matched background catalogs (Umetsu et al. 2014, their Table 3).

In Appendix A, we show the results of our shape measurement test based on simulated Subaru/Suprime-Cam images provided by M. Oguri. From this test, we find that the reduced shear signal can be recovered up to $\left|g_{\alpha}\right| \simeq 0.3$ with $m_{\alpha} \simeq-0.05$ of the multiplicative calibration bias and $\left|c_{\alpha}\right|<10^{-3}$ of the residual shear offset (for details, see Appendix A), where the observed and true values of the reduced shear are related by (Heymans et al. 2006; Massey et al. 2007)

$$
g_{\alpha}^{\text {obs }}=\left(1+m_{\alpha}\right) g_{\alpha}^{\text {true }}+c_{\alpha} .
$$

Umetsu et al. (2014) included for each galaxy a shear calibration factor with $m_{1}=m_{2}=-0.05$ to account for residual calibration. The degree of multiplicative bias $m_{\alpha}$ depends on the seeing conditions and the PSF properties (Figure 10), so that the variation with the PSF properties limits the shear calibration accuracy to $\delta m_{\alpha} \sim 0.05$ (Umetsu et al. 2012, Section 3.3). We note that the same simulation data set was used by the LoCuSS collaboration (Okabe \& Smith 2016) to test their shape measurement pipeline, and Okabe \& Smith (2016) found a similar level of shear calibration bias ( $m_{\alpha} \sim-0.03$ ) from their mock observations.

For all cluster fields in our sample, the estimated values for $\langle\beta\rangle_{g}$ and $f_{W, g}$ are summarized in Table 3 of Umetsu et al. (2014). The calibration uncertainty in $\langle\beta\rangle_{g}$ is marginalized over in our joint analysis of shear and magnification data (Section 3.5).

\subsection{Weak-lensing Magnification Analysis}

\subsubsection{Flux Magnification Bias}

Lensing magnification can influence the observed surface number density of background sources, amplifying their apparent fluxes and expanding the area of sky (Hildebrandt et al. 2011; Umetsu et al. 2011b; Morrison et al. 2012; GarciaFernandez et al. 2016; Tudorica et al. 2017). The former effect increases the number of sources detectable above the limiting flux, whereas the latter reduces the effective observing area in the source plane, reducing the number of sources per solid angle. The net effect is known as magnification bias (Broadhurst et al. 1995) and depends on the slope of the intrinsic source luminosity function. Since a given flux limit corresponds to different luminosities at different source redshifts, number counts of distinctly different source populations probe different regimes of magnification bias (Umetsu 2013).

Deep multiband photometry can be used to sample the faint end of the luminosity function of red quiescent galaxies lying at $z \sim 1$ (e.g., Ilbert et al. 2010). For such a source population, the effect of magnification bias is dominated by the geometric area distortion, because there are relatively few fainter galaxies that can be magnified into the flux-limited sample. This effect results in a net depletion of source counts, a phenomenon known as negative magnification bias or weak-lensing depletion (e.g., Broadhurst 1995; Taylor et al. 1998; Broadhurst et al. 2005b; Umetsu \& Broadhurst 2008; Umetsu et al. 2011b, 2012, 2014, 2015; Coe et al. 2012; Ford et al. 2012; Medezinski et al. 2013; Radovich et al. 2015; Ziparo et al. 2016; Wong et al. 2017). A practical advantage of this technique, at the expense of very deep multicolor imaging, is that the effect is not sensitive to the exact form of the source luminosity function (Umetsu et al. 2014).

In the weak-lensing regime, the shift in magnitude $\delta m=2.5 \log _{10} \mu$ due to magnification is small compared to the range in which the slope of the luminosity function varies. The number counts can then be approximated by a power law at the limiting magnitude $m_{\mathrm{lim}}$. The expectation value (denoted by a hat symbol) for the lensed counts at source redshift $z$ is then expressed as (Broadhurst et al. 1995)

$$
\widehat{N_{\mu}}\left(\boldsymbol{\theta}, z \mid<m_{\lim }\right)=\overline{N_{\mu}}\left(z \mid<m_{\lim }\right) \Delta_{\mu}(\boldsymbol{\theta}, z)^{1-2.5 s},
$$

with $\bar{N}_{\mu}\left(z \mid<m_{\text {lim }}\right)$ the unlensed mean counts per cell and $s$ the logarithmic count slope evaluated at $m=m_{\text {lim }}{ }^{32}$

$$
s=\left.\frac{d \log _{10} \bar{N}(z \mid<m)}{d m}\right|_{m_{\mathrm{lim}}} .
$$

A count depletion (enhancement) results when $s<0.4(>0.4)$.

Following Umetsu et al. (2014), we interpret the observed source-averaged magnification bias as

$$
\begin{aligned}
\widehat{b}_{\mu}(\boldsymbol{\theta}) & \equiv \frac{\widehat{N}_{\mu}\left(\boldsymbol{\theta} \mid<m_{\mathrm{lim}}\right)}{\bar{N}_{\mu}\left(<m_{\mathrm{lim}}\right)} \approx \Delta_{\mu}(\boldsymbol{\theta})^{1-2.5 s_{\mathrm{eff}}}, \\
\Delta_{\mu}(\boldsymbol{\theta}) & =\frac{\int_{0}^{\infty} d z \bar{N}_{\mu}\left(z \mid<m_{\mathrm{lim}}\right) \Delta_{\mu}(\boldsymbol{\theta}, z)}{\int_{0}^{\infty} d z \bar{N}_{\mu}\left(z \mid<m_{\mathrm{lim}}\right)} \\
& \approx\left[1-\langle W\rangle_{\mu} \kappa_{\infty}(\boldsymbol{\theta})\right]^{2}-\langle W\rangle_{\mu}^{2}\left|\gamma_{\infty}(\boldsymbol{\theta})\right|^{2},
\end{aligned}
$$

where $\quad \bar{N}_{\mu}\left(<m_{\text {lim }}\right)=\int_{0}^{\infty} d z \bar{N}_{\mu}\left(z \mid<m_{\text {lim }}\right), \quad s_{\text {eff }}=d \log _{10} \bar{N}_{\mu}(<$ $m) /\left.d m\right|_{m_{\mathrm{lim}}}$, and $\langle W\rangle_{\mu}$ is the source-averaged relative lensing strength (Section 2.2). Equation (17) gives a good approximation for depleted populations with $s_{\text {eff }} \ll 0.4$ (for details, see Appendix A.2 of Umetsu 2013). For simplicity, we write $N_{\mu}(\boldsymbol{\theta})=$ $N_{\mu}\left(\boldsymbol{\theta} \mid<m_{\mathrm{lim}}\right)$ and $\bar{N}_{\mu}=\bar{N}_{\mu}\left(<m_{\mathrm{lim}}\right)$. In the weak-lensing limit, Equation (17) reads $\widehat{b}_{\mu}-1 \approx\left(5 s_{\text {eff }}-2\right)\langle W\rangle_{\mu} \kappa_{\infty}$.

We azimuthally average the observed counts $N_{\mu}(\boldsymbol{\theta})$ in clustercentric, circular annuli and calculate the surface number density $\left\{n_{\mu, i}\right\}_{i=1}^{N_{\text {bin }}}$ of background galaxies as (Umetsu et al. 2015, 2016)

$$
n_{\mu, i}=\frac{1}{\left(1-f_{\text {mask }, i}\right) \Omega_{\text {cell }}} \sum_{m} \mathcal{P}_{i m} N_{\mu}\left(\boldsymbol{\theta}_{m}\right),
$$

with $\Omega_{\text {cell }}$ the solid angle per cell and $\mathcal{P}_{i m}=\left(\sum_{m} A_{m i}\right)^{-1} A_{m i}$ the projection matrix normalized in each annulus by $\sum_{m} \mathcal{P}_{i m}=1$. Here, $A_{m i}$ is the area fraction of the $m$ th cell lying within the $i$ th radial bin $\left(0 \leqslant A_{m i} \leqslant 1\right)$, and $f_{\text {mask }, i}$ is the mask correction factor for the $i$ th radial bin due to saturated objects, foreground and cluster galaxies, and bad pixels (for details, see Section 3.4 of Umetsu et al. 2016).

The theoretical expectation for the estimator (18) is

$$
\widehat{n}_{\mu, i}=\bar{n}_{\mu} \sum_{m} \mathcal{P}_{i m} \Delta_{\mu}\left(\boldsymbol{\theta}_{m}\right)^{1-2.5 s_{\mathrm{eff}}}
$$

with $\bar{n}_{\mu}=\bar{N}_{\mu} / \Omega_{\text {cell }}$.

The choice of annular constraints rather than pixelated ones is mainly because magnification constraints are by far noisier

\footnotetext{
$\overline{32}$ In the literature, $\alpha \equiv-d \log \bar{N}(>F) / d \log F=2.5 s$ in terms of the limiting flux $F$ is often used instead of $s$ (e.g., Umetsu et al. 2015).
} 
than shear measurements, especially due to the local clustering noise (see Umetsu \& Broadhurst 2008). An optimal choice of the resolution is to have per-element signal-to-noise ratio of order unity or above. This is satisfied by azimuthally averaging noisy count measurements, while it allows us to estimate the variance due to angular clustering at large clustercentric distances.

\subsubsection{CLASH Weak-lensing Magnification Data}

We use the CLASH weak-lensing magnification measurements obtained using flux-limited samples of red background galaxies as published in Umetsu et al. (2014). They measured the magnification effects in $N_{\text {bin }}=10$ log-spaced circular annuli centered on the cluster. The radial bins range from $0{ }^{\prime} \cdot 9$ to $16^{\prime}$ for all clusters, except 0 '9 to $14^{\prime}$ for RX J2248.7-4431 observed with ESO/WFI. Our magnification analysis begins at $\theta_{\min }=0.9$, which is sufficiently large compared to the range of effective Einstein radii for our sample (Zitrin et al. 2015; Umetsu et al. 2016). The magnification profiles $\left\{n_{\mu, i}\right\}_{i=1}^{N_{\text {bin }}}$ used in the present work are presented in Figure 2 of Umetsu et al. (2014).

Here we briefly describe the magnification analysis performed in Umetsu et al. (2014). Their analysis was limited to the $24^{\prime} \times 24^{\prime}$ region centered on the cluster. They accounted for the Poisson, intrinsic clustering, and additional systematic contributions to the total uncertainty $\sigma_{\mu}$. The clustering noise term $\sigma_{\mu, i}^{\text {int }}$ was estimated in each circular annulus from the variance due to variations of the counts along the azimuthal direction. Besides, a positive tail of $>\nu \sigma$ cells with $\nu=2.5$ was removed in each circular annulus by iterative $\sigma$ clipping to reduce the bias due to intrinsic angular clustering of red galaxies. We checked that the clipping threshold chosen is sufficiently high compared to the maximum variations of the magnification signal due to halo ellipticity (typically, $|\delta \kappa(\theta)| /\langle\kappa(\theta)\rangle \lesssim 0.5$ for a projected halo axis ratio of $\geqslant 0.6)$. The Poisson noise term $\sigma_{\mu, i}^{\text {stat }}$ was estimated from the clipped mean counts in each annulus. The difference between the mean counts estimated with and without $\sigma$ clipping was taken as a systematic error, $\sigma_{\mu, i}^{\text {sys }}=\left|n_{\mu, i}^{(\nu)}-n_{\mu, i}^{(\infty)}\right| / \nu$, where $n_{\nu, i}^{(\nu)}$ and $n_{\mu, i}^{(\infty)}$ denote the clipped and unclipped mean counts in the $i$ th annulus, respectively. Finally, these errors were combined in quadrature as

$$
\sigma_{\mu, i}^{2}=\left(\sigma_{\mu, i}^{\mathrm{int}}\right)^{2}+\left(\sigma_{\mu, i}^{\mathrm{stat}}\right)^{2}+\left(\sigma_{\mu, i}^{\mathrm{sys}}\right)^{2} .
$$

Our magnification bias measurements are stable and insensitive to the particular choice of $\nu$ because of the inclusion of the $\sigma_{\mu}^{\text {sys }}$ term in the error analysis.

Masking of observed sky was accounted and corrected for using the method of (Umetsu et al. 2011b, Method B of Appendix A), which can be fully automated once the configuration parameters of SExtractor (Bertin \& Arnouts 1996) are optimally tuned (Umetsu et al. 2011b, 2014). Chiu et al. (2016) adopted this method to estimate the masked area fraction in their magnification analysis and found that the SExtractor configuration of Umetsu et al. (2011b) is optimal for their data taken with Megacam on the Magellan Clay telescope.

The count normalization and slope parameters $\left(\bar{n}_{\mu}, s_{\mathrm{eff}}\right)$ were estimated in the cluster outskirts (Umetsu et al. 2014). ${ }^{33}$ The

\footnotetext{
33 As discussed in Umetsu et al. (2014, their Section 7.4.2), the 2-halo term does not cause bias in the reconstruction, because the range of the uniform prior on $\bar{n}_{\mu}$ is sufficiently wide.
}

mask-corrected magnification bias profile $b_{\mu, i}=n_{\mu, i} / \bar{n}_{\mu}$ is proportional to $\left(1-f_{\text {mask,back }}\right) /\left(1-f_{\text {mask }, i}\right) \equiv 1+\Delta f_{\text {mask }, i}$, with $f_{\text {mask,back }}$ estimated in the background region (see Umetsu et al. 2014, 2016). The effect of the mask correction is thus sensitive to the difference of the $f_{\text {mask }}$ values, which is insensitive to the particular choice of the SExtractor configuration parameters. The typical variation of $\Delta f_{\text {mask }, i}$ across the full radial range is $\sim 5 \%$ (see also Chiu et al. 2016), much smaller than the typical magnification signal $\delta n_{\mu} / \bar{n}_{\mu} \sim-0.3$ in the innermost bin. Accordingly, the systematic uncertainty on the mask correction is likely negligible.

The estimated values and errors for $\langle\beta\rangle_{\mu}, \bar{n}_{\mu}$, and $s_{\text {eff }}$ are summarized in Table 4 of Umetsu et al. (2014). The values of $s_{\text {eff }}$ span the range $[0.11,0.20]$ with a mean of $\left\langle s_{\text {eff }}\right\rangle=0.153$ and a typical fractional uncertainty of $33 \%$ per cluster field. We marginalize over the calibration parameters $\left(\langle\beta\rangle_{\mu}, \bar{n}_{\mu}, s_{\text {eff }}\right)$ for each cluster in our joint likelihood analysis of shear and magnification (Section 3.5).

\subsection{Mass Reconstruction Algorithm}

To perform a mass reconstruction, we use the inversion algorithm developed by Umetsu et al. (2015), who generalized the cluster lensing mass inversion (CLUMI) code of Umetsu (2013; see also Umetsu et al. 2011b) into a 2D description of the pixelized mass distribution. This free-form method combines a spatial shear pattern $\left(g_{1}(\boldsymbol{\theta}), g_{2}(\boldsymbol{\theta})\right)$ with azimuthally averaged magnification measurements $\left\{n_{\mu, i}\right\}_{i=1}^{N_{\text {bin }}}$, which impose a set of azimuthally integrated constraints on the $\Sigma$ field, thus effectively breaking the mass-sheet degeneracy. The CLUMI-2D algorithm takes full account of the nonlinear subcritical regime of gravitational lensing properties.

According to Bayes' theorem, given a model $\boldsymbol{\lambda}$ and observed data $\boldsymbol{d}$, the joint posterior probability $P(\boldsymbol{\lambda} \mid \boldsymbol{d})$ is proportional to the product of the likelihood $\mathcal{L}(\lambda) \equiv P(\boldsymbol{d} \mid \boldsymbol{\lambda})$ and the prior probability $P(\lambda)$. In our inversion problem, $\lambda$ represents a signal vector containing the pixelized mass coefficients $s=\left\{\Sigma_{n}\right\}_{n=1}^{N_{\text {pix }}}$ (Section 2.3) and calibration nuisance parameters $\boldsymbol{c}$ (Section 3.5.3), so that $\boldsymbol{\lambda} \equiv(\boldsymbol{s}, \boldsymbol{c})$.

We write the likelihood function $\mathcal{L}$ for combined weaklensing data $\boldsymbol{d}$ as a product of two separate likelihoods, $\mathcal{L}=\mathcal{L}_{g} \mathcal{L}_{\mu}$, with $\mathcal{L}_{g}$ and $\mathcal{L}_{\mu}$ the likelihood functions for shear and magnification, respectively. This implicitly assumes that the cross-covariance between shear and magnification due to projected uncorrelated large-scale structure (LSS) is ignored (see Sections 3.5.1 and 3.5.2). In our measurements, the uncertainty is dominated by observational measurement errors at all scales, as shown in Figure 1 of Umetsu et al. (2016), so that the contribution from this cosmic cross-term is not expected to significantly impact our results. We assume that the observational errors follow a Gaussian distribution, so that $\mathcal{L} \propto \exp \left(-\chi^{2} / 2\right)$, with $\chi^{2}$ the standard misfit statistic.

\subsubsection{Shear Log-likelihood Function}

The $\log$-likelihood function $l_{g} \equiv-\ln \mathcal{L}_{g}$ for 2D shear data can be written (ignoring constant terms) as (Oguri et al. 2010)

$$
\begin{aligned}
l_{g}(\boldsymbol{\lambda})= & \frac{1}{2} \sum_{m, n=1}^{N_{\text {pix }}} \sum_{\alpha, \beta=1}^{2}\left[g_{\alpha, m}-\widehat{g}_{\alpha, m}(\boldsymbol{\lambda})\right]\left(\mathcal{W}_{g}\right)_{\alpha \beta, m n} \\
& \times\left[g_{\beta, n}-\widehat{g}_{\beta, n}(\boldsymbol{\lambda})\right],
\end{aligned}
$$


where $\widehat{g}_{\alpha, m}(\boldsymbol{\lambda})$ is the theoretical expectation for $g_{\alpha, m}=g_{\alpha}\left(\boldsymbol{\theta}_{m}\right)$, and $\left(\mathcal{W}_{g}\right)_{\alpha \beta, m n}$ is the shear weight matrix,

$$
\left(\mathcal{W}_{g}\right)_{\alpha \beta, m n}=M_{m} M_{n}\left(C_{g}^{-1}\right)_{\alpha \beta, m n} .
$$

Here, $M_{m}$ is a mask weight, defined such that $M_{m}=0$ if the $m$ th cell is masked out and $M_{m}=1$ otherwise, and $C_{g}$ is the shear covariance matrix. We account for contributions from the shape covariance $C_{g}^{\text {shape }}$ and the cosmic covariance $C_{g}^{\text {lss }}$ due to uncorrelated LSS projected along the line of sight as

$$
\left(C_{g}\right)_{\alpha \beta, m n}=\left(C_{g}^{\text {shape }}\right)_{\alpha \beta, m n}+\left(C_{g}^{\mathrm{lss}}\right)_{\alpha \beta, m n},
$$

where $\left(C_{g}^{\mathrm{lss}}\right)_{\alpha \beta, m n}=\xi_{\alpha \beta}^{\mathrm{lss}}\left(\left|\boldsymbol{\theta}_{m}-\boldsymbol{\theta}_{n}\right|\right)$ with $\xi_{\alpha \beta}^{\mathrm{lss}}=\xi_{\beta \alpha}^{\mathrm{lss}}(\alpha, \beta=1$, 2) the cosmic shear correlation function ( $\mathrm{Hu} \&$ White 2001 ; Oguri et al. 2010). We compute the elements of the $C_{g}^{\mathrm{lss}}$ matrix for a given source population, using the nonlinear matter power spectrum of Smith et al. (2003) for the Wilkinson Microwave Anisotropy Probe (WMAP) seven-year cosmology (Komatsu et al. 2011). For each cluster, we use the effective mean source redshift ( $\bar{z}_{\text {eff }}$; Table 3 of Umetsu et al. 2014) estimated with our multiband photometric redshifts.

\subsubsection{Magnification Log-likelihood Function}

Similarly, the log-likelihood function for magnification bias data $l_{\mu} \equiv-\ln \mathcal{L}_{\mu}$ is written as (Umetsu et al. 2015)

$$
l_{\mu}(\boldsymbol{\lambda})=\frac{1}{2} \sum_{i=1}^{N_{\text {bin }}}\left[n_{\mu, i}-\widehat{n}_{\mu, i}(\boldsymbol{\lambda})\right]\left(\mathcal{W}_{\mu}\right)_{i j}\left[n_{\mu, j}-\widehat{n}_{\mu, j}(\boldsymbol{\lambda})\right],
$$

where $\widehat{n}_{\mu, i}(\boldsymbol{\lambda})$ is the theoretical expectation for the observed counts $n_{\mu, i}$, and $\left(\mathcal{W}_{\mu}\right)_{i j}$ is the magnification weight matrix, $\mathcal{W}_{\mu}=C_{\mu}^{-1}$, with $C_{\mu}$ the corresponding covariance matrix,

$$
\left(C_{\mu}\right)_{i j}=\sigma_{\mu}^{2} \delta_{i j}+\left(C_{\mu}^{\mathrm{lss}}\right)_{i j}
$$

The diagonal term in Equation (25) is responsible for the observational errors, and the bin-to-bin covariance matrix $C_{\mu}^{\mathrm{lss}}$ accounts for the cosmic noise contribution due to projected uncorrelated LSS, $\left(C_{\mu}^{\mathrm{lss}}\right)_{i j}=\left[\left(5 s_{\mathrm{eff}}-2\right) \bar{n}_{\mu}\right]^{2}\left(C_{\kappa}^{\mathrm{lss}}\right)_{i j}$, where $C_{\kappa}^{\mathrm{lss}}$ is the cosmic convergence matrix (Umetsu et al. 2011a). We compute the elements of the $C_{\kappa}^{\mathrm{lss}}$ matrix for a given source redshift $\left(\bar{z}_{\mathrm{eff}}\right.$; Table 4 of Umetsu et al. 2014) in a similar manner to those of the $C_{g}^{\mathrm{lss}}$ matrix (Section 3.5.1). We evaluate the $C_{\mu}^{\text {lss }}$ matrix by fixing the values of $\bar{n}_{\mu}$ and $s_{\text {eff }}$ to the observed ones (Section 3.4.2).

The $l_{\mu}$ function sets azimuthally integrated constraints on the projected mass distribution and provides the otherwise unconstrained normalization of $\Sigma(\boldsymbol{R})$ over a set of concentric annuli where magnification measurements are available. In this algorithm, no assumption is made about the azimuthal symmetry or isotropy of $\Sigma(\boldsymbol{R})$. We use Monte Carlo integration to compute the projection matrix $\mathcal{P}_{\text {im }}$ (Equation (18)) of size $N_{\text {bin }} \times N_{\text {pix }}$, which is needed to predict $\left\{\widehat{n}_{\mu, i}(\boldsymbol{\lambda})\right\}_{i}^{N_{\text {bin }}}{ }_{1}$ for a given model $\boldsymbol{\lambda}=(\boldsymbol{s}, \boldsymbol{c})$.

\subsubsection{Calibration Parameters}

We account for the calibration uncertainty in the observational nuisance parameters,

$$
\boldsymbol{c}=\left(\langle W\rangle_{g}, f_{W, g},\langle W\rangle_{\mu}, \bar{n}_{\mu}, s_{\mathrm{eff}}\right) .
$$

To this end, we include in our joint-likelihood analysis Gaussian priors on $c$ with mean values and errors estimated from data.

\subsubsection{Best-fit Solution and Covariance Matrix}

The $\log$-posterior function $F(\boldsymbol{\lambda})=-\ln P(\boldsymbol{\lambda} \mid \boldsymbol{d})$ is expressed as a linear sum of the log-likelihood and prior terms. For each cluster, we find the global maximum of the joint posterior probability distribution over $\lambda$ by minimizing $F(\boldsymbol{\lambda})$ with respect to $\boldsymbol{\lambda}$. In the CLUMI-2D implementation of Umetsu et al. (2015), we use the conjugate-gradient method (Press et al. 1992) to find the global solution $\widehat{\lambda}$. We employ an analytic expression for the gradient function $\nabla F(\boldsymbol{\lambda})$ obtained in the nonlinear, subcritical regime (Appendix B).

To quantify the reconstruction errors, we evaluate the Fisher matrix at $\boldsymbol{\lambda}=\widehat{\boldsymbol{\lambda}}$ as

$$
\mathcal{F}_{p p^{\prime}}=\left.\left\langle\frac{\partial^{2} F(\boldsymbol{\lambda})}{\partial \lambda_{p} \partial \lambda_{p^{\prime}}}\right\rangle\right|_{\widehat{\lambda}},
$$

where the angular brackets denote an ensemble average, and the indices $\left(p, p^{\prime}\right)$ run over all model parameters $\boldsymbol{\lambda}=(\boldsymbol{s}, \boldsymbol{c})$. The error covariance matrix of the reconstructed parameters is obtained by $C=\mathcal{F}^{-1}$. We note that the reconstructed mass pixels are correlated primarily because the relation between the shear and convergence is nonlocal (Equation (2)). Additionally, the effects of spatial averaging (Equation (13)) and cosmic noise (Sections 3.5.1 and 3.5.2) produce a covariance between different pixels. In our analysis, the effects of correlated errors are modeled analytically (i.e., $\xi_{H}, \xi^{\mathrm{lss}}, C_{\kappa}^{\mathrm{lss}}$ ).

\subsection{Major Differences from Previous Work}

The present sample of 20 CLASH clusters has been analyzed by Umetsu et al. (2014, 2016) using high-quality CLASH lensing data sets. In what follows, we summarize the major differences of our analysis from these previous studies, which focused on reconstructing $\Sigma(R)$ profiles from azimuthally averaged lensing measurements.

First of all, this work represents a $2 \mathrm{D}$ generalization of the Umetsu et al. (2014) weak-lensing analysis based on their background-selected shear catalogs. Both studies use identical sets of azimuthally averaged magnification constraints (Section 3.4) as input for respective mass reconstructions. In addition to the measurement error and cosmic noise contributions, Umetsu et al. (2014) accounted for systematic uncertainties $C^{\text {sys }}$ due to the residual mass-sheet degeneracy. This uncertainty was estimated in each $\Sigma$ bin as a difference between the global (joint) and local (marginal) posterior solutions. On the other hand, owing to the large number of parameters involved $\left(48^{2}+5=2309\right)$, we do not directly sample posterior probability distributions (Section 3.5.4), and thus we are not able to include the $C^{\text {sys }}$ term in the present analysis. However, as we will see in Section 5.1, our cluster mass measurements are highly consistent with those of Umetsu et al. (2014), with no evidence for systematic offsets in the mass determinations.

Umetsu et al. (2016) combined the wide-field shear and magnification constraints of Umetsu et al. (2014) with central $H S T$ constraints in the form of the enclosed projected mass $M_{2 \mathrm{D}}(<\theta)$, which was derived from detailed mass models of Zitrin et al. (2015) based on their joint analysis of HST 
strong- and weak-lensing data sets. The strong-lensing, weaklensing shear and magnification constraints were combined a posteriori to reconstruct azimuthally averaged $\Sigma(R)$ profiles for the 20 individual clusters. In addition to the inclusion of the HST data, an important difference between the two studies is that Umetsu et al. (2016) included the intrinsic signal covariance matrix $C^{\text {int }}$ (Gruen et al. 2015) in their error analysis, as well as the $C^{\text {sys }}$ term. Here, the $C^{\text {int }}$ matrix accounts for the variations of the projected cluster lensing signal due to the intrinsic scatter in the $c-M$ relation, ${ }^{34}$ the halo asphericity, and the presence of correlated halos (Gruen et al. 2015). This contribution is particularly important at small cluster radii, and hence in the inner HST region (see Figure 1 of Umetsu et al. 2016). In the CLUMP-3D program, we explicitly account for the effects of triaxiality (in particular, halo elongation along the line of sight) in the mass modeling by simultaneously constraining the cluster mass, concentration, triaxial shape, and orientation from Bayesian inference (Sereno et al. 2017b). We defer such full triaxial analyses to our companion papers (Chiu et al. 2018; Sereno et al. 2018).

\section{Results}

\subsection{Weak-lensing Mapmaking}

Following the methodology outlined in Section 3, we analyze our weak-lensing shear and magnification data sets and perform reconstructions of the 2D lensing fields for our sample of 20 CLASH clusters. For magnification measurements, we have 10 azimuthally averaged constraints $\left\{n_{\mu, i}\right\}_{i=1}^{N_{\text {bin }}}$ in log-spaced clustercentric annuli (Section 3.4), as obtained by Umetsu et al. (2014). To derive reduced shear maps $\left(g_{1}(\boldsymbol{\theta})\right.$, $g_{2}(\boldsymbol{\theta})$ ), we use a top-hat window of $\theta_{\mathrm{f}}=0$.'4 (Section 3.3) to average galaxy ellipticities into a regular grid of $N_{\text {pix }}=48 \times 48$ pixels, each with $\Delta \theta=0.5$ spacing. The shear grid covers a $24^{\prime} \times 24^{\prime}$ region centered on the cluster (Table 1), where Umetsu et al. (2014) obtained the magnification measurements. The filter size corresponds to an effective resolution of $2 \mathrm{D}_{1} \theta_{\mathrm{f}} \simeq 180 \mathrm{kpc} h^{-1}$ at the median redshift of the sample, $z=0.377$. To avoid potential systematic errors, we exclude from our analysis those pixels having no usable background galaxies and the innermost central pixels where $\Sigma$ can be greater than or close to the critical value $\Sigma_{\mathrm{c}}$, ensuring that all of the measurements are in the subcritical regime.

For each cluster, we pixelize the $\kappa_{\infty}$ and $\gamma_{\infty}$ fields on a $N_{\text {pix }}=48 \times 48$ grid covering the central $24^{\prime} \times 24^{\prime}$ region. The model $\boldsymbol{\lambda}=(\boldsymbol{s}, \boldsymbol{c})$ is specified by $N_{\text {pix }}=48^{2}$ mass coefficients, $\boldsymbol{s}=\left\{\Sigma_{n}\right\}_{n=1}^{N_{\text {pix }}}$, and a set of five calibration parameters $\boldsymbol{c}$ (Equation (26)) to marginalize over. We utilize the FFTW implementation of fast Fourier transforms (FFTs) to compute $\gamma_{\infty}(\boldsymbol{\theta})$ from $\kappa_{\infty}(\boldsymbol{\theta})$ using Equation (8). To minimize spurious aliasing effects from the periodic boundary condition, the maps are zero-padded to twice the original length in each spatial dimension (e.g., Seljak 1998; Umetsu \& Broadhurst 2008; Umetsu et al. 2015). In Figure 1, we show the reconstructed $\Sigma$ field centered on MACS J0329.7-0211 as an example of our weak-lensing mass reconstruction.

\footnotetext{
34 As noted by Umetsu et al. (2016), when simultaneously determining the mass and concentration for an individual cluster, the contribution from the intrinsic $c-M$ scatter should be excluded from $C^{\text {int }}$. We note that the effect of the $c-M$ scatter becomes important only at $\theta \lesssim 2^{\prime}$ (Gruen et al. 2015).
}

\subsection{Characterizing the Cluster Mass Distribution}

\subsubsection{Spherical and Elliptical Mass Models}

We model the radial mass distribution in galaxy clusters with a Navarro-Frenk-White (NFW) density profile, motivated by cosmological $N$-body simulations (Navarro et al. 1996, 1997) as well as by direct lensing observations (e.g., Newman et al. 2013; Okabe et al. 2013; Umetsu et al. 2014, 2016; Niikura et al. 2015; Okabe \& Smith 2016; Umetsu \& Diemer 2017). The radial dependence of the spherical NFW density profile is given by (Navarro et al. 1996)

$$
\rho(r)=\frac{\rho_{\mathrm{s}}}{\left(r / r_{\mathrm{s}}\right)\left(1+r / r_{\mathrm{s}}\right)^{2}},
$$

with $\rho_{\mathrm{s}}$ the characteristic density parameter and $r_{s}$ the characteristic scale radius at which the logarithmic slope of the density profile equals -2 . We specify the spherical NFW model with the halo mass, $M_{200 c}$, and the concentration parameter, $c_{200 \mathrm{c}} \equiv r_{200 \mathrm{c}} / r_{\mathrm{s}}$.

The surface mass density $\Sigma(R)$ as a function of projected clustercentric radius $R$ is given by projecting $\rho(r)$ along the line of sight. We employ an analytic expression given by Wright $\&$ Brainerd (2000) for the radial dependence of the projected NFW profile, $\Sigma\left(R \mid M_{200 \mathrm{c}}, c_{200 \mathrm{c}}\right)$, which provides a good approximation for the projected halo model within a couple of virial radii (Oguri \& Hamana 2011) and an excellent description of the projected mass distribution in clusters at $R \lesssim r_{200 m}$ (Umetsu et al. 2016; Umetsu \& Diemer 2017).

We follow the prescription given by Oguri et al. $(2010,2012)$ to construct an elliptical NFW (eNFW hereafter) model, which can be used to characterize the morphology of projected triaxial ellipsoids. To this end, we introduce the mass ellipticity, $\epsilon$, in isodensity contours of the projected NFW profile $\Sigma\left(R \mid M_{200 c}, c_{200 c}\right)$ as (Evans \& Bridle 2009; Oguri et al. 2010; Umetsu et al. 2012; Medezinski et al. 2016)

$$
R^{2}=X^{\prime 2}(1-\epsilon)+Y^{\prime 2} /(1-\epsilon),
$$

where our definition of the ellipticity is $\epsilon=1-q_{\perp}$, with $q_{\perp} \leqslant 1$ the projected minor-to-major axis ratio of isodensity contours, and we have chosen the coordinate system $\left(X^{\prime}, Y^{\prime}\right)$ centered on the cluster halo, such that the $X^{\prime}$ axis is aligned with the major axis of the projected ellipse. Note that the isodensity area is $\pi R^{2}$, so that $R$ represents the geometric mean radius of the isodensity ellipse. Accordingly, the $M_{200 c}$ and $c_{200 \mathrm{c}}$ parameters in the eNFW model can be interpreted as respective spherical equivalent quantities. ${ }^{35}$ In this work, we adopt the observer's coordinate system in which the $X$ - and $Y$ axes are aligned with the west and north, respectively. With this coordinate system, the position angle (PA) of the projected major axis is measured east of north. An alternative definition for the projected ellipticity is $e=\left(1-q_{\perp}^{2}\right) /\left(1+q_{\perp}^{2}\right)$ (e.g., Evans \& Bridle 2009).

\subsubsection{Bayesian Inference}

We use a Bayesian Markov Chain Monte Carlo (MCMC) method to obtain an accurate inference of the eNFW parameters from our 2D weak-lensing data (Section 4.1). In

\footnotetext{
35 This corresponds to a triaxial model with a special geometric configuration, where $f_{\text {geo }} \equiv e_{\|} / \sqrt{q_{\perp}}=1$, with $e_{\|}$the 3D halo elongation parameter of Umetsu et al. (2015).
} 
this study and subsequent companion papers (Chiu et al. 2018; Sereno et al. 2018), we perform model fitting to the 2D surface mass density data, rather than fitting directly to the combined shear and magnification data sets. ${ }^{36}$ This allows consistency checks with existing codes used in previous work (e.g., Oguri et al. 2005; Umetsu \& Broadhurst 2008; Morandi et al. 2011; Sereno \& Umetsu 2011; Sereno et al. 2013; Umetsu et al. 2015; Sereno et al. 2017b) that also used weak-lensing $\Sigma$ map data for 2D and 3D mass modeling. The mass maps obtained in this work are also useful for further studies of substructures in the context of the multiwavelength CLUMP-3D program.

The projected eNFW model is specified by four parameters, $\boldsymbol{p}$ $=\left(M_{200 \mathrm{c}}, c_{200 \mathrm{c}}, q_{\perp}, \mathrm{PA}\right)$. We use uniform prior distributions for the projected axis ratio and position angle in the range $0.1 \leqslant$ $q_{\perp} \leqslant 1$ and $-90^{\circ} \leqslant \mathrm{PA}<90^{\circ}$ (Oguri et al. 2010). Following Umetsu et al. (2014, 2016), we assume log-uniform priors for $M_{200 \mathrm{c}}$ and $c_{200 \mathrm{c}}$ in the range $0.1 \leqslant M_{200 \mathrm{c}} /\left(10^{15} M_{\odot} h^{-1}\right) \leqslant 10$ and $0.1 \leqslant c_{200 c} \leqslant 10$. As found by Sereno et al. (2015) and Umetsu et al. (2016), the mass and concentration estimates for the CLASH sample are not sensitive to the choice of the priors, thanks to the deep high-quality weak-lensing observations. The $\chi^{2}$ function for our observations is

$$
\chi^{2}(\boldsymbol{p})=\sum_{m, n=1}^{N_{\mathrm{pix}}}\left[\Sigma_{m}-\widehat{\Sigma}_{m}(\boldsymbol{p})\right]\left(C^{-1}\right)_{m n}\left[\Sigma_{n}-\widehat{\Sigma}_{n}(\boldsymbol{p})\right],
$$

where $\widehat{\Sigma}_{m}(\boldsymbol{p})$ denotes the surface mass density at the grid position $\left(X_{m}, Y_{m}\right)$ predicted by the model $\boldsymbol{p}$. For all clusters, we restrict the fitting to a square region of side $4 \mathrm{Mpc} h^{-1}$ centered on the cluster, where half the side length corresponds to the typical $r_{200 \mathrm{~m}}$ radius of CLASH clusters. This is to minimize the impact of the 2-halo term and local substructures that are abundant in cluster outskirts, which otherwise can lead to bias in cluster mass estimates (Meneghetti et al. 2010; Becker \& Kravtsov 2011; Rasia et al. 2012). Similarly, our previous CLASH studies performed fitting to azimuthally averaged lensing profiles by restricting the fitting range to $R \leqslant$ $2 \mathrm{Mpc}^{-1}$ (Umetsu et al. 2014; Merten et al. 2015; Umetsu et al. 2016).

We also fit the data with a spherical NFW halo $\left(q_{\perp}=1\right)$ using the same log-uniform priors on $M_{200 c}$ and $c_{200 c}$ in order to examine the consistency of our results with those of Umetsu et al. (2014).

In Table 1, we list the marginalized posterior constraints on each of the model parameters $\boldsymbol{p}=\left(M_{200 c}, c_{200 c}, q_{\perp}\right.$, PA) for 20 individual clusters of our sample. In this work, we employ the robust biweight estimators of Beers et al. (1990) for the center location $\left(C_{\mathrm{BI}}\right)$ and scale $\left(S_{\mathrm{BI}}\right)$ of the marginalized 1D posterior distributions (e.g., Stanford et al. 1998; Sereno \& Umetsu 2011; Biviano et al. 2013). The biweight estimator is insensitive to and stable against outliers, as it assigns higher weight to points that are closer to the center of the distribution (Beers et al. 1990). In the table, we also report, for each cluster, the minimum $\chi^{2}$ value per degree of freedom (dof) as an indicator of goodness of fit. Here, the number of dof is defined as the difference between the number of mass pixels within the fitting region and the number of free parameters. We find that the minimum $\chi^{2} /$ dof values for our sample range from 0.41

\footnotetext{
36 In principle, we can forward-model and directly fit a model to 2D shear and magnification constraints. This will require additional numerical integrals corresponding to the 2D Poisson equation (Keeton 2001).
}

(MACS J0744.9+3927) to 1.32 (RX J1347.5-1145), with a median of 0.80 . This indicates that complex morphologies in the projected cluster mass distribution (e.g., substructures and deviations from elliptical isodensity contours) are not statistically significant in individual clusters, and the eNFW model provides an adequate description of our $2 \mathrm{D}$ weak-lensing data.

In Appendix C, we show, for each of the clusters, the 2D marginalized posterior distributions of the eNFW parameters, with the contours enclosing $68 \%$ and $95 \%$ of the posterior probability (Figure 11). For each parameter, we also present the $1 \mathrm{D}$ marginalized distribution, in which the $C_{\mathrm{BI}}$ location is marked with a vertical line. We see that the mass and concentration parameters are well constrained by the data, except for MACS J1931.8-2635 and MACS J0429.6-0253, for which the posterior distribution on $c_{200 c}$ is largely informed by the prior, in the sense that the likelihood extends outside of the prior range. Overall, the $2 \mathrm{D}$ weak-lensing constraints on the halo shape parameters $\left(q_{\perp}, \mathrm{PA}\right)$ are not strongly degenerate with halo mass and concentration, as found by Oguri et al. (2010).

\section{Discussion}

\subsection{Systematic Errors}

We have accounted for various sources of errors associated with the weak-lensing shear and magnification measurements (Sections 3.3 and 3.4). All of these errors are encoded in the measurement uncertainties $\left(\sigma_{g}, \sigma_{\mu}\right)$ and the cosmic noise errors $\left(C_{g}^{\mathrm{lss}}, C_{\mu}^{\mathrm{lss}}\right)$, both of which contribute to the covariance matrix $C$ of the mass reconstruction (Appendix B).

Umetsu et al. (2016) quantified unaccounted sources of systematic errors in the CLASH weak-lensing measurements by considering the following effects: (1) dilution of the weaklensing signal by residual contamination from cluster members $(2.4 \% \pm 0.7 \%)$, (2) photo- $z$ bias in the mean depth estimates $(0.27 \%$; Section 3.2), and (3) shear calibration uncertainty (5\%; Section 3.3.2). These errors add up to $5.6 \%$ in quadrature, which is translated into the cluster mass uncertainty as $5.6 \% / \Gamma \simeq 7 \%$, with $\Gamma \simeq 0.75$ the typical value of the logarithmic derivative of the weak-lensing signal with respect to cluster mass (Melchior et al. 2017).

Alternatively, measuring the shear and magnification effects independently provides an empirical consistency check of weak-lensing measurements. Performing a shear-magnification consistency test, Umetsu et al. (2014) found the systematic uncertainty in the overall mass calibration to be $8 \%$. Following the CLASH program (Umetsu et al. 2014; Merten et al. 2015; Umetsu et al. 2016; Penna-Lima et al. 2017), we conservatively adopt this value as the systematic uncertainty in the ensemble mass calibration.

In Figure 2, we compare our mass estimates of 20 individual clusters from the present 2D weak-lensing analysis, $M_{\Delta \mathrm{c}}(2 \mathrm{D})$, with those from the 1D weak-lensing analysis of Umetsu et al. (2014), $M_{\Delta c}(1 D)$, shown for three characteristic overdensities. Since the two studies use the same data sets, this comparison allows us to assess the robustness and consistency of weaklensing mass determinations from different inversion methods. The mass estimates of Umetsu et al. (2014) were obtained assuming the spherical NFW profile, with the same priors on $M_{200 \mathrm{c}}$ and $c_{200 \mathrm{c}}$ as in Section 4.2. Results are shown separately for our NFW and eNFW mass estimates in the left and right 


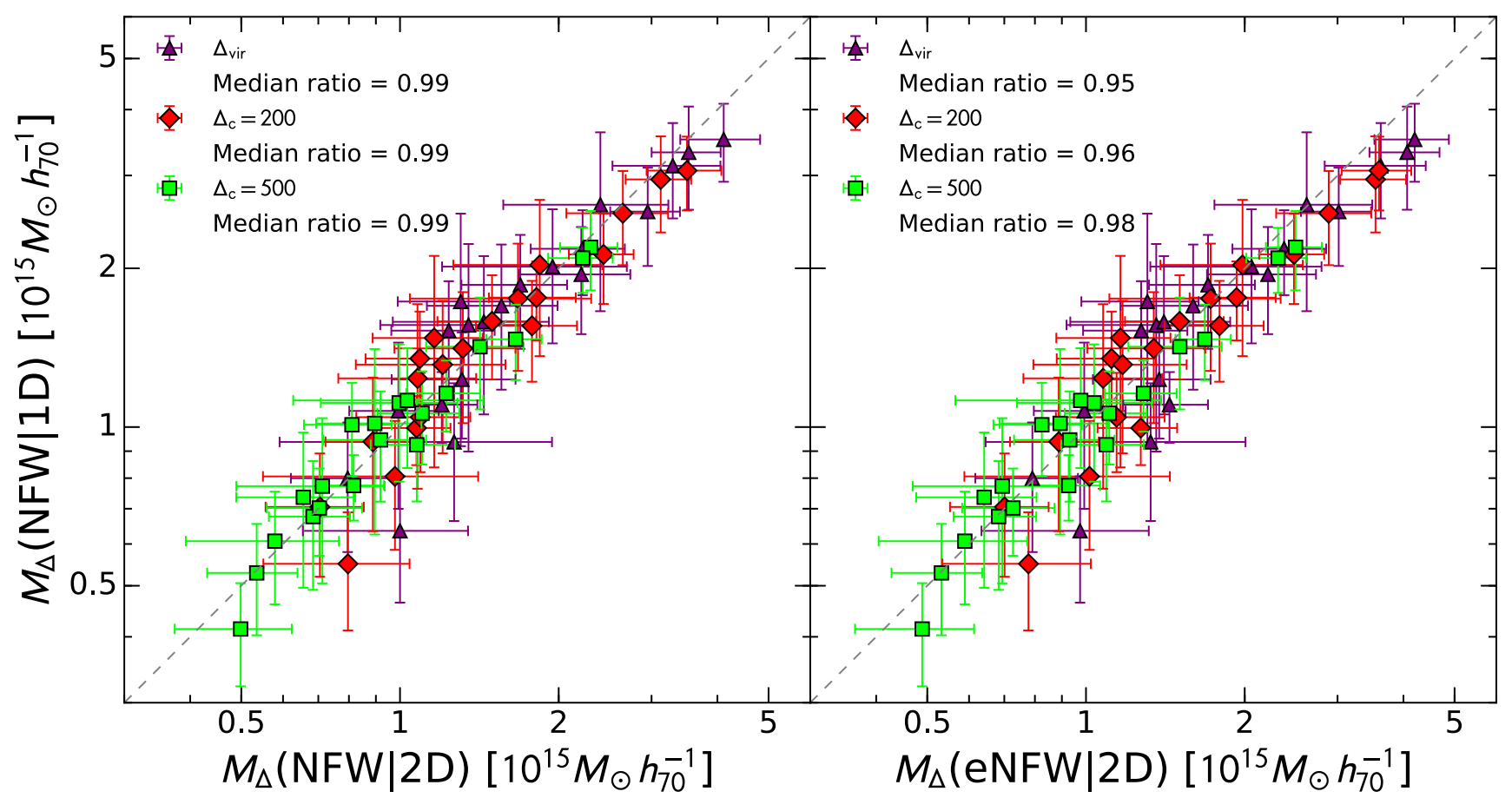

Figure 2. Comparison of weak-lensing mass estimates for our sample of 20 CLASH clusters derived from our full 2D analysis (horizontal axes) to those from the azimuthally averaged 1D analysis of Umetsu et al. (2014; vertical axis). The left and right panels present our 2D results obtained using the NFW and elliptical NFW (eNFW) density profiles, respectively. For each comparison, we measure the cluster mass for three characteristic overdensities, $\Delta_{\mathrm{vir}}$ (purple triangles), $\Delta_{\mathrm{c}}=200$ (red diamonds), and $\Delta_{\mathrm{c}}=500$ (green squares). The dashed line shows the one-to-one relation. The median mass ratios $\left\langle M_{\Delta}(1 \mathrm{D}) / M_{\Delta}(2 \mathrm{D})\right\rangle$ are reported in each panel.

panels, respectively. No aperture correction is applied in all cases.

As shown in the left panel of Figure 2, the $M_{\mathrm{vir}}, M_{200 \mathrm{c}}$, and $M_{500 \mathrm{c}}$ masses estimated from direct NFW fits to the 2D mass distribution $\Sigma(\boldsymbol{R})$ agree within $1 \%$ with those from the $\Sigma(R)$ profile of Umetsu et al. (2014). The right panel gives a comparison of our eNFW mass estimates with the NFW results of Umetsu et al. (2014), showing that the eNFW masses are on average $5 \%, 4 \%$, and $2 \%$ smaller for $\Delta_{\text {vir }}, \Delta_{\mathrm{c}}=200$, and $\Delta_{\mathrm{c}}=500$, respectively, than the NFW ones inferred from the azimuthally averaged 1D analysis. In summary, the mass estimates for our cluster sample derived with 1D and 2D inversion methods (Umetsu et al. 2014, 2016) agree well within the overall calibration uncertainty of $8 \%$, indicating that systematic effects due to azimuthal averaging applied to the shear data (Umetsu et al. 2014), as well as to details of the inversion procedures (Section 3.6), are not significant.

\subsection{Cluster Ellipticity}

\subsubsection{CLASH Ensemble Distribution}

We examine here the ensemble distribution of projected axis ratios using the results from the Bayesian inference of the eNFW parameters. Figure 3 shows the resulting distribution function for our sample of 20 clusters (blue squares), constructed from posterior point estimates $\left(C_{\mathrm{BI}}\right)$ of the $q_{\perp}$ parameter (Table 1). The median axis ratio for our sample, measured within a radial scale of $2 \mathrm{Mpc} h^{-1}$, is $\left\langle q_{\perp}\right\rangle=$ $0.67 \pm 0.07$, or $\langle\epsilon\rangle=0.33 \pm 0.07$ and $\langle e\rangle=0.38 \pm 0.08$ in terms of the projected halo ellipticity (Table 2), where the errors were estimated by bootstrap resampling the cluster sample. To check at which radius the constraint on the halo ellipticity effectively comes from, we calculate the massweighted, projected clustercentric radius $R_{\text {eff }}$ for our sample,

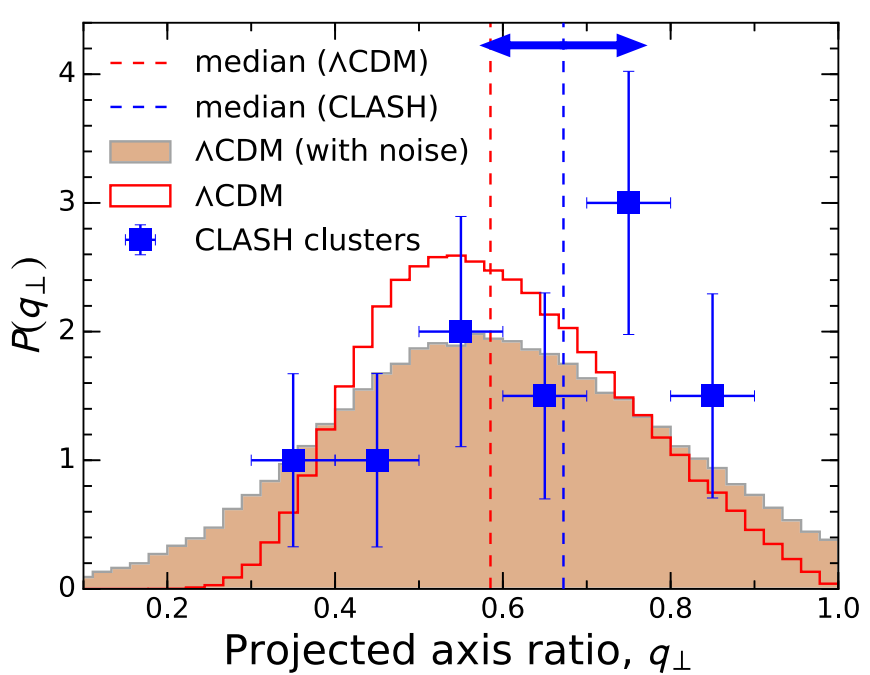

Figure 3. Distribution of projected axis ratios $q_{\perp}$ for our sample of $20 \mathrm{CLASH}$ clusters. The blue squares with error bars show the distribution constructed from point estimates of individual cluster posteriors (Table 1). The blue dashed vertical line corresponds to the median $\left\langle q_{\perp}\right\rangle=0.67 \pm 0.07$ of the distribution (Table 2), with the $1 \sigma$ error indicated by the double-headed arrow. The red shaded histogram represents the theoretical expectation based on the triaxial halo model of Bonamigo et al. (2015) assuming random orientations of the clusters, where the $q_{\perp}$ distribution predicted for each cluster has been convolved with a Gaussian of width equal to the observed uncertainty $\sigma\left(q_{\perp}\right)$. The red open histogram shows the theoretical distribution without the Gaussian convolution. The expected median value is $\left\langle q_{\perp}\right\rangle=0.59$ (red dashed vertical line) in both cases with and without the Gaussian convolution, consistent with our measurement at the $1 \sigma$ level.

averaged within the central $2 \mathrm{Mpc} h^{-1}$ region. Using the best-fit NFW model based on the stacked lensing analysis by Umetsu et al. (2016), we find $R_{\text {eff }} \simeq 0.89 \mathrm{Mpc} h^{-1}$. 
Table 2

Weak-lensing Halo Ellipticity Measurements

\begin{tabular}{lccc}
\hline \hline Sample & $N$ & $\langle\epsilon\rangle$ & $\langle e\rangle$ \\
\hline Full sample & 20 & $0.33 \pm 0.07$ & $0.38 \pm 0.08$ \\
X-ray selected & 16 & $0.28 \pm 0.07$ & $0.33 \pm 0.06$ \\
High-magnification & 4 & $0.45 \pm 0.11$ & $0.53 \pm 0.14$ \\
\hline
\end{tabular}

Note. Median values and $1 \sigma$ errors of weak-lensing cluster shape measurements derived for the full sample, the X-ray-selected subsample, and the highmagnification subsample. We adopt the halo ellipticity defined in two ways: $\epsilon=1-q_{\perp}$ and $e=\left(1-q_{\perp}^{2}\right) /\left(1+q_{\perp}^{2}\right)$ with $q_{\perp} \leqslant 1$ the projected halo axis ratio.

For a consistency check, we also create a composite probability distribution function (PDF) of $q_{\perp}$ by stacking the marginal posterior distributions (Figure 11) for all clusters in our sample. This yields a median axis ratio of $\left\langle q_{\perp}\right\rangle=$ $0.65 \pm 0.05$, or $\langle\epsilon\rangle=0.35 \pm 0.05$ and $\langle e\rangle=0.41 \pm 0.07$, where the errors are based on 50,000 random samples that are drawn from the posterior distributions for individual clusters. These results are in good agreement with those from the posterior point estimates.

It is important to note that the shape of the clusters in the CLASH sample is expected to be rounder on average due to a high fraction of relaxed clusters (Section 3.1; Meneghetti et al. 2014). Accordingly, there could be a bias toward higher values of the projected axis ratio in our sample. For our X-ray-selected subsample (excluding the four high-magnification clusters with complex morphologies), we find the median axis ratio to be $\left\langle q_{\perp}\right\rangle=0.72 \pm 0.07$ from the posterior point estimates and $\left\langle q_{\perp}\right\rangle=0.68 \pm 0.06$ from the stacked PDF. Although the amplitude of the shift is not statistically significant, the direction of the shift is consistent with the effect of the CLASH X-ray-selection function (Section 3.1).

Donahue et al. (2016) found the typical axis ratio for all 25 CLASH clusters in the Chandra X-ray brightness distribution to be $0.88 \pm 0.06$ within an aperture radius of $350 \mathrm{kpc} h^{-1}$ $\left(\sim 1.1 r_{2500 \mathrm{c}}\right)$, consistent with the value of $0.90 \pm 0.06$ inferred from the SZE maps observed with Bolocam operating at $140 \mathrm{GHz}$ (Sayers et al. 2013; Czakon et al. 2015). This comparison for the CLASH sample, albeit at different radial scales, indicates that the shape of the projected mass distribution as measured from weak lensing is more elongated than the gas distribution. This is qualitatively consistent with the theoretical expectation that the intracluster gas in hydrostatic equilibrium is rounder than the underlying matter distribution (Lee \& Suto 2003). However, we note that the values of cluster morphological parameters for the X-ray and SZE maps could be different if measured over larger radial scales (see the discussion in Section 5.2.2). On the other hand, our ground-based weak-lensing data do not sufficiently resolve morphological structures within a radial scale of $350 \mathrm{kpc} h^{-1}$ $(\sim 1 ! 6$ at the median sample redshift of $z=0.377)$ as they are limited by the small number density of background galaxies (Umetsu et al. 2014).

\subsubsection{Comparison with $\Lambda C D M$ Predictions}

These results can be compared with predictions from $\Lambda \mathrm{CDM}$ $N$-body simulations, in which DM halos are often modeled as triaxial ellipsoids (e.g., Jing \& Suto 2000; Bett et al. 2007; Despali et al. 2014; Bonamigo et al. 2015; Despali et al. 2016;
Vega-Ferrero et al. 2017). ${ }^{37}$ Here we restrict the comparison to predictions for the shape of the DM distribution measured within the virial radius, which is close to the maximum fitting radius of our analysis $\left(2 \mathrm{Mpc} h^{-1} \sim r_{200 \mathrm{~m}} \sim 1.1 r_{\text {vir }}\right)$. In particular, the triaxial model of Bonamigo et al. (2015) is of particular interest because they extended the original work of Jing \& Suto (2000) to a wider mass range with higher precision. Bonamigo et al. (2015) selected and analyzed those halos for which the offset between the center of mass and geometrical center of the ellipsoid is less than $5 \%$ of their virial radius. The fraction of selected clusters is $\sim 50 \%$ at $z=0$ (see Figure 2 of Bonamigo et al. 2015). On the other hand, applying stringent relaxation selection criteria typically results in a much smaller fraction of selected halos (e.g., $\sim 15 \%$ at $z=0.25$ as found by Meneghetti et al. 2014).

In Figure 3, we show the theoretical expectation $P\left(q_{\perp}\right)$ for our composite clusters (red shaded histogram) obtained using the fitting formula of Bonamigo et al. (2015), which describes the intrinsic distribution of triaxial axis ratios for their DM halos. Here, the predicted distribution has been constructed as follows: first, we compute for each cluster the intrinsic axis ratio distribution as a function of halo virial mass $M_{\text {vir }}$ and redshift (Bonamigo et al. 2015) by accounting for the uncertainty in the mass determination. Next, we construct the distribution of projected axis ratios by projecting triaxial halos onto the sky plane assuming random orientations. The $q_{\perp}$ distribution predicted for each cluster is then convolved with a Gaussian of width equal to the observed uncertainty $\sigma\left(q_{\perp}\right)$. The composite PDF is finally obtained by adding the renormalized distributions of all 20 clusters. In Figure 3, we also show the theoretical distribution $P\left(q_{\perp}\right)$ without the Gaussian convolution (red open histogram). Because of the projection effect, the overall shape of the distribution of the projected axis ratio is broader and shifted to values (rounder) higher than those of the intrinsic minor-to-major axis ratio (Suto et al. 2016). The expected median value is $\left\langle q_{\perp}\right\rangle=0.59$ in both cases with and without the Gaussian convolution, in agreement with our measurement within the uncertainty.We reiterate that our sample is expected to contain a high fraction of relaxed clusters $(\sim 60 \%$ in our full composite sample, compared to $\sim 70 \%$ in the X-ray-selected subsample; see Section 3.1), and hence the average projected axis ratio is likely biased high to some degree. In particular, if we restrict our comparison to the $\mathrm{X}$-ray-selected subsample, the observed median projected axis ratio (Table 2) is higher at the $1.8 \sigma$ level than predicted from the triaxial model of Bonamigo et al. (2015).

We plot in Figure 4 the projected axis ratio $q_{\perp}$ as a function of the virial mass $M_{\text {vir }}$ for our sample of 20 CLASH clusters (black squares). The blue triangle in Figure 4 represents the (unweighted) median values for the sample, $\left\langle q_{\perp}\right\rangle=0.67 \pm$ 0.07 at $\left\langle M_{\mathrm{vir}}\right\rangle=(15.2 \pm 2.8) \times 10^{14} M_{\odot} h_{70}^{-1}$. Again, our CLASH weak-lensing measurements are in good agreement with $\Lambda$ CDM expectations at the median sample redshift of $z=0.377$. All 20 CLASH clusters lie within the $2 \sigma$ distribution predicted with the triaxial model of Bonamigo et al. (2015) assuming random orientations of the clusters.

Suto et al. (2016) studied the mass and radial dependence and the redshift evolution of the non-sphericity of cluster-size halos using DM-only simulations. They found that the average $3 \mathrm{D}$ minor-to-major axis ratio of simulated halos has a strong

\footnotetext{
37 A triaxial halo in projection is seen as elliptical isodensity contours (Stark 1977).
} 


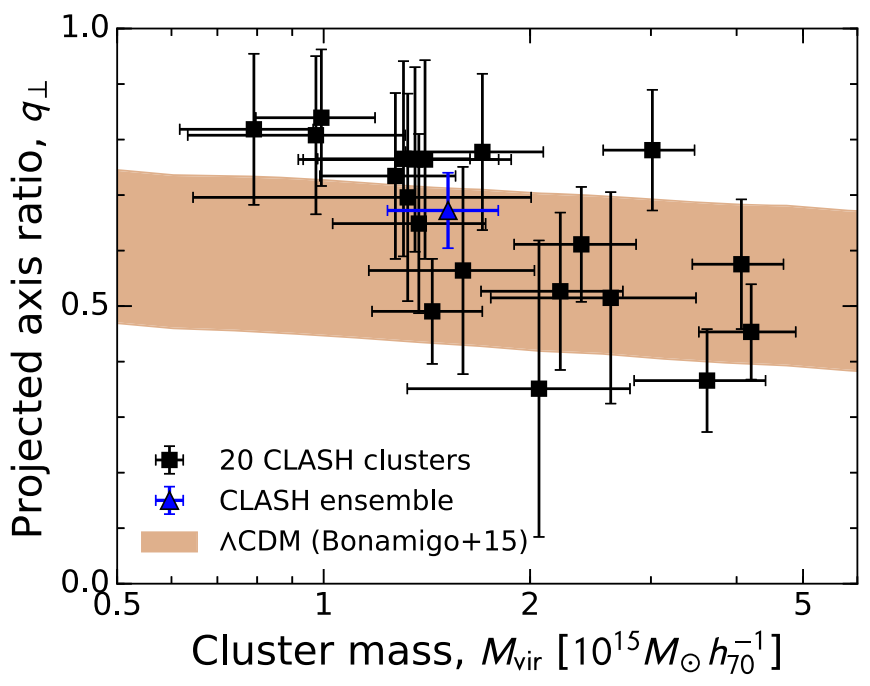

Figure 4. Projected halo axis ratio $q_{\perp}$ plotted against the virial mass $M_{\mathrm{vir}}$ for our sample of $20 \mathrm{CLASH}$ clusters (black squares). The blue triangle represents the median values for the sample, $\left\langle q_{\perp}\right\rangle=0.67 \pm 0.07$ at $\left\langle M_{\mathrm{vir}}\right\rangle=(15.2 \pm$ 2.8) $\times 10^{14} M_{\odot} h_{70}^{-1}$. The red shaded region represents the $1 \sigma$ range of the theoretical distribution at $z=0.377$ predicted with the triaxial model of Bonamigo et al. (2015) assuming random orientations of the clusters.

radial dependence as a function of enclosed mass, indicating that the internal structure of halos deviates from a self-similar geometry of concentric ellipsoidal surfaces. Suto et al. (2016) thus constructed the PDF of the projected axis ratio, $P\left(q_{\perp}\right)$, directly from the projected density distributions of simulated halos, without involving triaxial modeling. ${ }^{38}$ Using their fitting formula for the PDF at $z=0.4$, the mean, standard deviation, and median are found to be $0.57,0.17$, and 0.58 , respectively. The predicted median agrees with our full-sample result at the $1.3 \sigma$ level. On the other hand, when halos in multicomponent systems are excluded from their analysis, the mean, standard deviation, and median of the PDF are 0.59, 0.16, and 0.60, respectively. The observed median axis ratio for the CLASH $\mathrm{X}$-ray-selected subsample is $1.6 \sigma$ above this predicted median.

More recently, it was shown by Suto et al. (2017) that baryonic physics operating in the cluster central region, such as cooling and feedback effects, can have a substantial impact on the non-sphericity of cluster halos up to half the virial radius, even though these baryonic effects have little impact on the spherically averaged DM density profile. They found that the DM distribution becomes more spherical, depending on the distance from the cluster center, when the effects of baryons are included. Since our sample comprises highly relaxed and highly disturbed clusters (Section 3.1), a more quantitative comparison with theoretical expectations would require a detailed modeling of baryonic physics by accounting for the selection function. In fact, Figure 4 shows a slight tendency for lower-mass clusters to have projected axis ratios that are higher than the predicted distribution. This tendency is qualitatively consistent with the combination of the CLASH selection function and the baryonic effects. Nevertheless, our analysis is currently limited by the small number of clusters.

\footnotetext{
${ }^{38}$ We note that although their typical halo mass $\left(M_{\text {vir }}=2 \times 10^{14} M_{\odot} h^{-1}\right.$ at $z=0.2)$ is considerably smaller than that of our sample, $M_{\mathrm{vir}} \sim 11 \times 10^{14}$ $M_{\odot} h^{-1}$, this is not critical because the mean projected axis ratio exhibits little mass dependence over the relevant mass interval (Figure 11 of Suto et al. 2016).
}

\subsubsection{Comparison with Other Observational Studies}

Our CLASH lensing results can be compared to the weaklensing measurements obtained by the LoCuSS collaboration (Oguri et al. 2010), who performed a 2D shear-fitting analysis on a sample of $25 \mathrm{X}$-ray-luminous clusters at $\langle z\rangle \sim 0.2$. Oguri et al. (2010) modeled the projected mass distribution in each individual cluster assuming a single eNFW halo, as done in our work. They fitted an eNFW profile to the grid-averaged 2D reduced shear field. For a subset of 18 clusters that were specifically chosen to give good ellipticity constraints, they found $\mathrm{a} \sim 7 \sigma$ detection of the mean cluster ellipticity, $\langle\epsilon\rangle=0.46 \pm 0.04$, for their clusters with $\left\langle M_{\mathrm{vir}}\right\rangle \sim 10 \times$ $10^{14} M_{\odot} h_{70}^{-1}$. Their mean ellipticity is higher than, but consistent within the errors with, our full-sample measurement (Table 2). This difference is not statistically significant, but could be due in part to the CLASH X-ray selection function (Section 3.1).

Similarly, Oguri et al. (2012) found from their 2D shear analysis of 25 strong-lensing-selected clusters that the stacked cluster ellipticity is nearly constant, $\langle\epsilon\rangle \sim 0.45$, with cluster radius within their errors.

On group/cluster scales, several authors have constrained the halo ellipticity from stacked weak-lensing measurements (Evans \& Bridle 2009; Clampitt \& Jain 2016; van Uitert et al. 2017; Shin et al. 2018) using quadrupole shear estimators and their variants (e.g., Natarajan \& Refregier 2000; Adhikari et al. 2015; Clampitt \& Jain 2016). Their stacked weak-lensing measurements span a range of mean $q_{\perp}$ values from $0.48_{-0.09}^{+0.14}$ (Evans \& Bridle 2009) to $\sim 0.78$ (Clampitt \& Jain 2016), broadly consistent with $\Lambda$ CDM predictions. We note that, in their approach, one probes halo quadrupoles with respect to the major axis of the light distribution (e.g., BCGs) chosen as a reference orientation. On the other hand, we have directly measured the shape and orientation of individual cluster halos from deep high-quality weak-lensing data (Umetsu et al. 2014). We present in Section 5.3.2 our stacked quadrupole shear measurement for our full sample of 20 CLASH clusters using the shape of the Chandra X-ray brightness distribution (see Section 5.3.1) as a reference orientation.

\subsection{Cluster Misalignment Statistics \\ 5.3.1. Alignments of CLASH Clusters}

Donahue et al. $(2015,2016)$ presented a detailed study of morphologies and alignments of BCGs, intracluster gas, and mass at small cluster radii for the CLASH sample. Donahue et al. (2015) measured PAs for all 20 X-ray-selected CLASH clusters from the rest-frame ultraviolet (UV) and near-infrared (NIR) light distributions of the BCGs using CLASH HST data (see their Table 3). Similarly, Donahue et al. (2016) examined the morphological properties of all 25 CLASH clusters with Chandra X-ray data (Donahue et al. 2014) and compared them with those inferred from Bolocam SZE maps of the intracluster gas (Sayers et al. 2013; Czakon et al. 2015) and those from mass maps based on HST strong and weak lensing (hereafter HST-GL; Zitrin et al. 2015). The lensing maps used are based on parametric modeling assuming that light traces mass for the galaxy-scale mass components (see Meneghetti et al. 2017). They found a strong correlation between PAs as measured from the X-ray, SZE, and HST-GL maps inside a consistent metric aperture of radius $350 \mathrm{kpc} h^{-1}\left(\sim 0.2 r_{\text {vir }}\right.$ for these clusters). 
They also found a strong alignment of the cluster shapes at this scale, as measured from the X-ray, SZE, and HST-GL maps, with that of the NIR BCG light at $10 \mathrm{kpc}$ scales. In particular, they obtained a median misalignment angle of $|\Delta \mathrm{PA}| \simeq 11^{\circ}$ between the BCG and X-ray orientations for the 20 X-rayselected CLASH clusters.

Now we compare the PAs determined from our 2D weaklensing analysis (WL) to those from three baryonic tracers, namely the BCGs, X-ray maps, and SZE maps, as well as from the HST-GL maps. To this end, we use the BCG PAs derived from the HST NIR images (Table 3 of Donahue et al. 2015), and the X-ray, SZE, and HST-GL PAs measured within an aperture radius of $350 \mathrm{kpc} h^{-1}$ (Tables 3, 6, and 5 of Donahue et al. 2016). To be conservative, we estimate errors on the BCG PAs from differences between the HST UV and NIR measurements of Donahue et al. (2015). There are 16, 20, 18 , and 20 clusters available for BCG/WL, X-ray/WL, SZE/ WL, and HST-GL/WL comparisons, respectively. The typical uncertainty in the weak-lensing PA measurements is $\sim 20^{\circ}$ per cluster, comparable to that in the SZE measurements, while those in the BCG, X-ray, and HST-GL measurements are much smaller, $\sim 10^{\circ}, \sim 4^{\circ}$, and $\sim 4^{\circ}$ per cluster, respectively.

In Figure 5, the PAs of the clusters determined from our weak-lensing analysis are plotted against those from the BCGs, $\mathrm{X}$-ray maps, SZE maps, and HST-GL maps. To quantify the degree of correlation between PAs of different tracers, we calculate the Spearman rank correlation coefficient $r_{P}$ and the corresponding probability $(p)$ for the null hypothesis of random orientations. The test indicates a similarly good correlation in all four of these comparisons, where a low probability indicates high significance of correlation: $r_{\mathrm{S}}=0.69\left(p=2.9 \times 10^{-3}\right)$ for $\mathrm{BCG} / \mathrm{WL}, r_{\mathrm{S}}=0.66\left(p=1.7 \times 10^{-3}\right)$ for $\mathrm{X}$-ray $/ \mathrm{WL}$, $r_{\mathrm{S}}=0.58\left(p=1.1 \times 10^{-2}\right)$ for SZE/WL PAs, and $r_{\mathrm{S}}=0.74$ $\left(p=1.7 \times 10^{-4}\right)$ for HST-GL/WL PAs.

Next, we quantify the alignment of the different components in the clusters by constructing the probability distributions of the absolute misalignment angles $|\triangle \mathrm{PA}|$ (e.g., Faltenbacher et al. 2009; Zhang et al. 2013; West et al. 2017) with respect to the weak-lensing halo shape. In the absence of any alignment, $|\triangle \mathrm{PA}|$ follows a uniform distribution between $0^{\circ}$ and $90^{\circ}$, with a mean (median) of $\langle|\Delta \mathrm{PA}|\rangle=45^{\circ}$. Values of $\langle|\Delta \mathrm{PA}|\rangle<45^{\circ}$ indicate that the PAs of the two distributions are, on average, aligned parallel with each other.

We show in Figure 6 the histogram distributions of $|\Delta \mathrm{PA}|$ between the BCG/WL, X-ray/WL, SZE/WL, and HST-GL/ WL orientations derived for our sample. Table 3 lists, for the respective comparisons, the median values of $|\Delta \mathrm{PA}|$ and the corresponding significance probabilities based on the posterior point estimates of weak-lensing PAs. As evident from Table 3 and Figure 6, among the three baryonic tracers studied here, the $\mathrm{X}$-ray morphology is best aligned with the weak-lensing mass distribution. For this comparison, we find a median misalignment angle of $\langle|\Delta \mathrm{PA}|\rangle=21^{\circ} \pm 7^{\circ}$ (Table 3), corresponding to $3.6 \sigma$ significance with respect to the null hypothesis of random orientations. However, these median values are biased high relative to their intrinsic values because they are estimated from the noisy distributions. If the intrinsic PA difference $\triangle \mathrm{PA}=$ PA(X-ray) - PA(WL) follows a (truncated) Gaussian distribution with a zero mean and a dispersion $\sigma_{\text {int }}$, the median of the intrinsic distribution of $|\Delta \mathrm{PA}|$ is $\simeq \sigma_{\text {int }} / 1.483$. In the presence of noise, the apparent dispersion from observations is increased. We correct for the effect of this noise bias assuming that the PA errors follow a Gaussian distribution. Adopting the typical uncertainties in the PA measurements, we find the bias-corrected median misalignment angle to be $\langle|\Delta \mathrm{PA}|\rangle=16^{\circ} \pm 7^{\circ}$.

For the BCG/WL and SZE/WL comparisons, we find weaker alignment signals $\left(<45^{\circ}\right)$, which are consistent with a null detection within the errors. A weak constraint on the SZE/ WL alignment is in line with expectations accounting for the large errors in both measurements. On the other hand, the weak signal in the BCG/WL alignment appears to be contradictory to the high degree of correlation found between the BCG/WL PAs (Figure 5). ${ }^{39}$ This is largely because the distribution of $\triangle \mathrm{PA}=\mathrm{PA}(\mathrm{BCG})-\mathrm{PA}(\mathrm{WL})$ is not symmetric about $\triangle \mathrm{PA}=$ $0^{\circ}$ (Figure 5), with a median offset of $\langle\Delta \mathrm{PA}\rangle=-25^{\circ} \pm 14^{\circ}$. For the X-ray/WL, SZE/WL, and HST-GL/WL comparisons, the median offsets are found to be $\langle\Delta \mathrm{PA}\rangle=-3^{\circ} \pm 9^{\circ}$, $7^{\circ} \pm 17^{\circ}$, and $-12^{\circ} \pm 6^{\circ}$, respectively (Table 3 ).

On the other hand, a strong alignment is found between the WL and HST-GL shapes at different radial scales, with a median misalignment angle of $\langle|\Delta \mathrm{PA}|\rangle=16^{\circ} \pm 4^{\circ}$. This corresponds to a significance level of $7.7 \sigma$ with respect to the null hypothesis. Applying the noise-bias correction yields $\langle|\Delta \mathrm{PA}|\rangle=8^{\circ} \pm 4^{\circ}$. This is consistent with the results of Despali et al. (2016), who found from $N$-body simulations that, for cluster-scale halos, the innermost and outermost mass ellipsoids are aligned with each other within $10^{\circ}$. We emphasize that this strong alignment signal has been found despite using two independent data sets (HST versus groundbased observations) and substantially different modeling methods.

\subsubsection{Weak-lensing Quadrupole Shear Measurement}

Here we present a complementary quadrupole shear analysis to test the consistency of our cluster ellipticity measurements for our sample of $20 \mathrm{CLASH}$ clusters. To this end, we employ the Cartesian estimators of Clampitt \& Jain (2016) that null the purely tangential, monopole shear contribution. Specifically, we measure the stacked quadrupole shear signal with respect to a coordinate system with the $x$ axis aligned with the X-ray major axis of each cluster. We adopt the same sign convention for the Cartesian $g_{1}$ and $g_{2}$ components as defined in Clampitt \& Jain (2016; see their Figure 1) and use $\theta$ to denote the azimuthal angle relative to the $x$-axis. Following Clampitt \& Jain (2016), we group together the first and second shear components of background galaxies in the regions where $\cos 4 \theta$ and $\sin 4 \theta$ have the same sign (Figure 7), respectively, and define the following estimator:

$$
\Delta \Sigma_{\alpha}^{(s)}(R)=\Sigma_{\mathrm{c}}\left[\sum_{k} w_{(k)} g_{\alpha, k}\right]\left[\sum_{k} w_{(k)}\right]^{-1},
$$

where we have introduced the notation in analogy to the tangential shear, which probes the differential surface mass density $\Delta \Sigma$ (e.g., Umetsu et al. 2014). Here, $\Sigma_{\mathrm{c}} \propto\langle\beta\rangle_{g}^{-1} D_{1}^{-1}$ is the source-averaged critical surface density for a given cluster (Section 2.1), $w_{(k)}$ is the statistical weight for the $k$ th background galaxy (Section 3.3.1), and $k$ runs over all background galaxies that fall in the specified bin, different for each shear component $\alpha$ and sign $s$ (Clampitt \& Jain 2016):

\footnotetext{
39 Similar results are found when the Spearman's rank correlation coefficient is used instead of $r_{P}$, as it is invariant under constant shifts.
} 

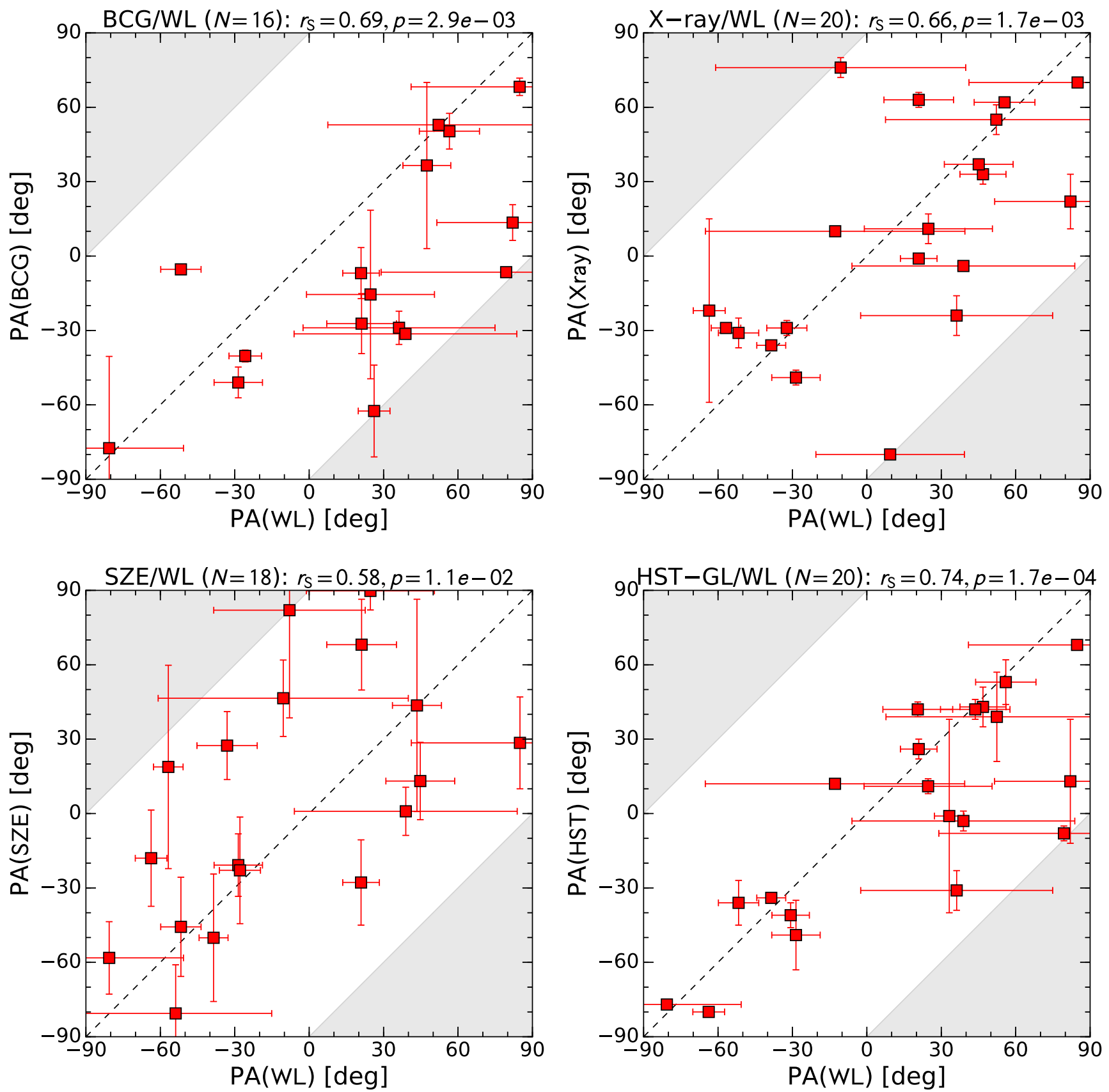

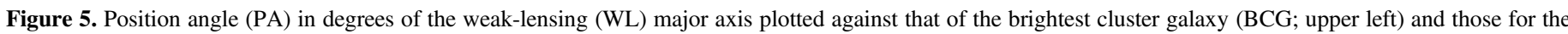

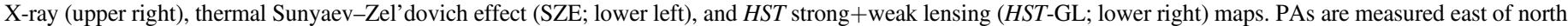

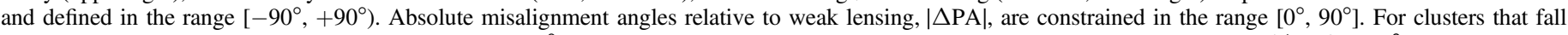

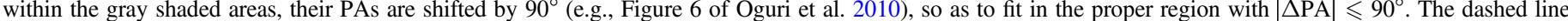

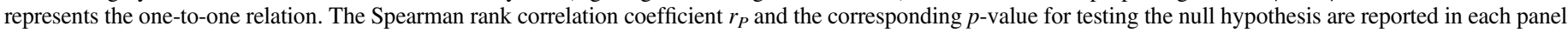

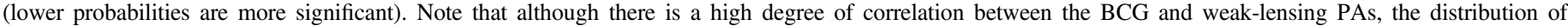
$\Delta \mathrm{PA}=\mathrm{PA}(\mathrm{BCG})-\mathrm{PA}(\mathrm{WL})$ is not symmetric about $\Delta \mathrm{PA}=0^{\circ}$, with a median offset of $\langle\Delta \mathrm{PA}\rangle=-25^{\circ} \pm 14^{\circ}(\mathrm{Table} 3)$.

$\alpha=1, s=-,-\pi / 8 \leqslant \theta_{j}<\pi / 8 ; \alpha=1, s=+, \pi / 8 \leqslant \theta_{j}$ $<3 \pi / 8 ; \quad \alpha=2, \quad s=-, \quad 0 \leqslant \theta_{j}<\pi / 4 ; \quad \alpha=2, \quad s=+$, $\pi / 4 \leqslant \theta_{j}<\pi / 2$. For each case, the summation in Equation (31) also includes background galaxies lying in symmetrical regions shifted by $\pi / 2, \pi$, and $3 \pi / 2$, as illustrated in Figure 7.

In this work, we first measure for each cluster the quadrupole shear profiles $\Delta \Sigma_{\alpha}^{(s)}(R)$ from the background-selected shear catalog according to Equation (31), and then stack all clusters together by

$$
\begin{aligned}
\left\langle\left\langle\Delta \Sigma_{\alpha}^{(s)}(R)\right\rangle\right\rangle & =\left[\sum_{n} W_{n} \Delta \Sigma_{\alpha, n}^{(s)}(R)\right]\left[\sum_{n} W_{n}\right]^{-1}, \\
W_{n} & =1 /\left[\sigma_{\alpha, n}^{(s)}(R)\right]^{2},
\end{aligned}
$$

where $\langle\langle\ldots\rangle\rangle$ denotes the sensitivity-weighted average over the cluster sample (Umetsu et al. 2014, 2016), $n$ runs over all 20 

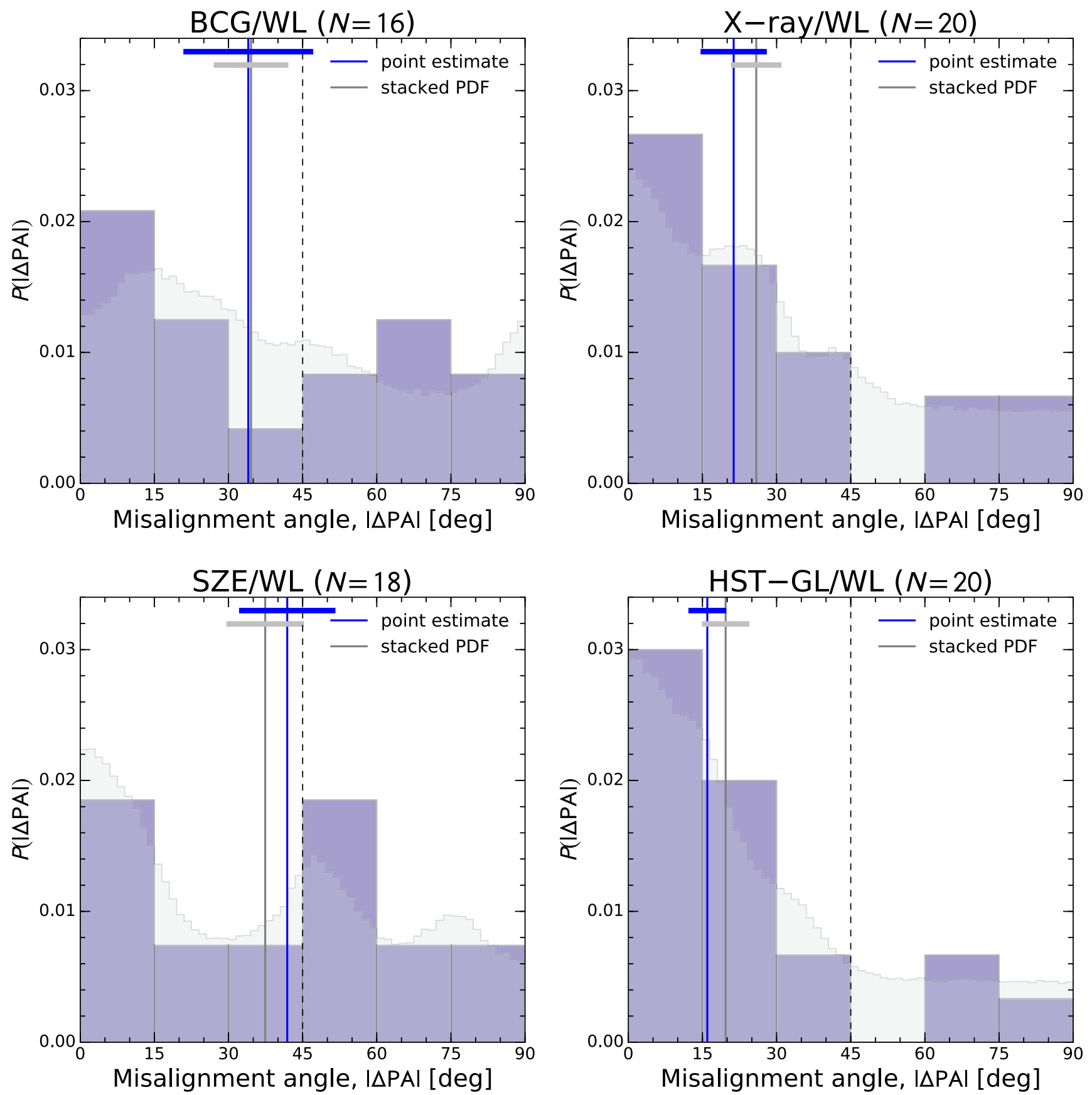

Figure 6. Histogram of the absolute misalignment angles $|\triangle \mathrm{PA}|$ between the BCG/WL (upper left), X-ray/WL (upper right), SZE/WL (lower left), and $H S T$-GL/WL (lower right) major axes. In each panel, the blue and gray shaded histograms show the distributions constructed from posterior point estimates $\left(C_{\mathrm{BI}}\right)$ and from the stacked composite PDF, respectively. For each histogram, the median value of the distribution is indicated by a vertical line, and its $1 \sigma$ error by a thick horizontal line. In the absence of any alignment, the expected median value is $45^{\circ}$ (vertical dashed line).

clusters in our sample, and $\sigma_{\alpha, n}^{(s)}(R)$ is the uncertainty of $\Delta \Sigma_{\alpha, n}^{(s)}(R)$ estimated from bootstrap resampling of the background galaxies. We estimate the errors of the stacked $\left\langle\left\langle\Delta \Sigma_{\alpha}^{(s)}(R)\right\rangle\right\rangle$ profiles by bootstrap resampling the cluster sample. The resulting stacked quadrupole profiles for our cluster sample are shown in Figure 8.

Clampitt \& Jain (2016) modeled the quadrupole shear signal using a multipole expansion of the surface mass density of elliptical halos (Adhikari et al. 2015). However, this method can only be applied to the case with a small halo ellipticity, so that the higher-order terms can be safely ignored.
In order to accurately model the observed signal and to make a direct comparison with our 2D cluster ellipticity measurements (Section 5.2), we forward-model the stacked quadrupole shear profiles by assuming an eNFW halo with the major axis aligned with the $\mathrm{X}$-ray major axis, that is, $\triangle \mathrm{PA}=\mathrm{PA}(\mathrm{X}$-ray $)-\mathrm{PA}$ $(\mathrm{WL})=0^{\circ}$. Therefore, any misalignment $|\Delta \mathrm{PA}|>0^{\circ}$ will lead to dilution of the quadrupole signal and hence underestimation of the halo ellipticity. We use a Bayesian MCMC approach (see Section 4.2.2) to simultaneously fit an eNFW model to the four stacked quadrupole profiles, namely $\left\langle\left\langle\Delta \Sigma_{1}^{(-)}\right\rangle\right\rangle,\left\langle\left\langle\Delta \Sigma_{1}^{(+)}\right\rangle\right\rangle$, $\left\langle\left\langle\Delta \Sigma_{2}^{(-)}\right\rangle\right\rangle$, and $\left\langle\left\langle\Delta \Sigma_{2}^{(+)}\right\rangle\right\rangle$, each measured in four radial bins 
Table 3

Weak-lensing Halo Misalignment Statistics

\begin{tabular}{lcclcc}
\hline \hline \multicolumn{1}{c}{ Data Sets } & $N$ & $\begin{array}{c}\langle\Delta \mathrm{PA}\rangle \\
\text { (degrees) }\end{array}$ & $\begin{array}{c}\langle|\Delta \mathrm{PA}|\rangle \\
\text { (degrees) }\end{array}$ & $p$-value & $p_{\text {Bin }}$ \\
\hline $\mathrm{BCG} / \mathrm{WL}$ & 16 & $-25 \pm 14$ & $34 \pm 13$ & $1.5 \times 10^{-1}$ & $1.8 \times 10^{-1}$ \\
$\mathrm{X}-\mathrm{ray} / \mathrm{WL}$ & 20 & $-3 \pm 9$ & $21 \pm 7$ & $4.1 \times 10^{-3}$ & $4.6 \times 10^{-3}$ \\
$\mathrm{SZE} / \mathrm{WL}$ & 18 & $7 \pm 17$ & $42 \pm 10$ & $3.8 \times 10^{-1}$ & $1.9 \times 10^{-1}$ \\
$\mathrm{HST}$-GL/WL & 20 & $-12 \pm 6$ & $16 \pm 4$ & $3.1 \times 10^{-4}$ & $1.1 \times 10^{-3}$
\end{tabular}

Note. The basic statistics of misalignment angles are listed. The results here are based on the point estimates of weak-lensing PAs from individual cluster posterior distributions (Figure 11). Column 1: combination of data sets. Column 2: number of clusters in the overlapping sample. Column 3: median of the distribution of misalignment angles, $\triangle \mathrm{PA} \in\left[-90^{\circ}, 90^{\circ}\right)$. Column 4 : median of the distribution of absolute misalignment angles, $|\Delta \mathrm{PA}| \in\left[0^{\circ}, 90^{\circ}\right]$. Column 5: probability of finding the value $\langle|\Delta \mathrm{PA}|\rangle$ smaller than or equal to the observed value when the null hypothesis of a uniform distribution in $\left[0^{\circ}, 90^{\circ}\right]$ is true. Column 6: probability, obtained with the binomial test, that the observed distribution in $|\triangle \mathrm{PA}|$ has random orientations (West et al. 2017).
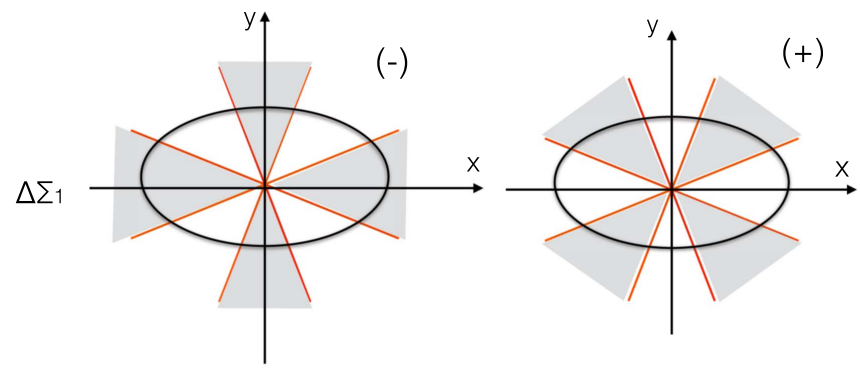

$\Delta \Sigma 2$
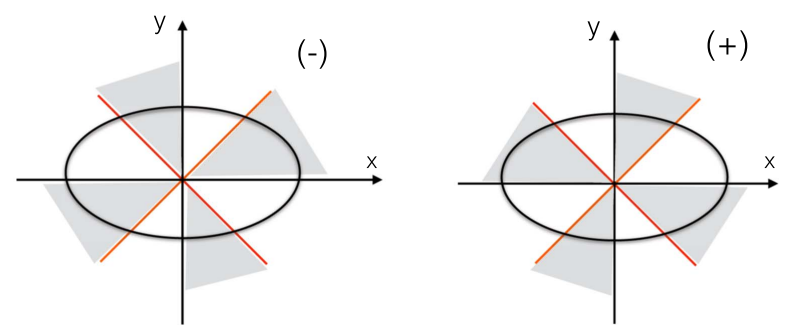

Figure 7. Illustration of the Cartesian quadrupole shear estimators of Clampitt \& Jain (2016). The $x$-axis of the Cartesian coordinate system is aligned with the major axis of the X-ray brightness distribution, assumed to be aligned with the major axis of the underlying mass distribution. We group together the Cartesian first and second shear components in same-sign regions of $\cos 4 \theta$ and $\sin 4 \theta$ (gray shaded regions), respectively, and define four quadrupole shear components, namely, $\Delta \Sigma_{1}^{(-)}$(upper left), $\Delta \Sigma_{1}^{(+)}$(upper right), $\Delta \Sigma_{2}^{(-)}$(lower left), and $\Delta \Sigma_{2}^{(+)}$(lower right).

(Figure 8). We use a uniform prior distribution for the projected axis ratio in the range $0.1 \leqslant q_{\perp} \leqslant 1$ (Section 5.2). We marginalize over the mass and concentration parameters using Gaussian priors of $M_{200 \mathrm{c}}=(10.9 \pm 0.7) \times 10^{14} M_{\odot} h^{-1}$ and $c_{200 \mathrm{c}}=3.3 \pm 0.2$ based on the joint strong-lensing, weaklensing shear and magnification analysis of Umetsu et al. (2016). Marginalized posterior constraints $\left(C_{\mathrm{BI}} \pm \mathrm{S}_{\mathrm{BI}}\right)$ on the projected axis ratio are obtained as $q_{\perp}=0.67 \pm 0.10$ (Figure 8), or $\epsilon=0.33 \pm 0.10$ and $e=0.38 \pm 0.12$ in terms of the halo ellipticity. These are in excellent agreement with the results from the $2 \mathrm{D}$ weak-lensing analysis of 20 individual clusters (Table 2), supporting the robustness of our results and indicating that the effect of dilution due to X-ray/WL misalignment is not significant for our cluster sample.

\subsubsection{Comparison with Previous Cluster-scale Alignment Studies}

Okumura et al. (2009) measured intrinsic ellipticity correlation functions for a large sample of luminous red galaxies (LRGs) in the redshift range 0.16-0.47 selected from Data Release 6 (DR6) of the Sloan Digital Sky Survey (SDSS), finding a clear signal up to a scale of $30 \mathrm{Mpc}^{-1}$. To model the observed ellipticity correlation, they populated galaxies into DM halos in cosmological $N$-body simulations using a halo occupation distribution approach. In this context, the fraction of synthetic central LRGs is $93.7 \%$, and they are hosted by cluster-scale DM halos with $M>8 \times 10^{13} M_{\odot}$. The ellipticity correlation is predicted to have an amplitude that is about four times higher than their measurement when assuming a perfect alignment between the central LRGs and their host DM halos. Assuming a Gaussian misalignment between the major axes of central LRGs and host halos, they found a misalignment dispersion of $\sigma(\Delta \mathrm{PA})=35.4_{-3.3}^{+4.0}$ degrees, or an absolute median of $\langle|\Delta \mathrm{PA}|\rangle=24_{-2}^{+3}$ degrees. A similar constraint was derived by Okumura \& Jing (2009) from the gravitational shear-intrinsic ellipticity correlation function measured using the LRG sample. Their constraints on the misalignment angle are in agreement with our BCG/WL results within the errors (Table 3).

Huang et al. (2016) studied central galaxy alignments with respect to the spatial distribution of member galaxies using the red-sequence Matched-filter Probabilistic Percolation (redMaPPer) cluster catalog based on the SDSS DR8, finding an absolute mean misalignment angle of $\sim 35^{\circ}$, corresponding to an absolute median angle of $\langle|\Delta \mathrm{PA}|\rangle \sim 30^{\circ}$. This is in agreement with our estimate for the median misalignment angle between the BCG/WL major axes (Table 3) if assuming that cluster member galaxies are an unbiased probe of the underlying mass distribution.

van Uitert et al. (2017) presented a stacked quadrupole shear analysis of 2600 galaxy groups selected from the Galaxy And Mass Assembly survey using the galaxy shear catalog from the Kilo Degree Survey. On small scales $(<250 \mathrm{kpc})$, they found the major axis of the $\mathrm{BCG}$ to be the best proxy of the orientation of the underlying mass distribution. On larger scales, a much weaker correlation was found between the orientations of the BCG and the mass distribution, while the distribution of member galaxies provides a better proxy for the orientation of the overall mass distribution in groups.

More recently, Shin et al. (2018) studied the stacked halo ellipticity for a sample of $\sim 10^{4}$ SDSS redMaPPer clusters. Stacking the quadrupole shear signal along the major axis of the cluster member distribution, they found a mean axis ratio of $0.558 \pm 0.086 \pm 0.026$ (statistical followed by systematic uncertainty). This agrees well with the mean axis ratio of the member distribution, $0.573 \pm 0.002 \pm 0.039$, indicating that cluster galaxies trace the shape of the cluster mass distribution within their errors. On the other hand, they found an rms misalignment angle of $30^{\circ} \pm 10^{\circ}$ between the central galaxy and the cluster mass distribution. 

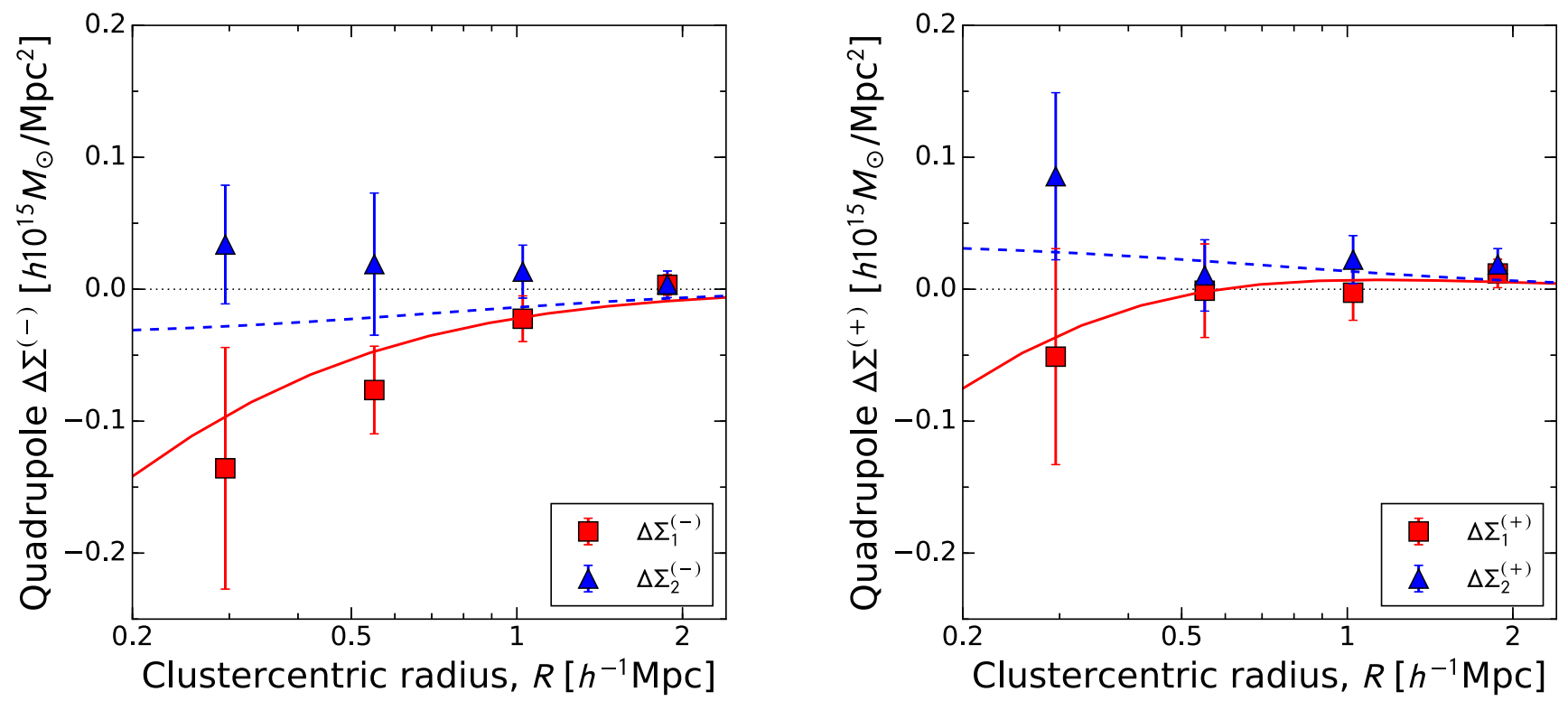

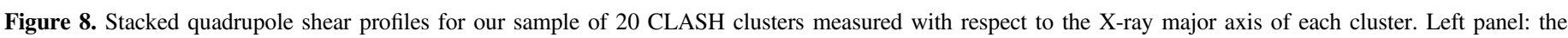

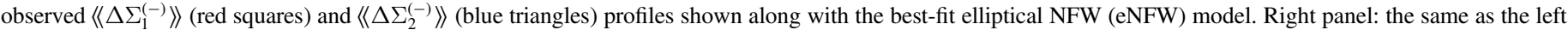
panel, but showing the results for the $\left.\left\langle\left\langle\Delta \Sigma_{1,2}^{(+)}\right)\right\rangle\right\rangle$profiles. The best-fit model was obtained from a simultaneous eNFW fit to the four quadrupole shear profiles.

\section{Summary}

In this paper, we have presented direct reconstructions of the 2D matter distribution in 20 high-mass clusters (Table 1) selected from the CLASH survey (Postman et al. 2012) by performing a joint weak-lensing analysis of $2 \mathrm{D}$ shear and azimuthally averaged magnification measurements. This complementary combination allows for a complete analysis of the field, effectively breaking the mass-sheet degeneracy.

We have simultaneously constrained the structure and morphology of each individual cluster by assuming an elliptical NFW halo. We have shown that spherical mass estimates of the clusters from azimuthally averaged weak-lensing measurements in previous work (Umetsu et al. 2014) are in excellent agreement with our results from the full 2D analysis (Figure 2). This indicates that systematic effects due to the azimuthal averaging applied to the weak-lensing data (Umetsu et al. 2014), as well as to the details of the inversion procedures (Section 3.6), are not significant in our mass determinations.

Combining all 20 clusters in our sample, we have measured the ellipticity of weak-lensing halos at the $5 \sigma$ significance level within a radial scale of $2 \mathrm{Mpc} h^{-1} \sim 1.1 r_{\text {vir }}$. The median projected axis ratio for the sample is constrained to be $\left\langle q_{\perp}\right\rangle=0.67 \pm 0.07$ (Section 5.2), which is in agreement with theoretical predictions of $\left\langle q_{\perp}\right\rangle=0.59-0.60$ from recent $N$ body simulations (Bonamigo et al. 2015; Suto et al. 2016) based on the standard collisionless $\Lambda \mathrm{CDM}$ model. However, we note that we expect the average axis ratio of the CLASH sample to be high due to a high fraction of relaxed clusters (Meneghetti et al. 2014). Hence, there could be a bias toward higher values of $q_{\perp}$ in our sample. A more quantitative comparison with theoretical expectations would thus require a detailed modeling of baryonic physics, accounting for the selection function.

We have studied the misalignment statistics of the BCG, $\mathrm{X}$-ray, SZE, and HST-lensing morphologies (Donahue et al. $2015,2016)$ with respect to our wide-field weak-lensing maps
(Section 5.3). Among the three baryonic tracers studied in this paper (i.e., BCGs, X-ray maps, and SZE maps), we find that the $\mathrm{X}$-ray morphology is best aligned with the weak-lensing mass distribution (Table 3 and Figure 6), with a median misalignment angle of $\langle|\Delta \mathrm{PA}|\rangle=21^{\circ} \pm 7^{\circ}$. This represents a $3.6 \sigma$ significance with respect to the null hypothesis of random orientations. Adopting the typical uncertainties in the PA measurements and correcting for the effect of noise bias, we constrain the intrinsic misalignment angle between the $\mathrm{X}$-ray and weak-lensing major axes to be $\langle|\Delta \mathrm{PA}|\rangle=16^{\circ} \pm 7^{\circ}$. A strong alignment is found between the weak-lensing and $H S T$ lensing major axes determined at different radial scales, with a median misalignment angle of $\langle|\Delta \mathrm{PA}|\rangle=16^{\circ} \pm 4^{\circ}$, corresponding to $7.7 \sigma$ significance. After applying the noise-bias correction, we find $\langle|\Delta \mathrm{PA}|\rangle=8^{\circ} \pm 4^{\circ}$. This strong alignment signal has been found despite using two independent data sets (HST versus ground-based observations) and substantially different modeling methods.

We also conducted a complementary stacked weak-lensing analysis of the 20 clusters using the quadrupole shear estimators of Clampitt \& Jain (2016). Assuming that the $\mathrm{X}$-ray brightness distribution is aligned with the projected mass distribution, we have obtained stacked constraints on the eNFW $q_{\perp}$ parameter of $0.67 \pm 0.10$ (Section 5.3.2), in excellent agreement with the results from our $2 \mathrm{D}$ weak-lensing analysis. This consistency supports the robustness of our results and suggests again a tight alignment between the intracluster gas and DM.

We note that while this paper was under review for publication, a paper by Okabe et al. (2018) appeared on the arXiv preprint service. They studied projected alignments of stellar, gas, and DM distributions in 40 cluster halos with $M_{200 \mathrm{c}}>5 \times 10^{13} M_{\odot}$ using cosmological hydrodynamical simulations. They showed that the total matter distribution is tightly aligned with the X-ray brightness distribution, with a level of misalignment that is consistent with our results, supporting our findings. 
Our observations support scenarios in which different cluster components, from the innermost region of BCGs to large intracluster scales of DM halos, share a similar orientation and formation history (West et al. 2017). Our results represent a first critical step in performing a non-spherical cluster analysis in combination with multiprobe data sets (Umetsu et al. 2015; Sereno et al. 2017b), an aim of the CLUMP-3D program. In our companion papers (Chiu et al. 2018; Sereno et al. 2018), we explicitly account for the effects of triaxiality in forwardmodeling our multiprobe data sets, simultaneously constraining the cluster mass, concentration, triaxial shape, and orientation from Bayesian inference. Extending this analysis with large, well-controlled samples of clusters from ongoing and planned surveys, such as the XXL survey (Pacaud et al. 2016; Pierre et al. 2016), the Subaru Hyper Suprime-Cam survey (Mandelbaum et al. 2018; Miyazaki et al. 2018a, 2018b; Oguri et al. 2018), the Dark Energy Survey, and the WFIRST and Euclid missions, will be a significant step forward in understanding the tidal and evolutionary effects of surrounding LSS on the intracluster mass distribution.

This work was made possible by the availability of highquality weak-lensing data produced by the CLASH survey. We are grateful to the CLASH team who enabled us to carry out this work. We thank the anonymous referee for constructive suggestions and comments. We thank Masamune Oguri for making his simulated Subaru Suprime-Cam observations available to us. K.U. acknowledges support from the Ministry of Science and Technology of Taiwan (grants MOST 103-2112-M-001-030-MY3 and MOST 106-2628-M-001-003MY3) and from the Academia Sinica Investigator Award. M.S. and S.E. acknowledge financial support from the contracts ASIINAF I/009/10/0, NARO15 ASI-INAF I/037/12/0, ASI 2015-046-R.0 and ASI-INAF n.2017-14-H.0. Support for D.G. was provided by NASA through Einstein Postdoctoral Fellowship grant number PF5-160138 awarded by the Chandra X-ray Center, which is operated by the Smithsonian Astrophysical Observatory for NASA under contract NAS8-03060. T.O. acknowledges support from the Ministry of Science and Technology of Taiwan under the grant MOST 106-2119-M001-031-MY3. M.M., M.S., S.E., and J.S. acknowledge support from the Italian Ministry of Foreign Affairs and International Cooperation, Directorate General for Country Promotion (Project "Crack the lens"). J.S. was supported by NSF/AST-1617022.

\section{Appendix A Shape Measurement Test}

We tested the reliability of our shape measurements of faint background galaxies by closely following the approach introduced by the STEP program (Heymans et al. 2006; Massey et al. 2007). To this end, we used a set of simulated sky images (Oguri et al. 2012) that closely match the characteristics of our Subaru/Suprime-Cam weak-lensing observations, especially in terms of the angular size of data and the range of signal-to-noise ratios of objects. The simulations optimized for our weak-lensing analysis allow us to have sufficient galaxies detected with high significance, $\nu_{g}>30$ (Section 3.3.2), with which to test our approach to the shear calibration. Besides, the simulations cover a wide range of input signal strengths up to $|g| \simeq 0.3$ as found in the inner

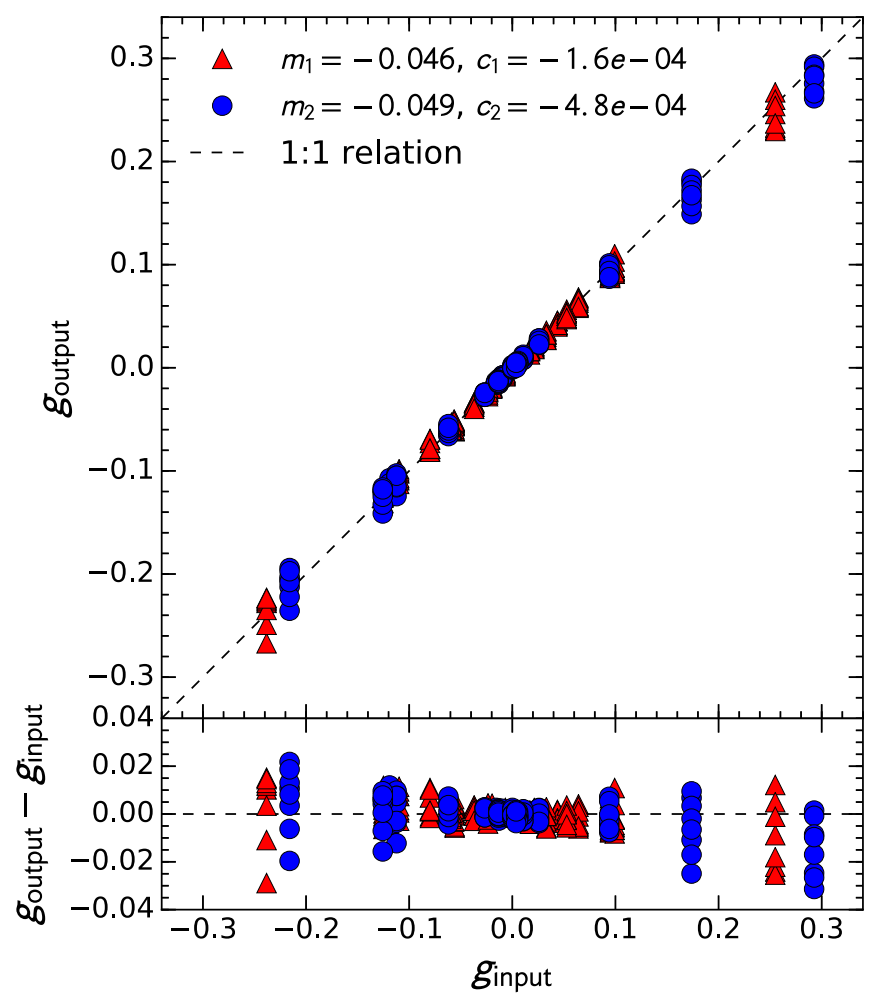

Figure 9. Results of the shear measurement test based on simulated Subaru/ Suprime-Cam images, showing the recovered shear signal $\left(g_{\text {output }}\right)$ as a function of the input signal $\left(g_{\text {input }}\right)$. Red triangles and blue circles show the results for $g_{1}$ and $g_{2}$, respectively. The lower panel shows the deviations from the input values, $g_{\text {output }}-g_{\text {input }}$. The dashed line shows the one-to-one relation.

regions of clusters, and thus are suitable for cluster weaklensing studies. The simulations assume constant shear across each simulated image, ignoring higher-order lensing effects that are present in the nonlinear regime close to the Einstein radius (Goldberg \& Bacon 2005; Okura et al. 2008; Schneider \& Er 2008), which is carefully avoided in our analysis. This simulation set has been used by several authors to test their analysis pipelines for cluster weak-lensing work based on Subaru/Suprime-Cam data (e.g., Umetsu et al. 2010; Oguri et al. 2012; Umetsu et al. 2012; Okabe et al. 2013; Okabe \& Smith 2016).

As described in Oguri et al. (2012; see also Okabe \& Smith 2016), a series of simulated images containing stars and sheared galaxies was created using the software packages STUFF (Bertin 2009) and GLAFIC (Oguri 2010). Each model galaxy is characterized by the sum of bulge and disk components, with Sérsic profile indices of $n=4$ and 1 , respectively. Galaxy images were convolved with an elliptical PSF model based on the Moffat profile $\Sigma(R) \propto\left[1+(R / a)^{2}\right]^{-\beta}$ (Oguri 2010), with seeing in the range 0 ". $5 \leqslant$ FWHM $\leqslant 1$ !" 1 and the Moffat power-law index $\beta$ in the range $3 \leqslant \beta \leqslant 12$. A large number of fits frames $\left(10 \mathrm{~K} \times 8 \mathrm{~K}\right.$ pixels with 0 ". 2 pixel $^{-1}$ sampling) matching the Suprime-Cam field of view were produced using the GLAFIC software. A total of 160 mock Suprime-Cam images were analyzed using the CLASH weaklensing analysis pipeline of Umetsu et al. (2014, Section 3.3.2). The results of the shear measurement test are summarized in Figures 9 and 10. Averaging over all of the analyzed images, we obtain a multiplicative shear calibration bias of 

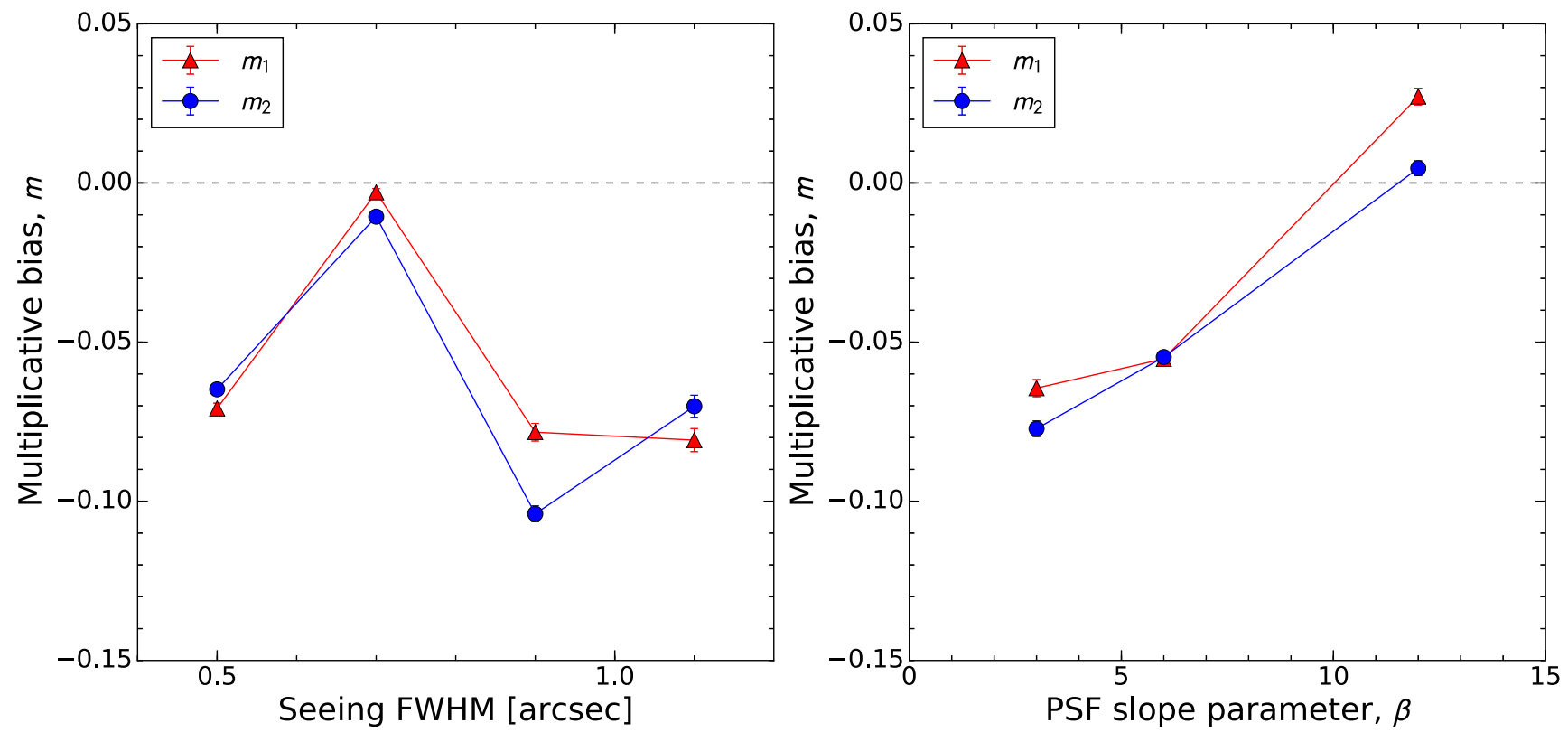

Figure 10. Multiplicative shear calibration bias $m_{\alpha}$ as a function of the seeing FWHM and the PSF outer slope parameter $\beta$ obtained using imaging simulations that match the characteristics of our Subaru/Suprime-Cam weak-lensing data. Red triangles and blue squares (with error bars) denote $m_{1}$ and $m_{2}$, respectively.

$m_{1}=-0.046$ and $m_{2}=-0.049$ and an additive bias of $c_{1}=-1.6 \times 10^{-4}$ and $c_{2}=-4.8 \times 10^{-4}$ (Figure 9; see Equation (14)). As shown in Figure 10, the degree of multiplicative bias $m_{\alpha}$ depends on the seeing conditions and the PSF properties to some degree, so that the variation with the PSF properties limits the shear calibration accuracy to $\delta m_{\alpha} \sim 0.05$.

\section{Appendix B \\ Response and Fisher Matrix}

In this appendix, we derive analytic expressions for the gradient and the Fisher matrix of the log-likelihood function for our joint shear and magnification weak-lensing analysis in the nonlinear subcritical regime of gravitational lensing. For simplicity of notation, we drop the subscripts $(\infty, g, \mu)$ (Section 2$)$ and simply use the symbols $(\kappa, \gamma)$ to denote the lensing fields. Note, in actual calculations, we account for the fact that the shear and magnification data have different depths, $\langle W\rangle_{g} \neq\langle W\rangle_{\mu}$. We also use the dimensionless convergence $\kappa=\Sigma_{\mathrm{c}}^{-1} \boldsymbol{\Sigma}$, instead of $\Sigma$, to denote our model vector, so that $\boldsymbol{\lambda}=(\boldsymbol{\kappa}, \boldsymbol{c})$. The expectation value of an observable quantity is denoted by a hat symbol.

In the subcritical nonlinear regime of lensing, the gradient of the log-posterior function $F(\boldsymbol{\lambda})$ (Section 3.5) with respect to $\kappa_{n}$ $\left(n=1,2, \ldots, N_{\text {pix }}\right)$ is expressed as

$$
\begin{aligned}
& \nabla_{n} F(\boldsymbol{\lambda})=\nabla_{n} l_{g}(\boldsymbol{\lambda})+\nabla_{n} l_{\mu}(\boldsymbol{\lambda}), \\
& \nabla_{n} l_{g}(\boldsymbol{\lambda})=\sum_{k, l=1}^{N_{\text {pix }}} \sum_{\alpha, \beta=1}^{2}\left(\mathcal{W}_{g}\right)_{\alpha \beta, k l} \mathcal{S}_{\alpha, k n}\left(\widehat{g}_{\beta}-g_{\beta}\right)_{l}, \\
& \nabla_{n} l_{\mu}(\boldsymbol{\lambda})=\sum_{i, j=1}^{N_{\text {bin }}}\left(\mathcal{W}_{\mu}\right)_{i j} \mathcal{R}_{i n}\left(\widehat{n}_{\mu}-n_{\mu}\right)_{j},
\end{aligned}
$$

where $\mathcal{S}_{\alpha, k n} \equiv \nabla_{n}\left(\widehat{g}_{\alpha, k}\right)$ and $R_{i n} \equiv \partial \widehat{n}_{\mu, i} / \partial \kappa_{n}$ are response matrices given by

$$
\begin{aligned}
\mathcal{S}_{\alpha, k n}= & \frac{1}{1-\kappa\left(\boldsymbol{\theta}_{k}\right)}\left[\mathcal{D}_{\alpha}\left(\boldsymbol{\theta}_{k}-\boldsymbol{\theta}_{n}\right)+\delta_{k n} \widehat{g}_{\alpha}\left(\boldsymbol{\theta}_{k}\right)\right], \\
\mathcal{R}_{i n}= & \sum_{k=1}^{N_{\text {pix }}} \mathcal{P}_{i k} \frac{\partial \widehat{n}_{\mu}\left(\boldsymbol{\theta}_{k}\right)}{\partial \kappa_{n}} \\
= & \left(5 s_{\text {eff }}-2\right) \sum_{k=1}^{N_{\text {pix }}} \mathcal{P}_{i k} \frac{\widehat{n}_{\mu}\left(\boldsymbol{\theta}_{k}\right)}{\Delta\left(\boldsymbol{\theta}_{k}\right)} \\
& \left(\left[1-\kappa\left(\boldsymbol{\theta}_{k}\right)\right] \delta_{k n}+\sum_{\alpha=1}^{2} \gamma_{\alpha}\left(\boldsymbol{\theta}_{k}\right) \mathcal{D}_{\alpha}\left(\boldsymbol{\theta}_{k}-\boldsymbol{\theta}_{n}\right)\right) .
\end{aligned}
$$

In the weak-lensing limit $(\kappa,|\gamma| \ll 1)$, the response matrices reduce to

$$
\begin{aligned}
\mathcal{S}_{\alpha, k n} & =\mathcal{D}_{\alpha}\left(\boldsymbol{\theta}_{k}-\boldsymbol{\theta}_{n}\right) \\
\mathcal{R}_{\text {in }} & =\left(5 s_{\mathrm{eff}}-2\right) \bar{n}_{\mu} \mathcal{P}_{i n} .
\end{aligned}
$$

We also calculate the derivatives of $F(\boldsymbol{\lambda})$ with respect to the calibration parameters $\boldsymbol{c}$.

Similarly, an analytic expression for the Fisher matrix $\mathcal{F}$ can be derived. In particular, the Fisher matrix elements $F_{m n}(m$, $\left.n=1,2, \ldots, N_{\text {pix }}\right)$ are given by

$$
\begin{aligned}
\mathcal{F}_{m n} & =\left\langle\frac{\partial^{2} l_{g}(\boldsymbol{\lambda})}{\partial \kappa_{m} \partial \kappa_{n}}\right\rangle\left|\hat{\boldsymbol{\lambda}}+\left\langle\frac{\partial^{2} l_{\mu}(\boldsymbol{\lambda})}{\partial \kappa_{m} \partial \kappa_{n}}\right\rangle\right|_{\widehat{\lambda}} \\
& =\sum_{k, l=1}^{N_{\mathrm{pix}}} \sum_{\alpha, \beta=1}^{2}\left(\mathcal{W}_{g}\right)_{\alpha \beta, k l} \mathcal{S}_{\alpha, k m} \mathcal{S}_{\beta, l n}+\sum_{i, j=1}^{N_{\text {bin }}}\left(\mathcal{W}_{\mu}\right)_{i j} \mathcal{R}_{i m} \mathcal{R}_{j n}
\end{aligned}
$$




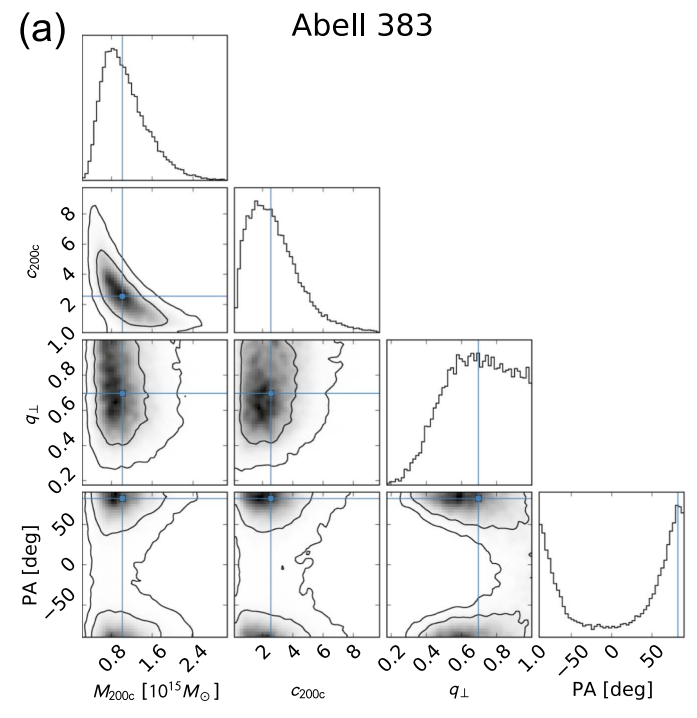

Abell 2261

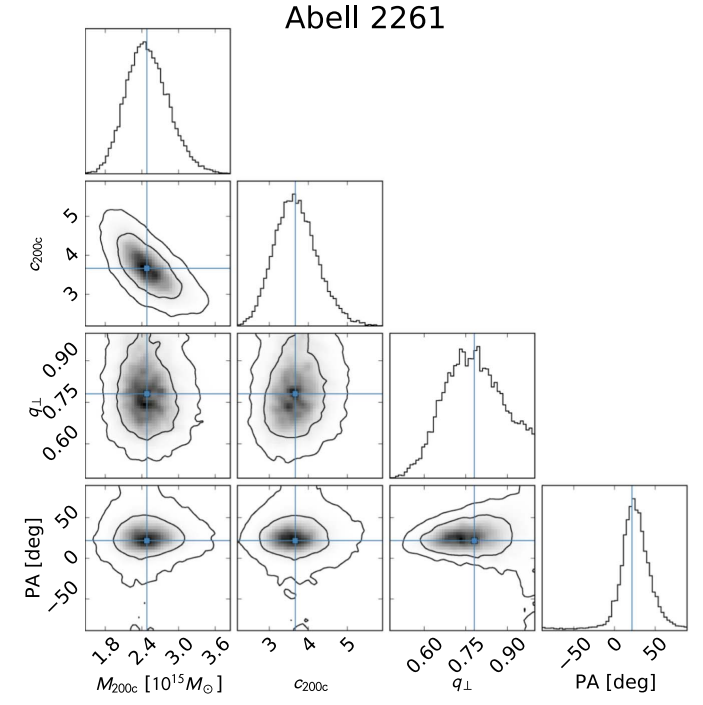

Abell 611

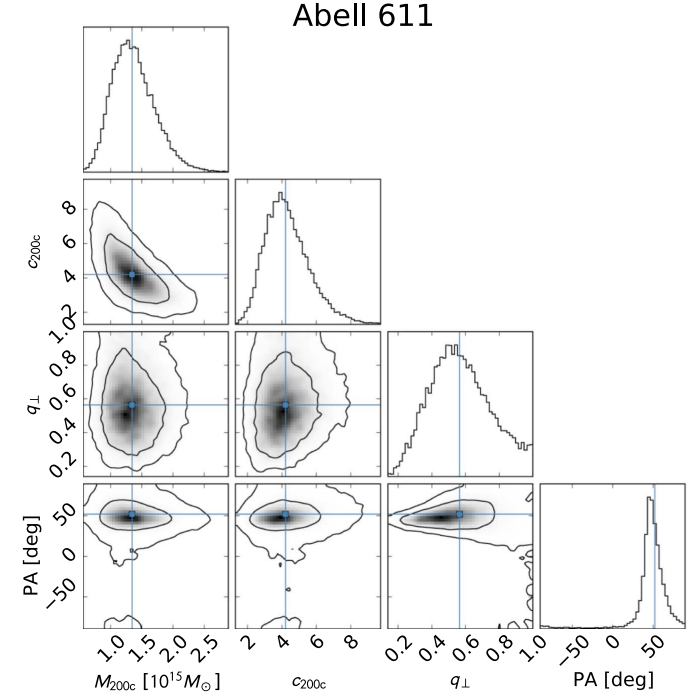

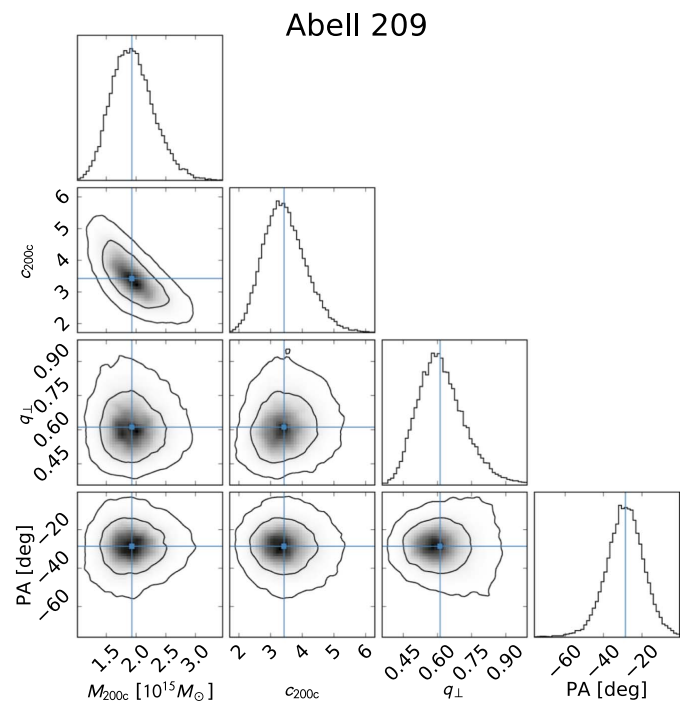
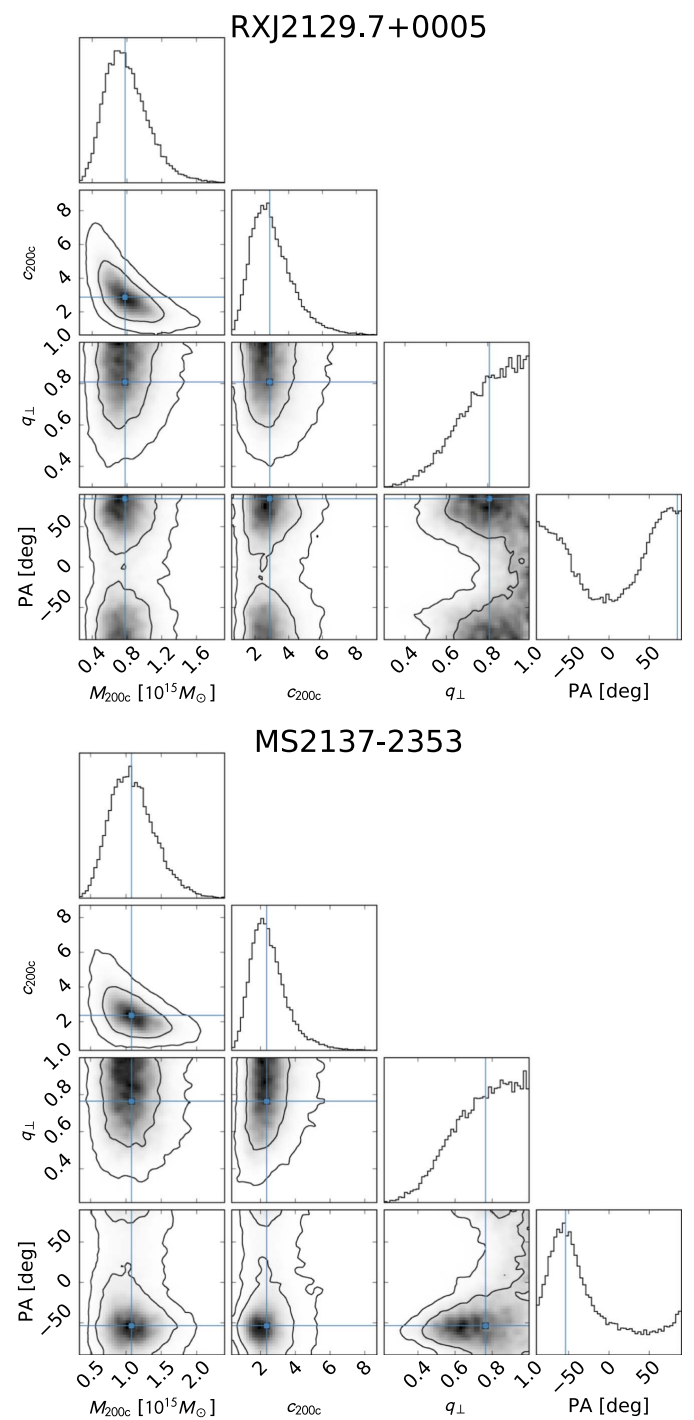

Figure 11. (a) Constraints on the eNFW model parameters $\left(M_{200 c}, c_{200 c}, q_{\perp}, \mathrm{PA}\right)$ for 20 individual CLASH clusters obtained using joint weak-lensing data sets of $2 \mathrm{D}$ gravitational shear and azimuthally averaged magnification measurements, showing marginalized 1D (histograms) and 2D (68\% and 95\% confidence level contour plots) posterior distributions. For each parameter, the blue solid line shows the biweight central location $\left(C_{\mathrm{BI}}\right)$ of the marginalized $1 \mathrm{D}$ distribution. (b) Posterior constraints on eNFW model parameters of CLASH clusters. (c) Posterior constraints on eNFW model parameters of CLASH clusters. (d) Posterior constraints on eNFW model parameters of CLASH clusters. For MACS J0647.7+7015, the marginalized 1D distribution of PA is bimodal, so that the biweight estimate of the center location $\left(C_{\mathrm{BI}}\right)$ lies between the two probability peaks. 

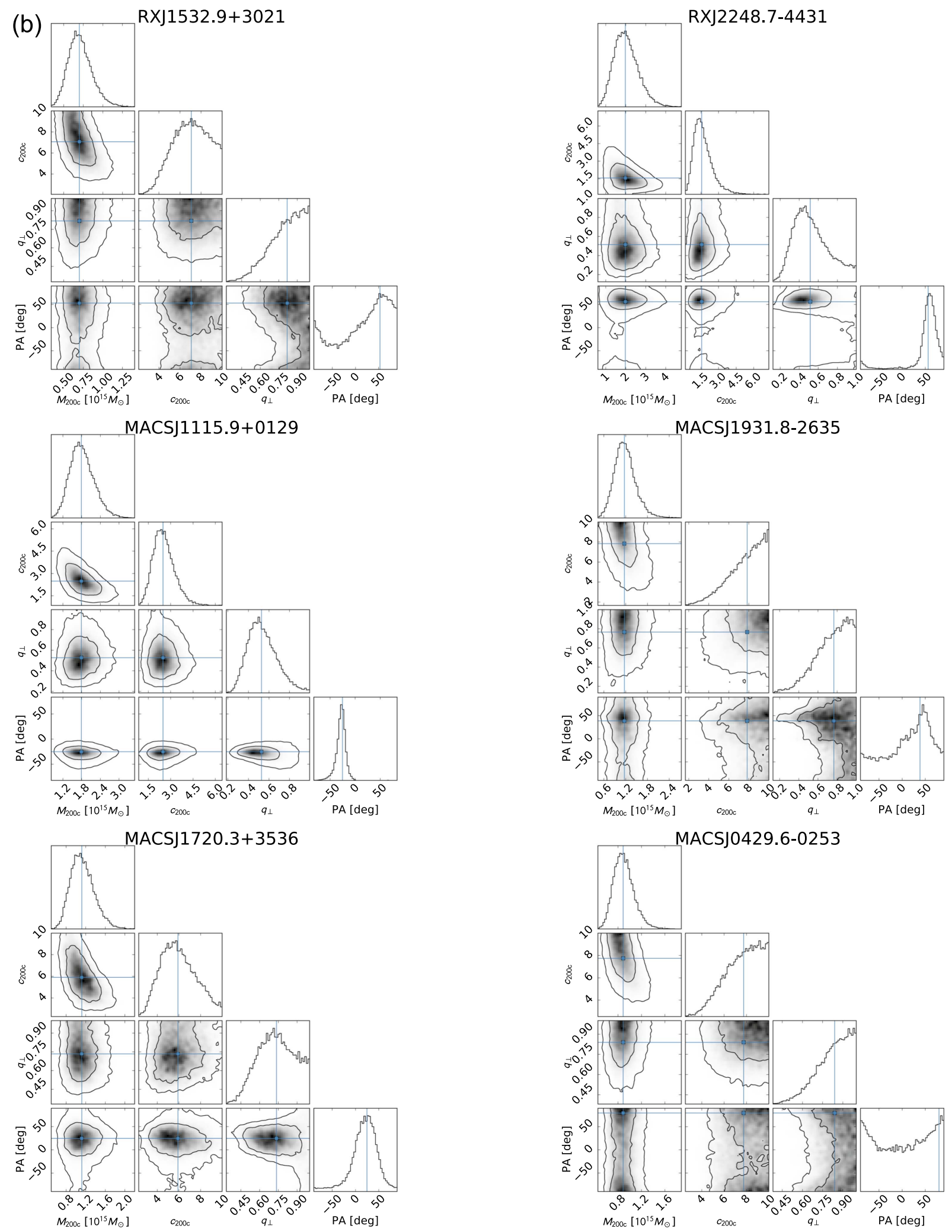

Figure 11. (Continued.) 

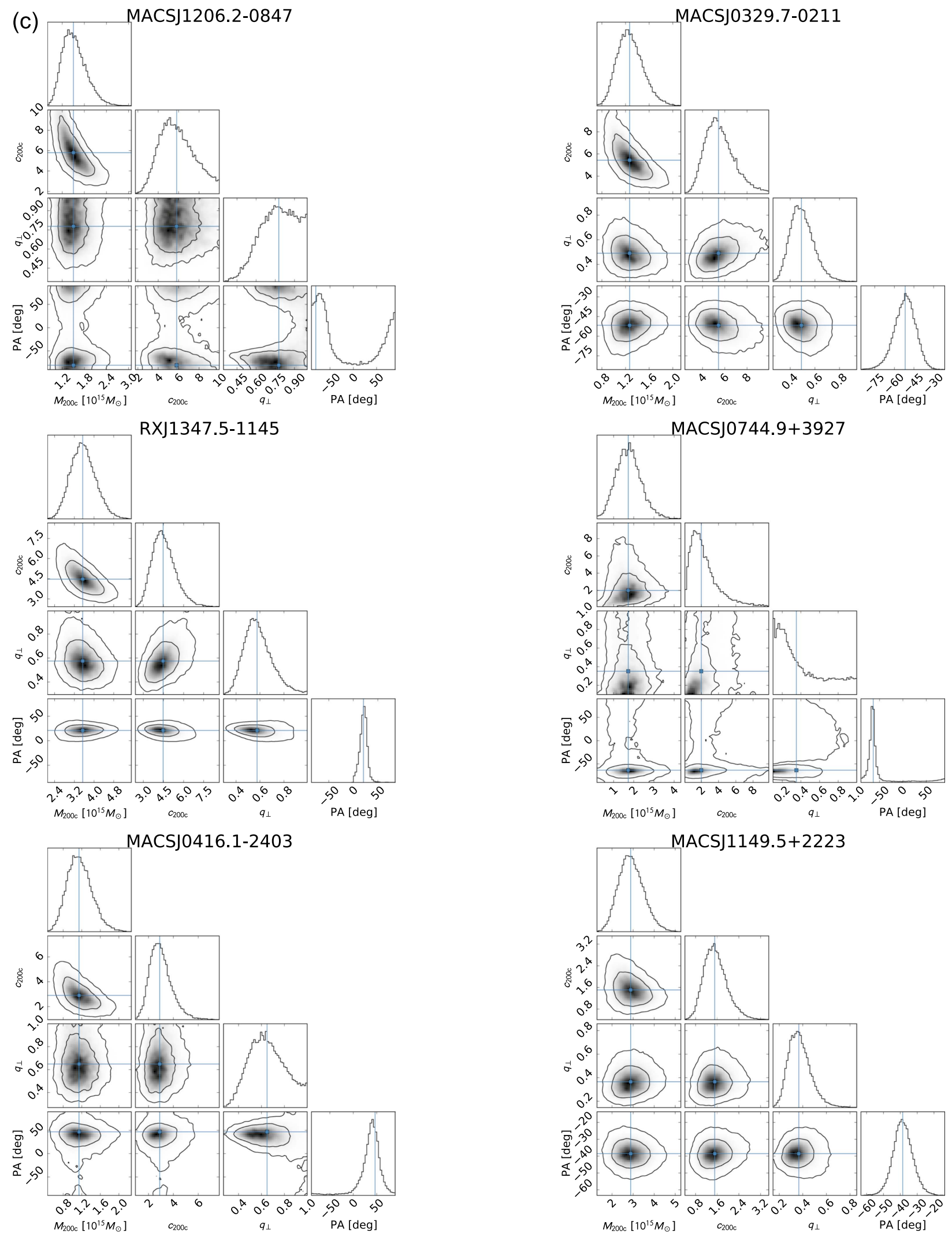

Figure 11. (Continued.) 

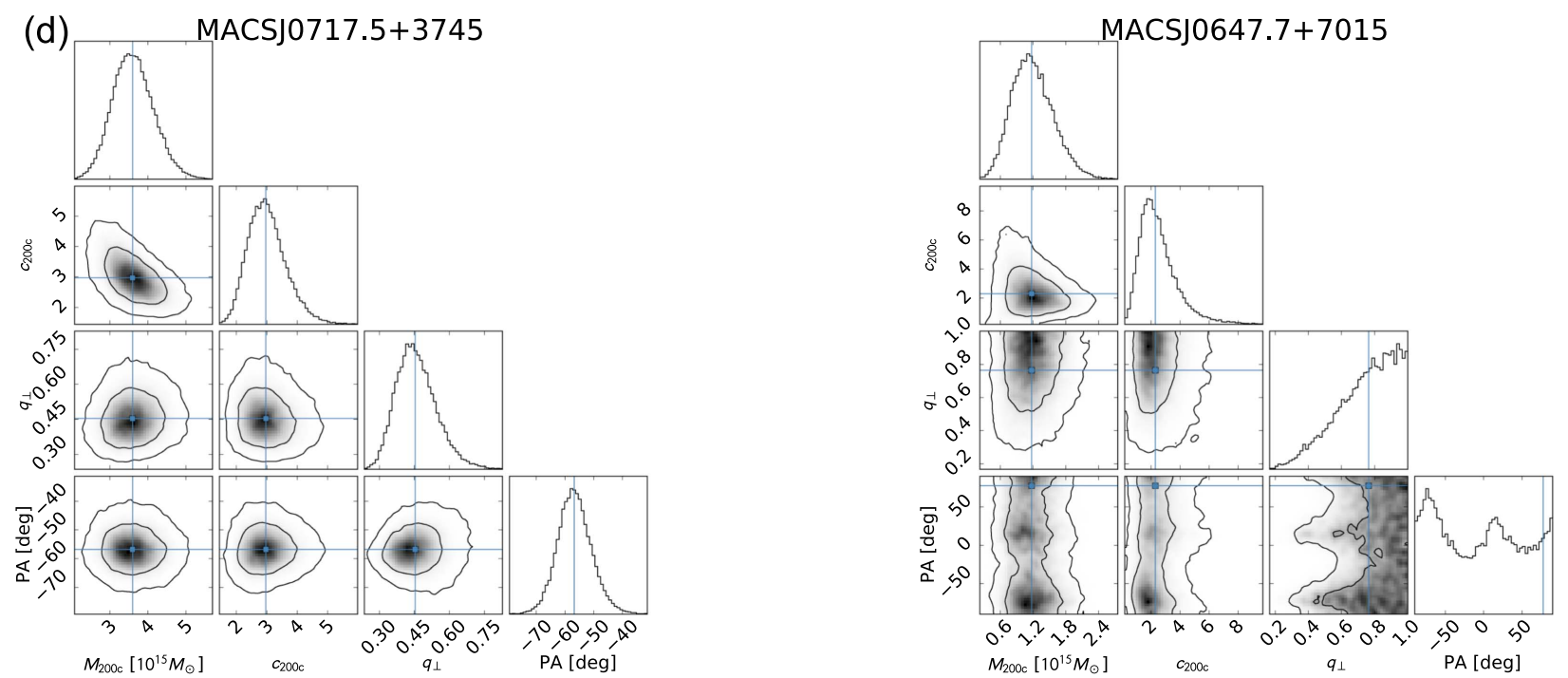

Figure 11. (Continued.)

\section{Appendix C \\ Marginalized Posterior Constraints on Elliptical NFW Parameters}

We show individual cluster constraints on the eNFW model parameters $\left(M_{200 c}, c_{200 c}, q_{\perp}, \mathrm{PA}\right)$ for our sample obtained from joint weak-lensing data sets of 2D gravitational shear and azimuthally averaged magnification measurements, showing marginalized $1 \mathrm{D}$ and $2 \mathrm{D}$ posterior distributions for each cluster. For each parameter, the blue solid line denotes the central location $\left(C_{\mathrm{BI}}\right)$ of the marginalized 1D distribution (see Table 1).

\section{ORCID iDs}

Keiichi Umetsu (1D https://orcid.org/0000-0002-7196-4822 Massimo Meneghetti (iD https://orcid.org/0000-00031225-7084

Adi Zitrin (iD https://orcid.org/0000-0002-0350-4488

Italo Balestra (iD) https://orcid.org/0000-0001-9660-894X

Jack Sayers (i) https://orcid.org/0000-0002-8213-3784

\section{References}

Adhikari, S., Chue, C. Y. R., \& Dalal, N. 2015, JCAP, 1, 009 Allen, S. W., Schmidt, R. W., Ebeling, H., Fabian, A. C., \& van Speybroeck, L. 2004, MNRAS, 353, 457

Annunziatella, M., Biviano, A., Mercurio, A., et al. 2014, A\&A, 571, A80

Bahcall, N. A., \& Fan, X. 1998, ApJ, 504, 1

Balestra, I., Mercurio, A., Sartoris, B., et al. 2016, ApJS, 224, 33

Bartelmann, M., \& Schneider, P. 2001, PhR, 340, 291

Becker, M. R., \& Kravtsov, A. V. 2011, ApJ, 740, 25

Beers, T. C., Flynn, K., \& Gebhardt, K. 1990, AJ, 100, 32

Benítez, N. 2000, ApJ, 536, 571

Benítez, N., Ford, H., Bouwens, R., et al. 2004, ApJS, 150, 1

Bertin, E. 2009, MmSAI, 80, 422

Bertin, E., \& Arnouts, S. 1996, A\&As, 117, 393

Bett, P., Eke, V., Frenk, C. S., et al. 2007, MNRAS, 376, 215

Bhattacharya, S., Habib, S., Heitmann, K., \& Vikhlinin, A. 2013, ApJ, 766, 32

Biviano, A., Rosati, P., Balestra, I., et al. 2013, A\&A, 558, A1

Bonamigo, M., Despali, G., Limousin, M., et al. 2015, MNRAS, 449, 3171

Bradač, M., Lombardi, M., \& Schneider, P. 2004, A\&A, 424, 13

Broadhurst, T. 1995, arXiv:astro-ph/9511150

Broadhurst, T., Benítez, N., Coe, D., et al. 2005a, ApJ, 621, 53

Broadhurst, T., Takada, M., Umetsu, K., et al. 2005b, ApJL, 619, L143

Broadhurst, T. J., Taylor, A. N., \& Peacock, J. A. 1995, ApJ, 438, 49
Cerny, C., Sharon, K., Andrade-Santos, F., et al. 2017, arXiv: 1710.09329 Chiu, I., Dietrich, J. P., Mohr, J., et al. 2016, MNRAS, 457, 3050 Chiu, I., Umetsu, K., Sereno, M., et al. 2018, arXiv:1804.00676 Cibirka, N., Cypriano, E. S., Brimioulle, F., et al. 2017, MNRAS, 468, 1092 Clampitt, J., \& Jain, B. 2016, MNRAS, 457, 4135

Coe, D., Umetsu, K., Zitrin, A., et al. 2012, ApJ, 757, 22

Colberg, J. M., White, S. D. M., Yoshida, N., et al. 2000, MNRAS, 319, 209

Colless, M., Dalton, G., Maddox, S., et al. 2001, MNRAS, 328, 1039

Coupon, J., Broadhurst, T., \& Umetsu, K. 2013, ApJ, 772, 65

Czakon, N. G., Sayers, J., Mantz, A., et al. 2015, ApJ, 806, 18

Despali, G., Giocoli, C., Angulo, R. E., et al. 2016, MNRAS, 456, 2486

Despali, G., Giocoli, C., \& Tormen, G. 2014, MNRAS, 443, 3208

Diego, J. M., Schmidt, K. B., Broadhurst, T., et al. 2018, MNRAS, 473, 4279

Diemer, B., \& Kravtsov, A. V. 2015, ApJ, 799, 108

Donahue, M., Connor, T., Fogarty, K., et al. 2015, ApJ, 805, 177

Donahue, M., Ettori, S., Rasia, E., et al. 2016, ApJ, 819, 36

Donahue, M., Voit, G. M., Mahdavi, A., et al. 2014, ApJ, 794, 136

Du, W., Fan, Z., Shan, H., et al. 2015, ApJ, 814, 120

Dutton, A. A., \& Macciò, A. V. 2014, MNRAS, 441, 3359

Evans, A. K. D., \& Bridle, S. 2009, ApJ, 695, 1446

Falco, E. E., Gorenstein, M. V., \& Shapiro, I. I. 1985, ApJL, 289, L1

Faltenbacher, A., Li, C., White, S. D. M., et al. 2009, RAA, 9, 41

Ford, J., Hildebrandt, H., Van Waerbeke, L., et al. 2012, ApJ, 754, 143

Garcia-Fernandez, M., Sánchez, E., Sevilla-Noarbe, I., et al. 2016, arXiv:1611. 10326

Geller, M. J., Diaferio, A., \& Kurtz, M. J. 2011, AJ, 142, 133

Goldberg, D. M., \& Bacon, D. J. 2005, ApJ, 619, 741

Gorenstein, M. V., Shapiro, I. I., \& Falco, E. E. 1988, ApJ, 327, 693

Grillo, C., Suyu, S. H., Rosati, P., et al. 2015, ApJ, 800, 38

Gruen, D., Brimioulle, F., Seitz, S., et al. 2013, MNRAS, 432, 1455

Gruen, D., Seitz, S., Becker, M. R., Friedrich, O., \& Mana, A. 2015, MNRAS, 449, 4264

Gruen, D., Seitz, S., Brimioulle, F., et al. 2014, MNRAS, 442, 1507

Hanami, H., Ishigaki, T., Fujishiro, N., et al. 2012, PASJ, 64, 70

Heymans, C., Van Waerbeke, L., Bacon, D., et al. 2006, MNRAS, 368, 1323 Hildebrandt, H. 2016, MNRAS, 455, 3943

Hildebrandt, H., Muzzin, A., Erben, T., et al. 2011, ApJL, 733, L30

Hjorth, J., \& Williams, L. L. R. 2010, ApJ, 722, 851

Hoekstra, H., Herbonnet, R., Muzzin, A., et al. 2015, MNRAS, 449, 685

Hu, W., \& White, M. 2001, ApJ, 554, 67

Huang, H.-J., Mandelbaum, R., Freeman, P. E., et al. 2016, MNRAS, 463, 222

Ilbert, O., Capak, P., Salvato, M., et al. 2009, ApJ, 690, 1236

Ilbert, O., Salvato, M., Le Floc'h, E., et al. 2010, ApJ, 709, 644

Jauzac, M., Eckert, D., Schaller, M., et al. 2017, arXiv:1711.01324

Jauzac, M., Richard, J., Jullo, E., et al. 2015, MNRAS, 452, 1437

Jing, Y. P., \& Suto, Y. 2000, ApJL, 529, L69

Jing, Y. P., \& Suto, Y. 2002, ApJ, 574, 538

Johnston, D. E., Sheldon, E. S., Tasitsiomi, A., et al. 2007, ApJ, 656, 27

Kaiser, N., \& Squires, G. 1993, ApJ, 404, 441

Kaiser, N., Squires, G., \& Broadhurst, T. 1995, ApJ, 449, 460 
Keeton, C. R. 2001, arXiv:astro-ph/0102341

Kitayama, T., Sasaki, S., \& Suto, Y. 1998, PASJ, 50, 1

Komatsu, E., Smith, K. M., Dunkley, J., et al. 2011, ApJS, 192, 18

Kotulla, R., Fritze, U., Weilbacher, P., \& Anders, P. 2009, MNRAS, 396, 462

Laigle, C., McCracken, H. J., Ilbert, O., et al. 2016, ApJS, 224, 24

Lee, J., \& Suto, Y. 2003, ApJ, 585, 151

Mandelbaum, R., Miyatake, H., Hamana, T., et al. 2018, PASJ, 70, S25

Mantz, A., Allen, S. W., Rapetti, D., \& Ebeling, H. 2010, MNRAS, 406, 1759

Massey, R., Heymans, C., Bergé, J., et al. 2007, MNRAS, 376, 13

Medezinski, E., Battaglia, N., Umetsu, K., et al. 2018, PASJ, 70, S28

Medezinski, E., Broadhurst, T., Umetsu, K., et al. 2010, MNRAS, 405, 257

Medezinski, E., Broadhurst, T., Umetsu, K., Benítez, N., \& Taylor, A. 2011, MNRAS, 414, 1840

Medezinski, E., Oguri, M., Nishizawa, A. J., et al. 2017, arXiv:1706.00427

Medezinski, E., Umetsu, K., Nonino, M., et al. 2013, ApJ, 777, 43

Medezinski, E., Umetsu, K., Okabe, N., et al. 2016, ApJ, 817, 24

Melchior, P., Gruen, D., McClintock, T., et al. 2017, MNRAS, 469, 4899

Meneghetti, M., Natarajan, P., Coe, D., et al. 2017, MNRAS, 472, 3177

Meneghetti, M., Rasia, E., Merten, J., et al. 2010, A\&A, 514, A93

Meneghetti, M., Rasia, E., Vega, J., et al. 2014, ApJ, 797, 34

Merten, J., Cacciato, M., Meneghetti, M., Mignone, C., \& Bartelmann, M. 2009, A\&A, 500, 681

Merten, J., Meneghetti, M., Postman, M., et al. 2015, ApJ, 806, 4

Miyazaki, S., Komiyama, Y., Kawanomoto, S., et al. 2018a, PASJ, 70, S1

Miyazaki, S., Komiyama, Y., Sekiguchi, M., et al. 2002, PASJ, 54, 833

Miyazaki, S., Oguri, M., Hamana, T., et al. 2018b, PASJ, 70, S27

Morandi, A., Limousin, M., Rephaeli, Y., et al. 2011, MNRAS, 416, 2567

Morandi, A., Limousin, M., Sayers, J., et al. 2012, MNRAS, 425, 2069

Morrison, C. B., Scranton, R., Ménard, B., et al. 2012, MNRAS, 426, 2489

Natarajan, P., \& Refregier, A. 2000, ApJL, 538, L113

Navarro, J. F., Frenk, C. S., \& White, S. D. M. 1996, ApJ, 462, 563

Navarro, J. F., Frenk, C. S., \& White, S. D. M. 1997, ApJ, 490, 493

Newman, A. B., Treu, T., Ellis, R. S., et al. 2013, ApJ, 765, 24

Niikura, H., Takada, M., Okabe, N., Martino, R., \& Takahashi, R. 2015, PASJ, 67, 103

Oguri, M. 2010, PASJ, 62, 1017

Oguri, M., Bayliss, M. B., Dahle, H., et al. 2012, MNRAS, 420, 3213

Oguri, M., \& Hamana, T. 2011, MNRAS, 414, 1851

Oguri, M., Lin, Y.-T., Lin, S.-C., et al. 2018, PASJ, 70, S20

Oguri, M., Takada, M., Okabe, N., \& Smith, G. P. 2010, MNRAS, 405, 2215

Oguri, M., Takada, M., Umetsu, K., \& Broadhurst, T. 2005, ApJ, 632, 841

Okabe, N., \& Smith, G. P. 2016, MNRAS, 461, 3794

Okabe, N., Smith, G. P., Umetsu, K., Takada, M., \& Futamase, T. 2013, ApJL, 769, L35

Okabe, T., Nishimichi, T., Oguri, M., et al. 2018, arXiv:1804.08843

Okumura, T., \& Jing, Y. P. 2009, ApJL, 694, L83

Okumura, T., Jing, Y. P., \& Li, C. 2009, ApJ, 694, 214

Okura, Y., Umetsu, K., \& Futamase, T. 2008, ApJ, 680, 1

Ostriker, J. P., \& Steinhardt, P. 2003, Sci, 300, 1909

Pacaud, F., Clerc, N., Giles, P. A., et al. 2016, A\&A, 592, A2

Park, C.-G., Ng, K.-W., Park, C., Liu, G.-C., \& Umetsu, K. 2003, ApJ, 589, 67

Penna-Lima, M., Bartlett, J. G., Rozo, E., et al. 2017, A\&A, 604, A89

Pierre, M., Pacaud, F., Adami, C., et al. 2016, A\&A, 592, A1

Postman, M., Coe, D., Benítez, N., et al. 2012, ApJS, 199, 25

Press, W. H., Teukolsky, S. A., Vetterling, W. T., \& Flannery, B. P. 1992 , Numerical Recipes in FORTRAN. The Art of Scientific Computing (2nd ed.; Cambridge: Cambridge Univ. Press)

Radovich, M., Formicola, I., Meneghetti, M., et al. 2015, A\&A, 579, A7
Rasia, E., Meneghetti, M., Martino, R., et al. 2012, NJPh, 14, 055018

Rosati, P., Balestra, I., Grillo, C., et al. 2014, Msngr, 158, 48

Rozo, E., \& Schmidt, F. 2010, arXiv:1009.5735

Sayers, J., Czakon, N. G., Mantz, A., et al. 2013, ApJ, 768, 177

Schive, H.-Y., Chiueh, T., \& Broadhurst, T. 2014, NatPh, 10, 496

Schneider, P., \& Er, X. 2008, A\&A, 485, 363

Schneider, P., King, L., \& Erben, T. 2000, A\&A, 353, 41

Schneider, P., \& Seitz, C. 1995, A\&A, 294, 411

Seitz, C., \& Schneider, P. 1997, A\&A, 318, 687

Seljak, U. 1998, ApJ, 506, 64

Sembolini, F., Yepes, G., De Petris, M., et al. 2013, MNRAS, 429, 323

Sereno, M., Covone, G., Izzo, L., et al. 2017a, MNRAS, 472, 1946

Sereno, M., Ettori, S., Meneghetti, M., et al. 2017b, MNRAS, 467, 3801

Sereno, M., Ettori, S., Umetsu, K., \& Baldi, A. 2013, MNRAS, 428, 2241

Sereno, M., Giocoli, C., Ettori, S., \& Moscardini, L. 2015, MNRAS, 449, 2024

Sereno, M., \& Umetsu, K. 2011, MNRAS, 416, 3187

Sereno, M., Umetsu, K., Ettori, S., et al. 2018, arXiv:1804.00667

Shaw, L. D., Weller, J., Ostriker, J. P., \& Bode, P. 2006, ApJ, 646, 815

Shin, T.-H., Clampitt, J., Jain, B., et al. 2018, MNRAS, 475, 2421

Smith, R. E., Peacock, J. A., Jenkins, A., et al. 2003, MNRAS, 341, 1311

Spergel, D. N., \& Steinhardt, P. J. 2000, PhRvL, 84, 3760

Stanford, S. A., Eisenhardt, P. R., \& Dickinson, M. 1998, ApJ, 492, 461

Stark, A. A. 1977, ApJ, 213, 368

Suto, D., Kitayama, T., Nishimichi, T., Sasaki, S., \& Suto, Y. 2016, PASJ, 68, 97

Suto, D., Peirani, S., Dubois, Y., et al. 2017, PASJ, 69, 14

Taylor, A. N., Dye, S., Broadhurst, T. J., Benitez, N., \& van Kampen, E. 1998, ApJ, 501, 539

Tegmark, M., Blanton, M. R., Strauss, M. A., et al. 2004, ApJ, 606, 702

Tudorica, A., Hildebrandt, H., Tewes, M., et al. 2017, arXiv:1710.06431

Umetsu, K. 2010, arXiv:1002.3952

Umetsu, K. 2013, ApJ, 769, 13

Umetsu, K., Birkinshaw, M., Liu, G.-C., et al. 2009, ApJ, 694, 1643

Umetsu, K., \& Broadhurst, T. 2008, ApJ, 684, 177

Umetsu, K., Broadhurst, T., Zitrin, A., et al. 2011a, ApJ, 738, 41

Umetsu, K., Broadhurst, T., Zitrin, A., Medezinski, E., \& Hsu, L. 2011b, ApJ, 729, 127

Umetsu, K., \& Diemer, B. 2017, ApJ, 836, 231

Umetsu, K., Medezinski, E., Broadhurst, T., et al. 2010, ApJ, 714, 1470

Umetsu, K., Medezinski, E., Nonino, M., et al. 2012, ApJ, 755, 56

Umetsu, K., Medezinski, E., Nonino, M., et al. 2014, ApJ, 795, 163

Umetsu, K., Sereno, M., Medezinski, E., et al. 2015, ApJ, 806, 207

Umetsu, K., Zitrin, A., Gruen, D., et al. 2016, ApJ, 821, 116

van Uitert, E., Hoekstra, H., Joachimi, B., et al. 2017, MNRAS, 467, 4131

Vega-Ferrero, J., Yepes, G., \& Gottlöber, S. 2017, MNRAS, 467, 3226

Vikhlinin, A., Kravtsov, A. V., Burenin, R. A., et al. 2009, ApJ, 692, 1060

von der Linden, A., Allen, M. T., Applegate, D. E., et al. 2014, MNRAS, 439, 2

West, M. J., de Propris, R., Bremer, M. N., \& Phillipps, S. 2017, NatAs, 1,0157

White, M., Carlstrom, J. E., Dragovan, M., \& Holzapfel, W. L. 1999, ApJ, 514,12

Williams, L. L. R., \& Hjorth, J. 2010, ApJ, 722, 856

Wong, K. C., Raney, C., Keeton, C. R., et al. 2017, ApJ, 844, 127

Wright, C. O., \& Brainerd, T. G. 2000, ApJ, 534, 34

Zhang, Y., Yang, X., Wang, H., et al. 2013, ApJ, 779, 160

Ziparo, F., Smith, G. P., Okabe, N., et al. 2016, MNRAS, 463, 4004

Zitrin, A., Fabris, A., Merten, J., et al. 2015, ApJ, 801, 44

Zitrin, A., Meneghetti, M., Umetsu, K., et al. 2013, ApJL, 762, L30 